Article

\title{
Investigating the Spectrum of Biological Activity of Substituted Quinoline-2-Carboxamides and Their Isosteres ${ }^{\dagger}$
}

Tomas Gonec ${ }^{1}$, Pavel Bobal ${ }^{1}$, Josef Sujan ${ }^{1}$, Matus Pesko ${ }^{2}$, Jiahui Guo ${ }^{3}$, Katarina Kralova ${ }^{4}$, Lenka Pavlacka ${ }^{1}$, Libor Vesely ${ }^{1}$, Eva Kreckova ${ }^{1}$, Jiri Kos ${ }^{1}$, Aidan Coffey ${ }^{3}$, Peter Kollar ${ }^{5}$, Ales Imramovsky ${ }^{6}$, Lukas Placek ${ }^{7}$ and Josef Jampilek ${ }^{1, *}$

1 Department of Chemical Drugs, Faculty of Pharmacy, University of Veterinary and Pharmaceutical Sciences, Palackeho 1/3, 61242 Brno, Czech Republic

2 Department of Ecosozology and Physiotactics, Faculty of Natural Sciences, Comenius University, Mlynska dolina Ch-2, 84215 Bratislava, Slovakia

3 Department of Biological Sciences, Cork Institute of Technology, Bishopstown, Cork, Ireland

4 Institute of Chemistry, Faculty of Natural Sciences, Comenius University, Mlynska dolina Ch-2, 84215 Bratislava, Slovakia

5 Department of Human Pharmacology and Toxicology, Faculty of Pharmacy, University of Veterinary and Pharmaceutical Sciences, Palackeho 1/3, 61242 Brno, Czech Republic

6 Institute of Organic Chemistry and Technology, Faculty of Chemical Technology, University of Pardubice, Studentska 573, 53210 Pardubice, Czech Republic

7 Pragolab s.r.o., Nad Krocinkou 55/285, 19000 Prague 9, Czech Republic

$\dagger$ Preliminary results were presented at The Fifteenth Electronic Conference on Synthetic Organic Chemistry (ECSOC-15, http://www.sciforum.net/presentation/588), 1-30 November 2011 (paper B585).

* Author to whom correspondence should be addressed; E-Mail: josef.jampilek@gmail.com; Tel.: +420-54-156-2925; Fax: +420-54-124-0607.

Received: 28 November 2011; in revised form: 27 December 2011 / Accepted: 4 January 2012 / Published: 10 January 2012

Abstract: In this study, a series of thirty-five substituted quinoline-2-carboxamides and thirty-three substituted naphthalene-2-carboxamides were prepared and characterized. They were tested for their activity related to the inhibition of photosynthetic electron transport (PET) in spinach (Spinacia oleracea L.) chloroplasts. Primary in vitro screening of the synthesized compounds was also performed against four mycobacterial species. $N$-Cycloheptylquinoline-2-carboxamide, $N$-cyclohexylquinoline-2-carboxamide and 
$N$-(2-phenylethyl)quinoline-2-carboxamide showed higher activity against $M$. tuberculosis than the standards isoniazid or pyrazinamide and 2-(pyrrolidin-1-ylcarbonyl)quinoline and 1-(2-naphthoyl)pyrrolidine expressed higher activity against M. kansasii and M. avium paratuberculosis than the standards isoniazid or pyrazinamide. The most effective antimycobacterial compounds demonstrated insignificant toxicity against the human monocytic leukemia THP-1 cell line. The PET-inhibiting activity expressed by $\mathrm{IC}_{50}$ value of the most active compound $N$-benzyl-2-naphthamide was $7.5 \mu \mathrm{mol} / \mathrm{L}$. For all compounds, the structure-activity relationships are discussed.

Keywords: quinolines; naphthalene; lipophilicity; photosynthetic electron transport inhibition; spinach chloroplasts; in vitro antimycobacterial activity; in vitro cytotoxicity

\section{Introduction}

The presence of an amide or thioamide (-NHCO- or -NHCS-) group is characteristic of a number of biologically active compounds, e.g., [1-9]. The wide spectrum of biological effects of substituted quinoline or naphthalene scaffolds includes especially antimicrobial, antineoplastics and antiviral activity [1,10-25]. In addition, some quinoline derivatives also showed noteworthy herbicidal activity $[13,15-18,26]$ and they were also found to be uncouplers of photosynthetic phosphorylation [27]. Over $50 \%$ of commercially available herbicides act by reversibly binding to photosystem II (PS II), a membrane-protein complex in the thylakoid membranes, which catalyses the oxidation of water and the reduction of plastoquinone [28], and thereby inhibit photosynthesis [29-31].

Both pharmaceuticals and pesticides are designed to target particular biological functions, and in some cases these functions overlap in their molecular target sites, or they target similar processes or molecules. Modern herbicides express low toxicity against mammals and one of the reasons is that mammals lack many of the target sites for herbicide action. At present, approximately 20 mechanisms of action of herbicides are known. It was determined that inhibitors of protoporphyrinogen oxidase, 4-hydroxyphenylpyruvate dioxygenase and glutamine synthetase inhibit these enzymes both in plants and mammals. However, the consequences of inhibition of the overlapping target site can be completely different for plants and animals. Therefore a compound that has lethal action on plants may be beneficial for mammals [32]. Such chemical compounds are characterized by low toxicity on mammals as a result of quick metabolism and/or elimination of herbicide from the mammal system. Taking into consideration that mammals may also have molecular sites of action of herbicides, most pharmaceutical companies until recently had pesticide divisions, sometimes with a different name. All compounds generated by either division of the company were evaluated for both pesticide and pharmaceutical uses. In the past, some leading pesticides have become pharmaceuticals and vice versa. However, little information of this type was published and must usually be deduced from patent literature. One of the exceptions is fluconazole, a fungicide product discovered by the pharmaceutical sector that is now used both as a pharmaceutical and patented as a crop production chemical [32-34].

In the context of the previously-described azanaphtalenes $[13,15-18,26]$ or various amides [3-9], new simple modifications of quinoline and naphtalene as quinoline isosteres that can 
trigger interesting biological activity were investigated. The compounds were tested for their photosynthesis-inhibiting activity-The inhibition of photosynthetic electron transport in spinach chloroplasts (Spinacia oleracea L.). The compounds were also assessed for activity against various mycobacterial species. Relationships between the structure and their in vitro antimycobacterial activities or/and activity related to inhibition of photosynthetic electron transport (PET) in spinach chloroplasts are discussed.

\section{Results and Discussion}

\subsection{Chemistry}

All the studied compounds were prepared according to Scheme 1. Condensation of the chlorides of 2-quinaldic and 2-naphthoic acids with commercially available substituted amines yielded a series of thirty-five substituted quinoline-2-carboxamides 1-19c and thirty-three substituted naphthalene-2-carboxamides 20-38c. Quinoline-2-carbonyl chloride was prepared using oxalyl chloride to ensure mild conditions and prevent quinoline nucleus chloration, whereas 2-naphtoyl chloride was obtained by the classical procedure using thionyl chloride.

Scheme 1. Synthesis of quinoline-2-carboxanilides 1-19c and naphthalene-2-carboxamides 20-38c.

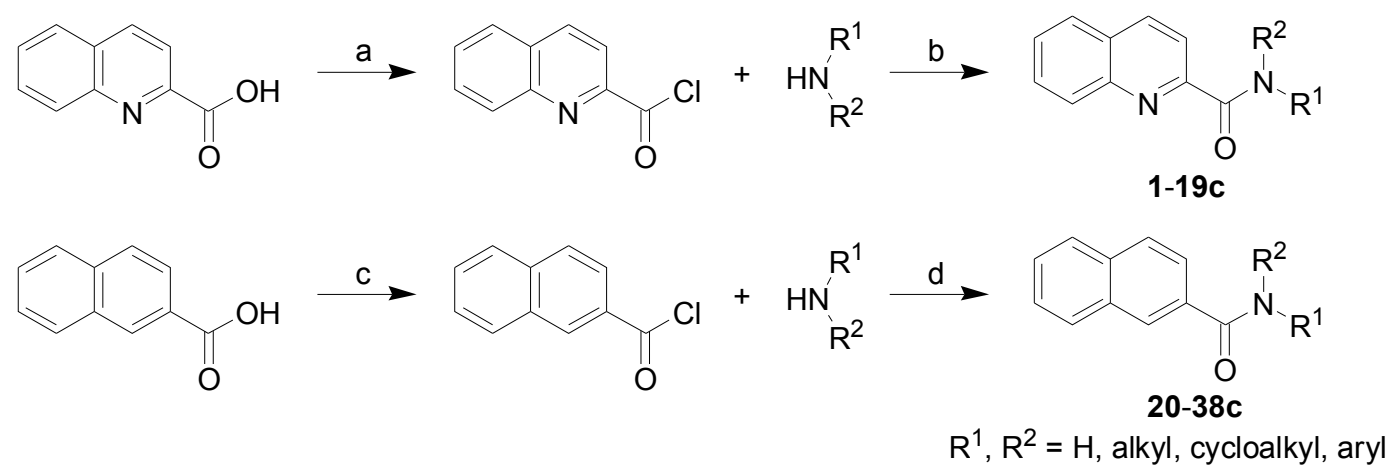

Reagents and conditions: (a) $(\mathrm{COCl})_{2}$, toluene; (b) TEA, toluene; (c) $\mathrm{SOCl}_{2}$, toluene; (d) TEA, $\mathrm{CH}_{2} \mathrm{Cl}_{2}$.

Hydrophobicities $(\log P$ ) of all compounds 1-38c were calculated using the commercially available program ACD/LogP (ACD/LogP ver. 1.0, Advanced Chemistry Development Inc., Toronto, Canada). The results are shown in Tables 1 and 2. Compounds show a wide range of lipophilicities, with $\log P$ (ACD/LogP) values from 1.15 (compound 3, pyrrolidinyl) to 6.98 (compound 2, octyl) within the series of quinolinecarboxamides and from 2.10 (compound 22, pyrrolidinyl) to 7.94 (compound 21, octyl) within the series of naphthalenecarboxamides. Individual substituents in the amide part of the discussed compounds also result in a wide range (from -0.39 to 1.26 ) of electronic properties expressed as $\sigma$ parameters [35-38]. 
Table 1. The calculated lipophilicities $(\log P / \mathrm{Clog} P$ ), electronic $\sigma$ parameters and $\mathrm{IC}_{50}[\mu \mathrm{mol} / \mathrm{L}]$ values related to PET inhibition in spinach chloroplasts of quinoline-2carboxamides 1-19c in comparison with 3-(3,4-dichlorophenyl)-1,1-dimethylurea (DCMU) standard. $\mathrm{NF}=$ not found in literature, $\mathrm{ND}=$ not determined due to precipitation during the experiment or interaction with 2,6-dichlorophenol-indophenol (DCIPP).

\begin{tabular}{|c|c|c|c|c|c|}
\hline & & & $N \prod_{O}^{\mathrm{R}^{1}} \mathrm{R}^{\mathrm{N}} \mathrm{R}^{1}$ & & \\
\hline Comp. & $\mathbf{R}^{1}$ & $\mathbf{R}^{2}$ & $\begin{array}{c}\text { PET inhibition } \\
\text { IC }_{50}[\mu \mathrm{mol} / \mathrm{L}]\end{array}$ & $\begin{array}{c}\log P \\
\mathrm{ACD} / \log \mathrm{P}\end{array}$ & $\begin{array}{c}\sigma \\
{[35]}\end{array}$ \\
\hline 1 & $i-\operatorname{Pr}$ & $\mathrm{H}$ & ND & $2.02 \pm 0.34$ & $-0.19[36]$ \\
\hline 2 & $-\mathrm{C}_{12} \mathrm{H}_{25}$ & $\mathrm{H}$ & 573 & $6.98 \pm 0.32$ & NF \\
\hline 3 & $-\left(\mathrm{CH}_{2}\right)_{4}-$ & & 1598 & $1.15 \pm 0.26$ & $\mathrm{NF}$ \\
\hline 4 & $-\left(\mathrm{CH}_{2}\right)_{5-}$ & & ND & $1.72 \pm 0.26$ & NF \\
\hline 5 & $c-\mathrm{Pn}$ & $\mathrm{H}$ & 762 & $2.63 \pm 0.33$ & $-0.20[36]$ \\
\hline 6 & $c-\mathrm{Hx}$ & $\mathrm{H}$ & 1041 & $3.20 \pm 0.33$ & $-0.15[36]$ \\
\hline 7 & $c-\mathrm{Hp}$ & $\mathrm{H}$ & ND & $3.76 \pm 0.33$ & NF \\
\hline 8 & $c-\mathrm{Oc}$ & $\mathrm{H}$ & 415 & $4.33 \pm 0.33$ & $\mathrm{NF}$ \\
\hline 9 & $\mathrm{Ph}$ & $\mathrm{H}$ & 85.1 & $2.90 \pm 0.34$ & $0.60[36] / 0$ \\
\hline 10 & $\mathrm{Bn}$ & $\mathrm{H}$ & 59.4 & $2.91 \pm 0.35$ & $0.22[36]$ \\
\hline 11 & $-\mathrm{C}_{2} \mathrm{H}_{4} \mathrm{Ph}$ & $\mathrm{H}$ & ND & $3.33 \pm 0.33$ & $0.08[36]$ \\
\hline $12 \mathrm{a}$ & 2-OH-Ph & $\mathrm{H}$ & 16.3 & $2.54 \pm 0.36$ & -0.09 \\
\hline $12 b$ & 3-OH-Ph & $\mathrm{H}$ & ND & $2.55 \pm 0.36$ & 0.12 \\
\hline $12 \mathrm{c}$ & 4-OH-Ph & $\mathrm{H}$ & ND & $2.15 \pm 0.35$ & -0.37 \\
\hline $13 \mathbf{a}$ & $2-\mathrm{OCH}_{3}-\mathrm{Ph}$ & $\mathrm{H}$ & ND & $2.80 \pm 0.36$ & -0.39 [37] \\
\hline $13 b$ & $3-\mathrm{OCH}_{3}-\mathrm{Ph}$ & $\mathrm{H}$ & ND & $3.06 \pm 0.36$ & 0.12 \\
\hline $13 \mathrm{c}$ & $4-\mathrm{OCH}_{3}-\mathrm{Ph}$ & $\mathrm{H}$ & ND & $2.85 \pm 0.36$ & -0.27 \\
\hline $14 a$ & $2-\mathrm{CH}_{3}-\mathrm{Ph}$ & $\mathrm{H}$ & 142.9 & $3.36 \pm 0.34$ & NF \\
\hline $14 \mathrm{~b}$ & $3-\mathrm{CH}_{3}-\mathrm{Ph}$ & $\mathrm{H}$ & 100.6 & $3.36 \pm 0.34$ & -0.07 \\
\hline $14 \mathrm{c}$ & $4-\mathrm{CH}_{3}-\mathrm{Ph}$ & $\mathrm{H}$ & $\mathrm{ND}$ & $3.36 \pm 0.34$ & -0.17 \\
\hline $15 a$ & 2-F-Ph & $\mathrm{H}$ & 98.1 & $2.86 \pm 0.44$ & $0.24[38]$ \\
\hline $15 b$ & 3-F-Ph & $\mathrm{H}$ & 86.9 & $3.38 \pm 0.44$ & 0.34 \\
\hline $15 \mathrm{c}$ & 4-F-Ph & $\mathrm{H}$ & 75.3 & $3.34 \pm 0.44$ & 0.06 \\
\hline $16 a$ & 2-Cl-Ph & $\mathrm{H}$ & 56.3 & $3.41 \pm 0.36$ & $0.20[38]$ \\
\hline $16 \mathrm{~b}$ & 3-Cl-Ph & $\mathrm{H}$ & 91.9 & $3.93 \pm 0.37$ & 0.37 \\
\hline $16 \mathrm{c}$ & 4-Cl-Ph & $\mathrm{H}$ & 147.6 & $3.89 \pm 0.36$ & 0.23 \\
\hline $17 \mathbf{a}$ & 2-Br-Ph & $\mathrm{H}$ & 92.2 & $3.58 \pm 0.44$ & $0.21[38]$ \\
\hline $17 \mathrm{~b}$ & $3-\mathrm{Br}-\mathrm{Ph}$ & $\mathrm{H}$ & 409.0 & $4.10 \pm 0.44$ & 0.39 \\
\hline $17 \mathrm{c}$ & 4-Br-Ph & $\mathrm{H}$ & 307.9 & $4.06 \pm 0.44$ & 0.23 \\
\hline $18 \mathrm{a}$ & $2-\mathrm{CF}_{3}-\mathrm{Ph}$ & $\mathrm{H}$ & 109.4 & $4.09 \pm 0.42$ & $\mathrm{NF}$ \\
\hline $18 \mathrm{~b}$ & $3-\mathrm{CF}_{3}-\mathrm{Ph}$ & $\mathrm{H}$ & 329.5 & $4.25 \pm 0.42$ & 0.43 \\
\hline $18 \mathrm{c}$ & $4-\mathrm{CF}_{3}-\mathrm{Ph}$ & $\mathrm{H}$ & ND & $3.91 \pm 0.41$ & 0.74 \\
\hline $19 a$ & $2-\mathrm{NO}_{2}-\mathrm{Ph}$ & $\mathrm{H}$ & ND & $3.15 \pm 0.38$ & 0.80 [38] \\
\hline $19 b$ & $3-\mathrm{NO}_{2}-\mathrm{Ph}$ & $\mathrm{H}$ & ND & $3.28 \pm 0.38$ & 0.71 \\
\hline $19 c$ & $4-\mathrm{NO}_{2}-\mathrm{Ph}$ & $\mathrm{H}$ & ND & $3.36 \pm 0.38$ & 1.26 \\
\hline DCMU & - & - & 1.9 & - & - \\
\hline
\end{tabular}


Table 2. The calculated lipophilicities $(\log P / \mathrm{Clog} P$ ), electronic $\sigma$ parameters and $\mathrm{IC}_{50}[\mu \mathrm{mol} / \mathrm{L}]$ values related to PET inhibition in spinach chloroplasts of naphthalene-2carboxamides 20-38c in comparison with 3-(3,4-dichlorophenyl)-1,1-dimethylurea (DCMU) standard. $\mathrm{NF}=$ not found in literature, $\mathrm{ND}=$ not determined due to precipitation during the experiment or interaction with 2,6-dichlorophenol-indophenol (DCIPP).

\begin{tabular}{|c|c|c|c|c|c|}
\hline & & & $\prod_{O}^{R^{\prime}} R^{1}$ & & \\
\hline Comp. & $\mathbf{R}^{1}$ & $\mathbf{R}^{2}$ & $\begin{array}{c}\text { PET inhibition } \\
\text { IC }_{50}[\mu \mathrm{mol} / \mathrm{L}]\end{array}$ & $\begin{array}{c}\log P \\
\mathrm{ACD} / \log P \\
\end{array}$ & $\begin{array}{c}\sigma \\
{[35]}\end{array}$ \\
\hline 20 & $i-\operatorname{Pr}$ & $\mathrm{H}$ & 353 & $2.97 \pm 0.28$ & $-0.19[36]$ \\
\hline 21 & $-\mathrm{C}_{12} \mathrm{H}_{25}$ & $\mathrm{H}$ & 845 & $7.94 \pm 0.28$ & NF \\
\hline 22 & $-\left(\mathrm{CH}_{2}\right)_{4}-$ & & ND & $2.10 \pm 0.23$ & NF \\
\hline 23 & $-\left(\mathrm{CH}_{2}\right)_{5-}^{-}$ & & ND & $2.67 \pm 0.23$ & NF \\
\hline 24 & $c$-Pn & $\mathrm{H}$ & ND & $3.59 \pm 0.28$ & $-0.20[36]$ \\
\hline 25 & $c-\mathrm{Hx}$ & $\mathrm{H}$ & ND & $4.15 \pm 0.28$ & $-0.15[36]$ \\
\hline 26 & $c-\mathrm{Hp}$ & $\mathrm{H}$ & 216 & $4.21 \pm 0.28$ & NF \\
\hline 27 & $c-\mathrm{Oc}$ & $\mathrm{H}$ & 688 & $5.28 \pm 0.28$ & NF \\
\hline 28 & $\mathrm{Ph}$ & $\mathrm{H}$ & 20.7 & $3.85 \pm 0.29$ & $0.60[36] / 0$ \\
\hline 29 & $\mathrm{Bn}$ & $\mathrm{H}$ & 7.5 & $3.87 \pm 0.28$ & $0.22[36]$ \\
\hline 30 & $-\mathrm{C}_{2} \mathrm{H}_{4} \mathrm{Ph}$ & $\mathrm{H}$ & ND & $4.28 \pm 0.29$ & $0.08[36]$ \\
\hline 31b & 3-OH-Ph & $\mathrm{H}$ & ND & $3.50 \pm 0.31$ & 0.12 \\
\hline $31 \mathrm{c}$ & 4-OH-Ph & $\mathrm{H}$ & ND & $3.11 \pm 0.30$ & -0.37 \\
\hline $32 a$ & $2-\mathrm{OCH}_{3}-\mathrm{Ph}$ & $\mathrm{H}$ & 763.0 & $3.75 \pm 0.32$ & $-0.39[37]$ \\
\hline 32b & $3-\mathrm{OCH}_{3}-\mathrm{Ph}$ & $\mathrm{H}$ & 306.2 & $4.01 \pm 0.32$ & 0.12 \\
\hline $32 \mathrm{c}$ & $4-\mathrm{OCH}_{3}-\mathrm{Ph}$ & $\mathrm{H}$ & ND & $3.80 \pm 0.32$ & -0.27 \\
\hline $33 a$ & $2-\mathrm{CH}_{3}-\mathrm{Ph}$ & $\mathrm{H}$ & ND & $4.31 \pm 0.30$ & NF \\
\hline 33b & $3-\mathrm{CH}_{3}-\mathrm{Ph}$ & $\mathrm{H}$ & ND & $4.31 \pm 0.30$ & -0.07 \\
\hline $33 \mathrm{c}$ & $4-\mathrm{CH}_{3}-\mathrm{Ph}$ & $\mathrm{H}$ & ND & $4.31 \pm 0.30$ & -0.17 \\
\hline $34 a$ & 2-F-Ph & $\mathrm{H}$ & ND & $3.82 \pm 0.40$ & $0.24[38]$ \\
\hline 34b & 3-F-Ph & $\mathrm{H}$ & ND & $4.34 \pm 0.41$ & 0.34 \\
\hline $34 c$ & 4-F-Ph & $\mathrm{H}$ & ND & $4.30 \pm 0.41$ & 0.06 \\
\hline $35 a$ & 2-Cl-Ph & $\mathrm{H}$ & ND & $4.36 \pm 0.32$ & $0.20[38]$ \\
\hline $35 b$ & 3-Cl-Ph & $\mathrm{H}$ & 81.0 & $4.88 \pm 0.33$ & 0.37 \\
\hline $35 \mathrm{c}$ & 4-Cl-Ph & $\mathrm{H}$ & ND & $4.84 \pm 0.32$ & 0.23 \\
\hline $36 a$ & 2-Br-Ph & $\mathrm{H}$ & ND & $4.54 \pm 0.40$ & $0.21[38]$ \\
\hline $36 \mathrm{~b}$ & $3-\mathrm{Br}-\mathrm{Ph}$ & $\mathrm{H}$ & 102.8 & $5.06 \pm 0.41$ & 0.39 \\
\hline $36 c$ & 4-Br-Ph & $\mathrm{H}$ & ND & $5.02 \pm 0.41$ & 0.23 \\
\hline $37 \mathrm{~b}$ & $3-\mathrm{CF}_{3}-\mathrm{Ph}$ & $\mathrm{H}$ & 309.4 & $5.20 \pm 0.39$ & 0.43 \\
\hline $37 \mathrm{c}$ & $4-\mathrm{CF}_{3}-\mathrm{Ph}$ & $\mathrm{H}$ & ND & $4.86 \pm 0.37$ & 0.74 \\
\hline $38 \mathrm{a}$ & $2-\mathrm{NO}_{2}-\mathrm{Ph}$ & $\mathrm{H}$ & ND & $4.10 \pm 0.34$ & $0.80[38]$ \\
\hline $38 \mathrm{~b}$ & $3-\mathrm{NO}_{2}-\mathrm{Ph}$ & $\mathrm{H}$ & 633.3 & $4.24 \pm 0.34$ & 0.71 \\
\hline $38 c$ & $4-\mathrm{NO}_{2}-\mathrm{Ph}$ & $\mathrm{H}$ & 260.0 & $4.31 \pm 0.34$ & 1.26 \\
\hline DCMU & - & - & 1.9 & - & - \\
\hline
\end{tabular}




\subsection{Biological Activities}

The compounds under investigation could be divided into two groups based on their chemical structure: Group 1 included quinoline-2-carboxamides 1-19c; Group 2 contained naphthalene-2-carboxamides 20-38c. Compounds within both series can be also divided according to whether they contain an aromatic or a non-aromatic amine. The compounds showed a wide range of biological activities and some interesting structure-activity relationships were observed. All the results are summarized in Tables 1-3. Generally, all the discussed compounds exhibited problematic solubility, but quinoline derivatives generally possess better aqueous solubility in comparison to the naphthamides.

Table 3. In vitro antimycobacterial activity $\left(\mathrm{IC}_{90}\right)$ of compounds 1-3, 5-7, 11, 14b, 22 and 32a in comparison with standards isoniazid (INH) and pyrazinamide (PZA), in vitro cytotoxicity assay $\left(\mathrm{LD}_{50}\right)$ of compounds $3,7,11$ and 22 and calculated selectivity index (SI). ND = not determined; used $\mathrm{IC}_{90}$ for calculation of SI is marked by *.

\begin{tabular}{|c|c|c|c|c|c|c|}
\hline \multirow[b]{2}{*}{ Comp. } & \multicolumn{4}{|c|}{$\mathrm{MIC} / \mathrm{IC}_{90}[\mu \mathrm{mol} / \mathrm{L}]$} & \multirow{2}{*}{$\begin{array}{c}\mathbf{L D}_{\mathbf{5 0}} \\
{[\boldsymbol{\mu \mathrm { mol }} / \mathbf{L}]}\end{array}$} & \multirow{2}{*}{$\begin{array}{c}\mathrm{SI} \\
{\left[\mathrm{LD}_{\mathbf{5 0}} / \mathrm{IC}_{\mathbf{9 0}}\right]}\end{array}$} \\
\hline & M. tuberculosis \# & $\begin{array}{l}\text { M. avium } \\
\text { complex }\end{array}$ & $\begin{array}{c}\text { M. avium } \\
\text { paratuberculosis }\end{array}$ & M. kansasii & & \\
\hline 1 & 280 & 583 & 1167 & 1167 & ND & - \\
\hline 2 & 367 & 367 & 734 & 734 & $\mathrm{ND}$ & - \\
\hline 3 & 552 & 552 & $111 *$ & $111 *$ & $>100$ & $>0.90$ \\
\hline 5 & 491 & 491 & 491 & 491 & ND & - \\
\hline 6 & 125 & 520 & 520 & 520 & ND & - \\
\hline 7 & $111 *$ & 466 & 223 & 466 & $62 \pm 4.5$ & 0.56 \\
\hline 11 & $109 *$ & 452 & 905 & 452 & $>100$ & $>0.92$ \\
\hline $14 \mathrm{~b}$ & 469 & 469 & 939 & 469 & ND & - \\
\hline 22 & 554 & 554 & $111 *$ & $111 *$ & $>100$ & $>0.90$ \\
\hline $32 \mathbf{a}$ & 901 & 451 & 216 & 451 & ND & - \\
\hline INH & $>729$ & $<72.9$ & $>729$ & $<72.9$ & ND & - \\
\hline PZA & $>812$ & $>812$ & $>812$ & $>812$ & ND & - \\
\hline
\end{tabular}

${ }^{\#}$ Clinical isolate of M. tuberculosis CUH071 (Cork University Hospital TB lab), with partial INH and PZA resistance.

\subsubsection{Inhibition of Photosynthetic Electron Transport (PET) in Spinach Chloroplasts}

The activity of all the evaluated derivatives related to inhibition of photosynthetic electron transport (PET) in spinach (Spinacia oleracea L.) chloroplasts was moderate or rather low relative to the standard, see Tables 1 and 2. $N$-(2-Hydroxyphenyl)quinoline-2-carboxamide (12a) expressed the highest PET-inhibiting activity $\left(\mathrm{IC}_{50}=16.3 \mu \mathrm{mol} / \mathrm{L}\right)$ within the series of quinolinecarboxamides whereas $N$-benzyl-2-naphthamide (29) from the second set investigated was two-fold more effective with a PET inhibition $\mathrm{IC}_{50}$ value of $7.5 \mu \mathrm{mol} / \mathrm{L}$. The PET-inhibiting activity was expressed by the negative logarithm of the $\mathrm{IC}_{50}$ value (compound concentration in mol/L causing $50 \%$ inhibition of PET). Despite the relatively low inhibitory activity of the rest studied compounds, correlations between $\log \left(1 / \mathrm{IC}_{50}\right)$ and the lipophilicity expressed as $\log P(\mathrm{ACD} / \mathrm{Log} \mathrm{P})$ or electronic properties expressed as Hammett's $\sigma$ parameters of the individual substituents in compounds $\mathbf{1}-\mathbf{3 8 c}$ were 
performed, see Figures 1, 2 and 3. Generally, non-aromatic $N$-substituents showed lower PET-inhibiting activity than anilides.

The PET-inhibiting activity of the evaluated quinoline derivatives (Group 1) is summarized in Table 1. According to both Figure 1, where all compounds except substituted phenyl rings are illustrated, it can be stated that the dependence of PET-inhibiting activity on the lipophilicity showed a parabolic course. Cyclic non-aromatic $N$-substituents are illustrated in Figure 1a, where the most active compound $N$-cyclooctyl 8 has a lipophilicity optimum at $\log P=4.33$. The dependence of PET-inhibiting activity on electronic constants $\sigma$ of non-aromatic as well as phenyl $\mathbf{9}$ and benzyl $\mathbf{1 0}$ substituents obtained from literature [36] seems to also follow a parabolic course, see Figure $1 b$. Benzyl derivative $\mathbf{1 0}$ showed an optimum of weak electron-withdrawing effect influencing the electronic density of the amido moiety. It is evident that bulkiness of the $N$-substituents did not influence PET-inhibiting activity.

Figure 1. Relationships between PET inhibition $\log \left(1 / \mathrm{IC}_{50}\right)[\mathrm{mol} / \mathrm{L}]$ in spinach chloroplasts and lipophilicity (a) or $N$-substituent electronic $\sigma$ parameters (b) of studied compounds 1-10.

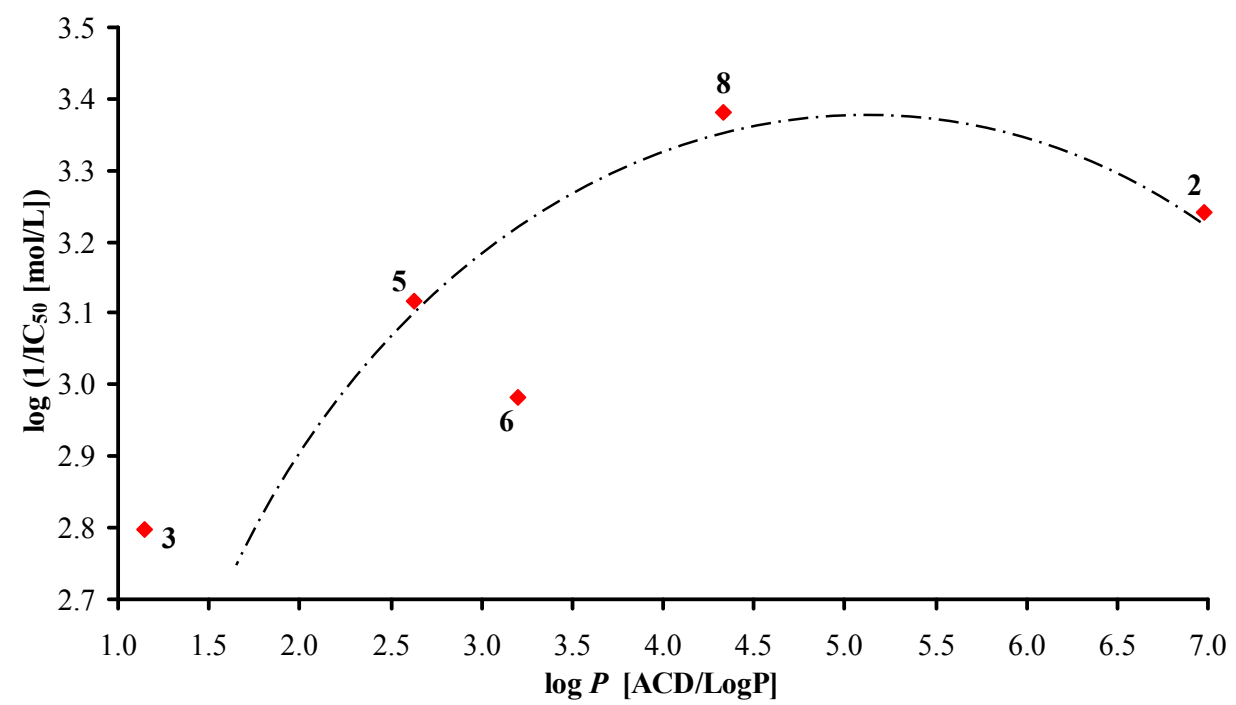

(a)

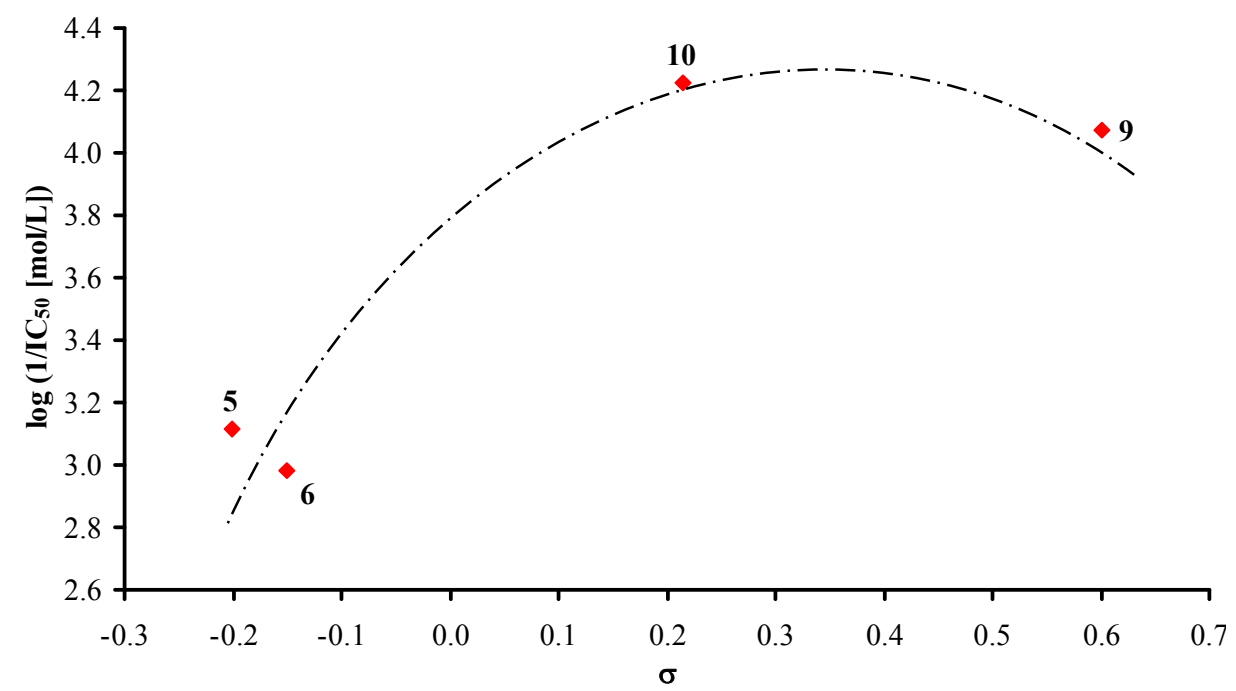

(b) 
Figure 2 shows the correlations between $\log \left(1 / \mathrm{IC}_{50}\right)$ and the lipophilicity or Hammett's $\sigma$ parameters of the individual anilide substituents in compounds 9, 12a-19c. Based on the results obtained it is not possible to decide whether some of the ortho-, meta- or para-positions are preferred from the PET-inhibiting activity point of view. Nevertheless, according to Figure 2a it can be stated that the PET inhibition showed linear decrease with increasing lipophilicity and the lipophilicity of the compounds was decisive for PET inhibition:

$$
\begin{gathered}
\log \left(1 / \mathrm{IC}_{50}\right)=5.952( \pm 0.406)-0.559( \pm 0.113) \log P \\
\mathrm{r}=0.819, \mathrm{~s}=0.211, \mathrm{~F}=24.50, \mathrm{n}=14
\end{gathered}
$$

On the other hand, the biological activity was also affected by electronic $\sigma$ properties of these anilide substituents, see Figure $2 b$.

Figure 2. Relationships between PET inhibition $\log \left(1 / \mathrm{IC}_{50}\right)[\mathrm{mol} / \mathrm{L}]$ in spinach chloroplasts and lipophilicity (a) or $N$-substituent electronic Hammett's $\sigma$ parameters (b) of studied compounds $\mathbf{9}, \mathbf{1 2 a}-\mathbf{1 9 c}$.

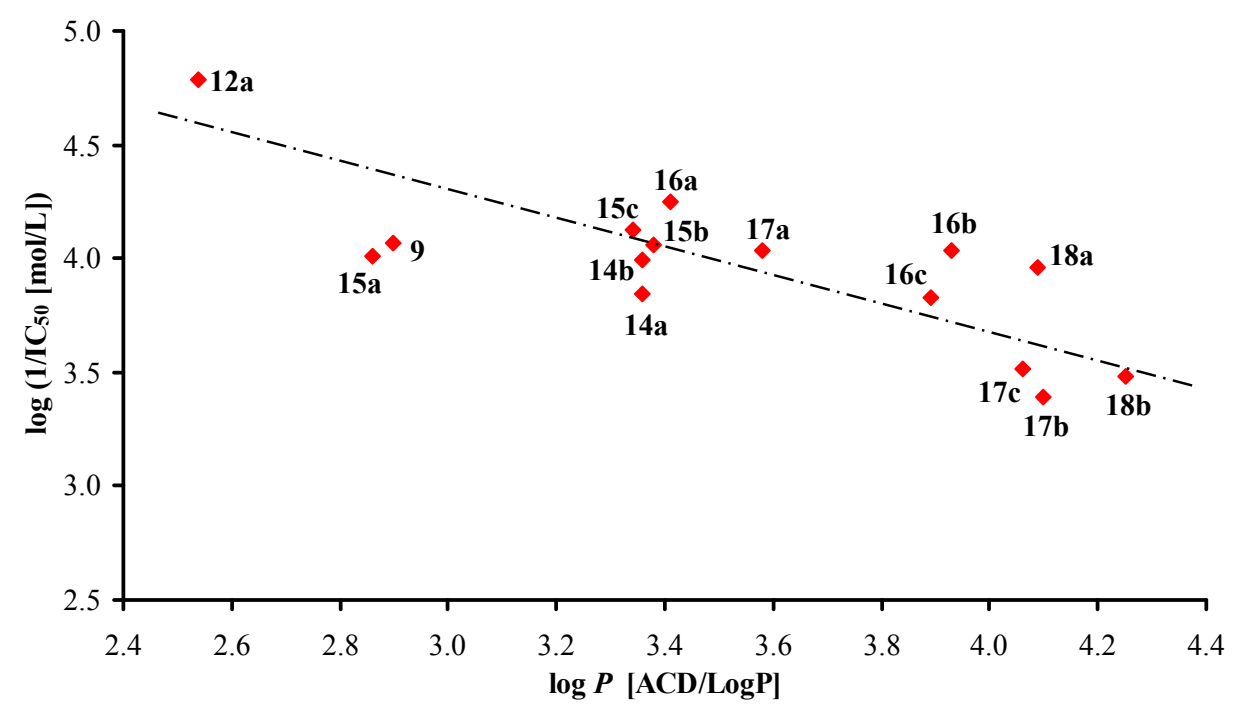

(a)

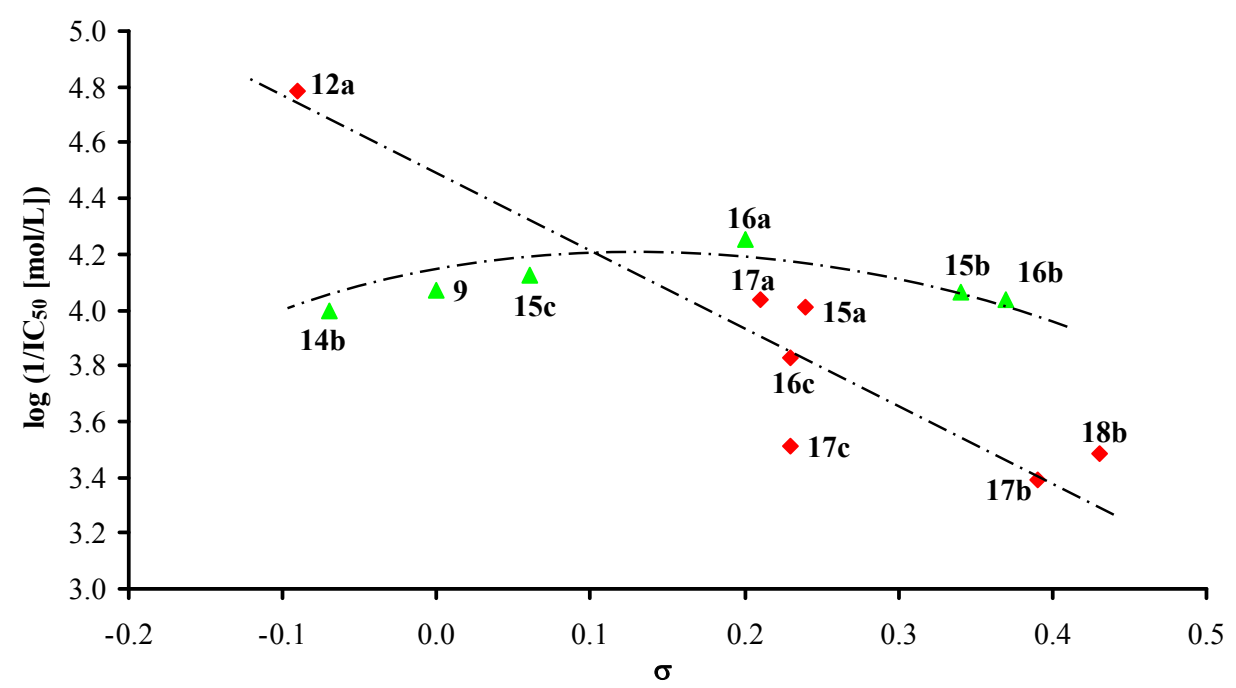

(b) 
Figure 3. Relationships between PET-inhibition $\log \left(1 / \mathrm{IC}_{50}\right)[\mathrm{mol} / \mathrm{L}]$ in spinach chloroplasts and lipophilicity $(\mathbf{a}, \mathbf{b})$ or $N$-substituent electronic $\sigma$ parameters $(\mathbf{c})$ of studied naphthalene-2-carboxamides 20-38c.

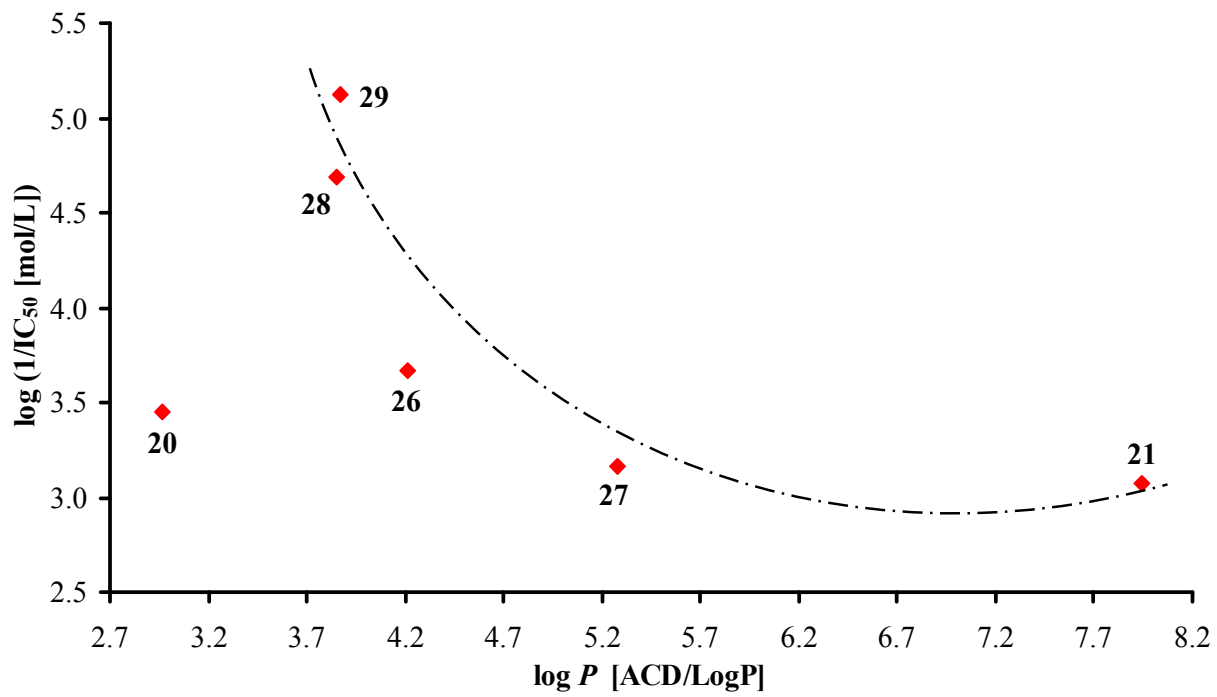

(a)

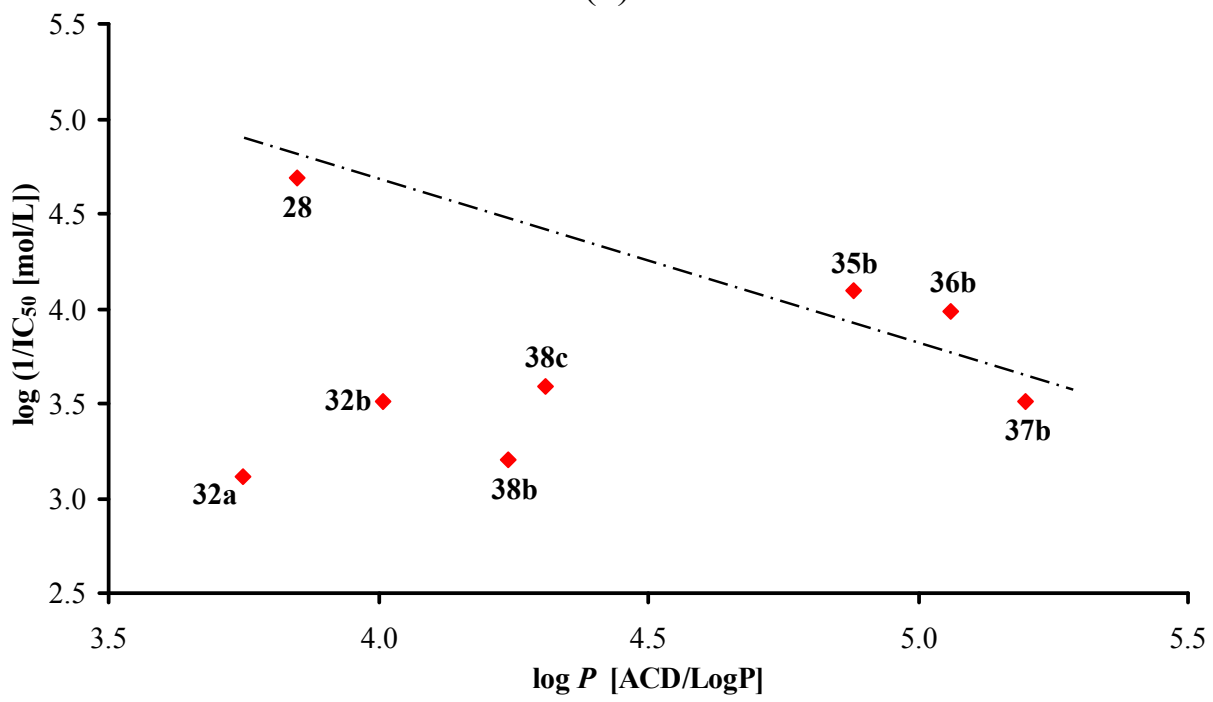

(b)

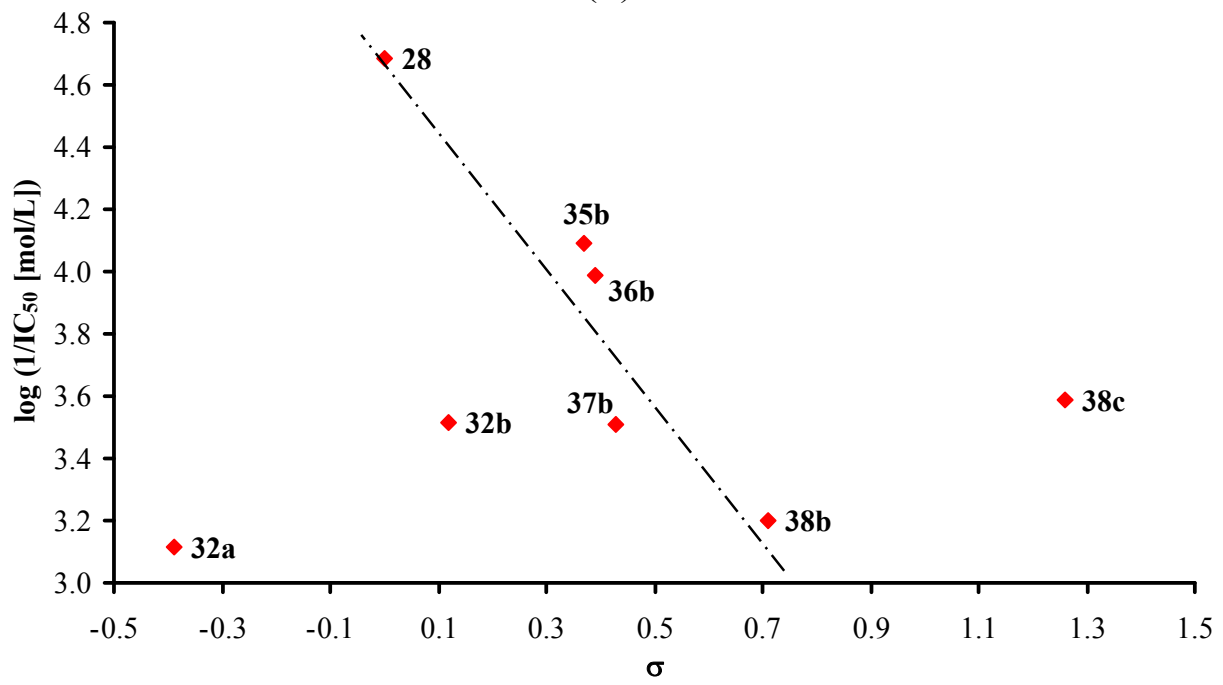

(c) 
In general, the dependence of $\log \left(1 / \mathrm{IC}_{50}\right)$ on $\sigma$ reflects two trends. The first, i.e., compounds with extreme electron $\sigma$ effects, show a similar trend as in case of lipophilicity and thus, the activity decreases with increasing $\sigma$ value. The second trend is a parabolic course with an optimum activity for compound 16a $(2-\mathrm{Cl}-\mathrm{Ph} ; \sigma=0.20)$. In this case the $\sigma$ values of the corresponding compounds ranged from -0.07 to 0.37 , but the differences between activities of the compounds expressed as $\mathrm{IC}_{50}$ values were relatively low and they ranged from 100.6 (14b) to $56.3 \mu \mathrm{mol} / \mathrm{L}$ (16a). However, the importance of the lipophilicity of the anilide substituent was unambiguously much more significant for the inhibitory activity $\left(\mathrm{IC}_{50}[\mathrm{~mol} / \mathrm{L}]\right)$ of the studied compounds than the electronic properties of the substituent.

The most active compound from the series Group 1 was $12 \mathbf{a}\left(\mathrm{R}=2-\mathrm{OH}, \mathrm{IC}_{50}=16.3 \mu \mathrm{mol} / \mathrm{L}\right)$. The result indicates that PET inhibition could be associated with additional interaction of the phenol moiety with photosynthetic proteins. This compound can be understood as a bioisoster of 2-[(2-hydroxyphenylimino)methyl]quinolin-8-ol that expresses high herbicide effect [18].

Generally, the activity of the evaluated naphthalene derivatives (Group 2) related to PET inhibition in spinach (Spinacia oleracea L.) chloroplasts seems to be higher than that of the corresponding quinoline isosters (Table 2). The PET inhibition of 34 of the 68 compounds could not be determined due to their precipitation during the experiments. With respect to these small but closed specifically substituted groups of compounds some structure-activity relationships (SAR) can be proposed.

Figure 3a illustrates the exponential decay of PET-inhibiting activity on the lipophilicity increase of all naphthalene derivatives (compounds 20, 21, 26-29) except substituted phenyl moieties. Dependence of the activity on the electronic properties could not be performed due to small amount of data. As mentioned above, PET-inhibiting activity is not influenced by bulkiness of the $N$-substituents. Benzyl derivative 29 showed higher activity $\left(\mathrm{IC}_{50}=7.5 \mu \mathrm{mol} / \mathrm{L}\right)$ than unsubstituted phenyl derivative 28 $\left(\mathrm{IC}_{50}=20.7 \mu \mathrm{mol} / \mathrm{L}\right)$, similarly to quinolinecarboxamides $\left[\mathrm{IC}_{50}=59.4 \mu \mathrm{mol} / \mathrm{L}(\mathbf{1 0})\right.$ and $85.1 \mu \mathrm{mol} / \mathrm{L}(\mathbf{9})$, respectively].

Dependence of PET inhibition on the lipophilicity of all compounds with the aromatic $N$-substituents is shown on Figure 3b. It is evident that unsubstituted phenyl $\mathbf{2 8}$ derivative expressed the highest PET inhibition; and this decreases as lipophilicity increases. This is observed for lipophilic meta-substituted derivatives $\mathbf{3 5 b}(3-\mathrm{Cl}), \mathbf{3 6} \mathbf{b}(3-\mathrm{Br})$ and $\mathbf{3 7} \mathbf{b}\left(3-\mathrm{CF}_{3}\right)$, contrary to the quinaldinanilides, and it is not valid for methoxy and nitro moieties $(\mathbf{3 2 a} / \mathbf{b}, \mathbf{3 8 b} / \mathbf{c})$. This lower inhibitory activity of compounds 32a $\left(2-\mathrm{OCH}_{3}-\mathrm{Ph}\right), 32 \mathbf{b}\left(2-\mathrm{OCH}_{3}-\mathrm{Ph}\right)$ and 38c $\left(4-\mathrm{NO}_{2}-\mathrm{Ph}\right)$ could be a result of the limited solubility of the compounds, which is typical for $-\mathrm{OCH}_{3}$ and $-\mathrm{NO}_{2}$ substituents. Figure $3 \mathrm{c}$ shows PET inhibition of naphtanilides on Hammett's $\sigma$ parameters of the individual substituents. Based on the results from Figure 3c it can be concluded that PET-inhibiting activity is strongly decreased by the electron-withdrawing effect of substituents in the anilide part of the molecule: $\mathbf{2 9}(\mathrm{Ph})>>\mathbf{3 5 b}(3-\mathrm{Cl})>$ 36b $(3-\mathrm{Br})>37 \mathbf{b}\left(3-\mathrm{CF}_{3}\right)>\mathbf{3 8 b}\left(3-\mathrm{NO}_{3}\right)$, especially in meta-position, contrary to the SAR of quinaldinanilides discussed above.

\subsubsection{In Vitro Antimycobacterial Evaluation}

Although all the compounds were evaluated for their in vitro antimycobacterial activity against M. tuberculosis (clinical isolate with partial INH and PZA resistance) and other atypical mycobacterial strains, most compounds did not show any activity due to their low solubility and precipitation during 
the experiments. Only the 10 compounds presented in Table 3 showed actimycobacterial activities. $N$-Cycloheptylquinoline-2-carboxamide (7) and $N$-(2-phenylethyl)quinoline-2-carboxamide (11) showed high activity against M. tuberculosis, whereas 2-(pyrrolidin-1-ylcarbonyl)quinoline (3) and 1-(2-naphthoyl)pyrrolidine (22) expressed high activity against $M$. avium paratuberculosis and M. kansasii. Compounds 6, 1 and 32a also showed noteworthy activity. Nevertheless, no thorough structure-activity relationships could be established.

According to the results, it can be generally concluded that quinaldinamides (Group 1) possessed higher activity than corresponding naphtamides (Group 2), and anilides seem to be less effective than amides, e.g., highly effective compound 11 and non-active phenyl derivative 9 (not-discussed). Figure 4 shows dependence of the average of antituberculosis/antimycobacterial activities expressed as $\log 1 / \mathrm{MIC}[\mathrm{mol} / \mathrm{L}]$ on lipophilicity expressed as $\log P$. Based on these results, it can be concluded that activity increases as lipophilicity increases. Also, it seems that the increase in antituberculosis activity is connected with the increase in the bulkiness of individual $N$-substituents within the series of cycloalkane, (i.e., cycloheptyl $7>$ cyclohexyl $6>$ cyclopentyl 5). As cyclooctyl derivative 8 demonstrated no activity, it can be concluded that cycloheptyl compound 7 showed the maximum antituberculosis efficacy within this type of compound and under these testing conditions. This decrease might have also actually been caused by decreased solubility of compound $\mathbf{8}$ in the test media (precipitation occurred). It can be speculated that substituents which are bulkier than cyclooctyl could further potentiate the antimycobacterial activity. But the testing conditions for these more lipophilic compounds would have to be changed to prevent the precipitation during the dilution of samples.

Figure 4. Dependence of in vitro average antimycobacterial activity against Mycobacterium sp. $(\log 1 / \mathrm{MIC}[\mathrm{mol} / \mathrm{L}])$ on lipophilicity expressed as $\log P$ of the studied quinaldinamides. (red rhomb $=$ branched alkyl quinaldinamides, green triangle $=$ unbranched long-chain alkyl quinaldinamide, blue square $=$ aromatic quinaldinamides, black point $=$ naphtamides).

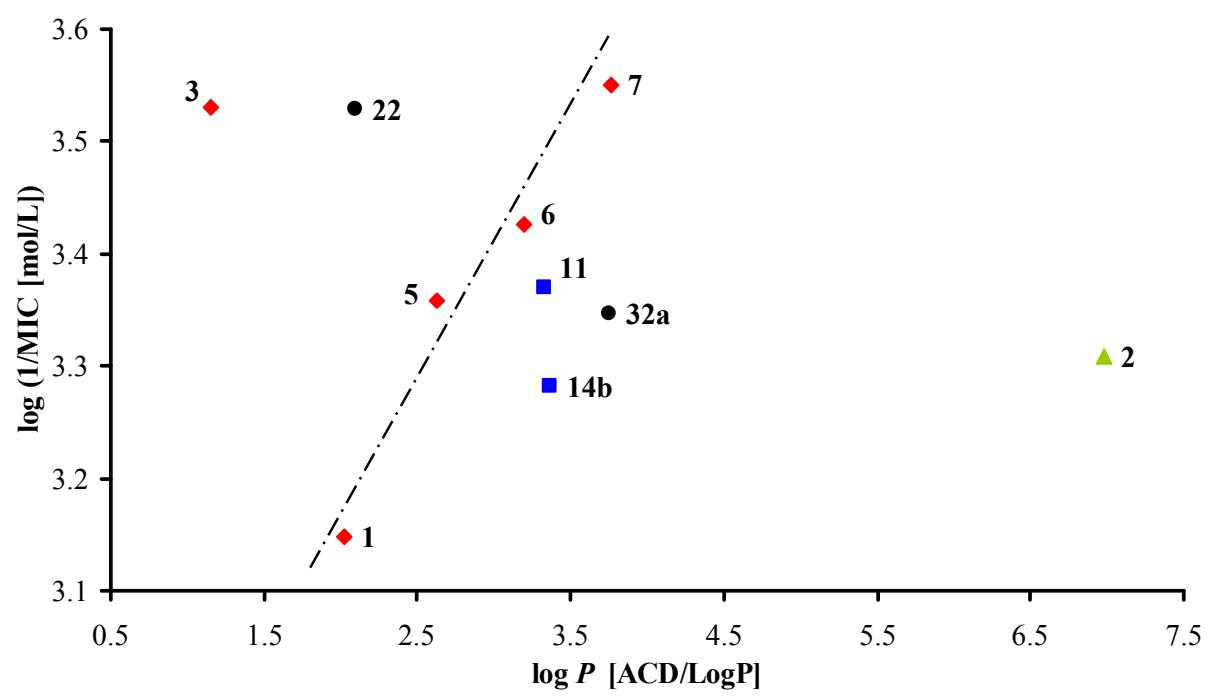

Branching within the $N$-substituents seems also very important, especially from the point of view of antitubercular versus antimycobacterial activity. Unbranched long-chain alkyl dodecyl compound $\mathbf{2}$ or ethylphenyl one $\mathbf{1 1}$ as well as isopropyl compound $\mathbf{1}$ and other cycloalkyl moietis branched in the $\alpha$ position of individual $N$-substituents, seem to be the most advantageous for antituberculosis activity, 
while $N, N$-disubstitution, e.g., compounds $\mathbf{3}$ and 22, is fundamental for high efficacy against the atypical mycobacterial strains $M$. avium paratuberculosis and M. kansasii.

\subsubsection{In Vitro Cytotoxicity Assay}

The most effective antimycobacterial compounds 11, 7, 3 and 22 were tested for their in vitro cytotoxicity $\mathrm{LD}_{50}(\mu \mathrm{mol} / \mathrm{L})$, and subsequently the Selectivity Index, i.e., the ratio of cell toxicity $\left(\mathrm{LD}_{50}\right)$ to activity (MIC), was obtained; the results are presented in Table 3 . The $\mathrm{LD}_{50}$ exact values of compounds 3, 11 and $\mathbf{2 2}$ could not be determined due to their limited solubility and their precipitation from solution during the tests in concentration higher than $100 \mu \mathrm{mol} / \mathrm{L}$, but the highest dose achieved in the medium $(100 \mu \mathrm{mol} / \mathrm{L})$ did not lead to the $100 \%$ lethal effect on THP-1 cells. All the evaluated compounds demonstrated low toxicity against the human monocytic leukemia THP-1 cell line (e.g., $\mathrm{LD}_{50}$ of oxaliplatin $1.7 \pm 6.4$ and camptothecin $0.16 \pm 0.07$ assessed in this line formerly showed much lower values). It can be drawn from Table 3 that compounds 3, 7 and $\mathbf{1 1}$ from Group 1 and compound 22 from Group 2 showed the highest inhibition activity and also moderate cytotoxicity against THP-1 cells; compound 7 expressed the highest toxicity within the series of compounds, but it can not be considered toxic as its $\operatorname{LD}_{50}$ value $(62 \pm 4.5)$ is fairly high. Based on these observations it can be concluded that the discussed amides 11, 3 and 22 can be considered as promising agents for subsequent design of novel antitubercular/antimycobacterial agents.

\section{Experimental}

\subsection{General}

All reagents were purchased from Aldrich. Kieselgel 60, 0.040-0.063 mm (Merck, Darmstadt, Germany) was used for column chromatography. TLC experiments were performed on alumina-backed silica gel 40 F254 plates (Merck, Darmstadt, Germany). The plates were illuminated under UV (254 nm) and evaluated in iodine vapour. The melting points were determined on Kofler hot-plate apparatus HMK (Franz Kustner Nacht KG, Dresden, Germany) and are uncorrected. Infrared (IR) spectra were recorded on a Smart MIRacle ${ }^{\mathrm{TM}}$ ATR ZnSe for Nicolet ${ }^{\mathrm{TM}}$ Impact 410 FT-IR spectrometer (Thermo Scientific, USA). The spectra were obtained by accumulation of 256 scans with $2 \mathrm{~cm}^{-1}$ resolution in the region of 4,000-600 $\mathrm{cm}^{-1}$. All ${ }^{1} \mathrm{H}$ and ${ }^{13} \mathrm{C}$ NMR spectra were recorded on a Bruker Avance III $400 \mathrm{MHz}$ FT-NMR spectrometer $\left(400 \mathrm{MHz}\right.$ for ${ }^{1} \mathrm{H}$ and $100 \mathrm{MHz}$ for ${ }^{13} \mathrm{C}$, Bruker Co., Karlsruhe, Germany). Chemicals shifts are reported in ppm $(\delta)$ using internal $\mathrm{Si}\left(\mathrm{CH}_{3}\right)_{4}$ as the reference with diffuse, easily exchangeable signals being omitted. Mass spectra were measured using a LTQ Orbitrap Hybrid Mass Spectrometer (Thermo Electron Corporation, USA) with direct injection into an APCI source $\left(400^{\circ} \mathrm{C}\right)$ in the positive mode.

\subsection{Synthesis}

\subsubsection{General Procedure for Synthesis of Carboxamide Derivatives 1-19c}

2-Quinaldic acid (1 g, $5.8 \mathrm{mmol})$ was suspended in dry toluene $(15 \mathrm{~mL})$ at room temperature and oxalyl chloride ( $1 \mathrm{~mL}, 1.61 \mathrm{~g}, 12.7 \mathrm{mmol}, 2.2$ eq.) was added dropwise. The reaction mixture was 
stirred for 30 min at the same temperature and then DMF (2 drops) was added. The mixture was stirred for $24 \mathrm{~h}$ and then evaporated to dryness. The residue was washed with petroleum ether and used directly in the next step. Into the solution of 2-quinaldic acid chloride in dry toluene (15 $\mathrm{mL})$, triethylamine $(4.5 \mathrm{~mL}, 2.92 \mathrm{~g}, 32.5 \mathrm{mmol})$ and corresponding substituted aniline $(5.8 \mathrm{mmol})$ were added dropwise. The mixture was stirred at room temperature for $24 \mathrm{~h}$ after which the solvent was removed under reduced pressure. The residue was extracted with $\mathrm{CHCl}_{3}$. Combined organic layers were washed with water and saturated aqueous solution of $\mathrm{NaHCO}_{3}$ and dried over anhydrous $\mathrm{MgSO}_{4}$. The solvent was evaporated to dryness under reduced pressure. The crude product was recrystallized from isopropanol or EtOAc. The studied compounds 1-19c are presented in Table 1.

N-Isopropylquinoline-2-carboxamide (1). Yield 40\%; Mp. $75-76{ }^{\circ} \mathrm{C}$; IR ( $\mathrm{Zn} / \mathrm{Se}$ ATR, $\left.\mathrm{cm}^{-1}\right): 3,339 m$, $3,052 w, 2,925 w, 2,852 w, 1,676 s, 1,589 m, 1,556 m, 1,525 s, 1,501 m, 1,434 w, 1,418 w, 1,205 w, 1,119 m$, $903 w, 841 w, 770 m, 747 m, 686 w, 666 w ;{ }^{1} \mathrm{H}-\mathrm{NMR}$ (DMSO- $\left.d_{6}\right), \delta: 8.60(\mathrm{~d}, J=8.0 \mathrm{~Hz}, 1 \mathrm{H}), 8.54(\mathrm{~d}$, $J=8.5 \mathrm{~Hz}, 1 \mathrm{H}), 8.16(\mathrm{~d}, J=8.5 \mathrm{~Hz}, 2 \mathrm{H}), 8.05(\mathrm{~d}, J=8.3 \mathrm{~Hz}, 1 \mathrm{H}), 7.80-7.89(\mathrm{~m}, 1 \mathrm{H}), 7.64-7.74(\mathrm{~m}$, $1 \mathrm{H}), 4.03-4.28(\mathrm{~m}, 1 \mathrm{H}), 1.23(\mathrm{~d}, J=6.8 \mathrm{~Hz}, 6 \mathrm{H}) ;{ }^{13} \mathrm{C}-\mathrm{NMR}$ (DMSO- $\left.d_{6}\right), \delta: 163.02,150.32,145.96$, 137.83, 130.45, 129.18, 128.76, 128.07, 127.98, 118.65, 40.94, 22.27; HR-MS: for $\mathrm{C}_{13} \mathrm{H}_{15} \mathrm{~N}_{2} \mathrm{O}[\mathrm{M}+\mathrm{H}]^{+}$ calculated $215.1179 \mathrm{~m} / \mathrm{z}$, found $215.1139 \mathrm{~m} / \mathrm{z}$.

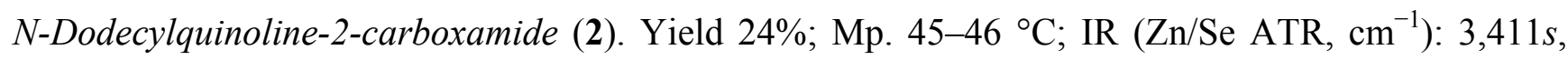
$3,052 w, 2,983 w, 2,901 w, 1,671 s, 1,589 w, 1,561 m, 1,521 m, 1,492 m, 1,450 w, 1,423 s, 1,201 w, 1,131 w$, $1,107 w, 1,074 w, 1,054 w, 907 w, 841 m, 768 w, 735 m, 696 m ;{ }^{1} \mathrm{H}-\mathrm{NMR}$ (DMSO- $d_{6}$ ), $\delta: 8.88$ (t, $J=6.0 \mathrm{~Hz}, 1 \mathrm{H}), 8.53(\mathrm{~d}, J=8.3 \mathrm{~Hz}, 1 \mathrm{H}), 8.15(\mathrm{~d}, J=8.5 \mathrm{~Hz}, 1 \mathrm{H}), 8.11(\mathrm{~d}, J=8.5 \mathrm{~Hz}, 1 \mathrm{H}), 8.05(\mathrm{~d}$, $J=8.0 \mathrm{~Hz}, 1 \mathrm{H}), 7.81-7.86(\mathrm{~m}, 1 \mathrm{H}), 7.65-7.71(\mathrm{~m}, 1 \mathrm{H}), 3.31-3.38(\mathrm{~m}, 2 \mathrm{H}), 1.54$ (quin, $J=6.9 \mathrm{~Hz}$, $2 \mathrm{H}), 1.10-1.31(\mathrm{~m}, 18 \mathrm{H}), 0.78(\mathrm{t}, J=6.9 \mathrm{~Hz}, 3 \mathrm{H}) ;{ }^{13} \mathrm{C}-\mathrm{NMR}$ (DMSO- $\left.d_{6}\right), \delta: 163.78,150.29,145.99$, 137.72 , 130.37, 129.13, 128.76, 128.04, 127.89, 118.60, 38.96, 31.30, 29.24, 29.06, 29.03, 29.02, 29.00, 28.81, 28.73, 26.49, 22.09, 13.89; HR-MS: for $\mathrm{C}_{22} \mathrm{H}_{33} \mathrm{~N}_{2} \mathrm{O}[\mathrm{M}+\mathrm{H}]^{+}$calculated $341.2587 \mathrm{~m} / z$, found $341.2599 \mathrm{~m} / z$.

2-(Pyrrolidin-1-ylcarbonyl)quinoline (3) [39]. Yield 41\%; Mp. 71-72 ${ }^{\circ} \mathrm{C}$; IR (Zn/Se ATR, $\left.\mathrm{cm}^{-1}\right)$ : 3,291m, 3,064w, 2,919s, 2,844w, 1,644s, 1,561w, 1,532s, 1,498m, 1,467m, 1,438w, 1,422m, 1,344w, $1,324 w, 1,201 w, 1,168 m, 1,070 m, 1,009 w, 903 m, 842 m, 774 s, 735 m, 678 m ;{ }^{1} \mathrm{H}-\mathrm{NMR}$ (DMSO- $d_{6}$ ), $\delta: 8.46(\mathrm{~d}, J=8.5 \mathrm{~Hz}, 1 \mathrm{H}), 8.03(\mathrm{t}, J=9.2 \mathrm{~Hz}, 2 \mathrm{H}), 7.77-7.83(\mathrm{~m}, 2 \mathrm{H}), 7.66$ (ddd, $J=8.0 \mathrm{~Hz}$, $J=6.9 \mathrm{~Hz}, J=1.1 \mathrm{~Hz}, 1 \mathrm{H}), 3.67(\mathrm{t}, J=6.4 \mathrm{~Hz}, 2 \mathrm{H}), 3.55(\mathrm{t}, J=6.5 \mathrm{~Hz}, 2 \mathrm{H}), 1.78-1.89(\mathrm{~m}, 4 \mathrm{H})$; ${ }^{13} \mathrm{C}-\mathrm{NMR}$ (DMSO- $d_{6}$ ), $\delta: 165.56,154.22,145.87,137.07,130.14,129.15,127.90,127.75,127.64$, 120.41, 48.46, 46.39, 26.05, 23.59; HR-MS: for $\mathrm{C}_{14} \mathrm{H}_{15} \mathrm{~N}_{2} \mathrm{O}[\mathrm{M}+\mathrm{H}]^{+}$calculated $227.1179 \mathrm{~m} / z$, found $227.1203 \mathrm{~m} / \mathrm{z}$.

2-(Piperidin-1-ylcarbonyl)quinoline (4) [40]. Yield 59\%; Mp. 83-84 ${ }^{\circ} \mathrm{C}$; IR (Zn/Se ATR, $\left.\mathrm{cm}^{-1}\right)$ : $3,327 s, 3,023 w, 2,934 w, 1,655 s, 1,561 w, 1,522 m, 1,495 m, 1,454 m, 1,426 s, 1,360 m, 1,331 w, 1,230 w$, $1,209 w, 1,156 m, 1,074 w, 837 m, 772 w, 735 m, 701 m ;{ }^{1} \mathrm{H}-\mathrm{NMR}$ (DMSO- $\left.d_{6}\right), \delta: 8.48(\mathrm{~d}, J=8.3 \mathrm{~Hz}, 1 \mathrm{H})$, 7.96-8.07 (m, 2H), 7.81 (ddd, $J=8.5 \mathrm{~Hz}, J=6.8 \mathrm{~Hz}, J=1.5 \mathrm{~Hz}, 1 \mathrm{H}), 7.57-7.71(\mathrm{~m}, 2 \mathrm{H}), 3.61-3.69$ $(\mathrm{m}, 2 \mathrm{H}), 3.22-3.35(\mathrm{~m}, 2 \mathrm{H}), 1.38-1.73(\mathrm{~m}, 6 \mathrm{H}) ;{ }^{13} \mathrm{C}-\mathrm{NMR}$ (DMSO- $\left.d_{6}\right), \delta: 166.63,154.67,146.14$, 
137.34, 130.26, 128.99, 128.00, 127.46, 127.38, 120.04, 47.45, 42.22, 25.97, 25.31, 23.97; HR-MS: for $\mathrm{C}_{15} \mathrm{H}_{17} \mathrm{~N}_{2} \mathrm{O}[\mathrm{M}+\mathrm{H}]^{+}$calculated $241.1335 \mathrm{~m} / \mathrm{z}$, found $241.1384 \mathrm{~m} / \mathrm{z}$.

N-Cyclopentylquinoline-2-carboxamide (5). Yield 36\%; Mp. $63-64{ }^{\circ} \mathrm{C}$; IR (Zn/Se ATR, cm $\left.{ }^{-1}\right)$ : 3,387m, 3,305m, 2,933m, 2,848m, 1,655s, 1,614w, 1,561w, 1,524s, 1,497s, 1,446m, 1,373w, 1,205w, $1,160 m, 1,136 m, 1,074 w, 849 m, 796 w, 779 s, 735 w ;{ }^{1} \mathrm{H}-\mathrm{NMR}$ (DMSO- $\left.d_{6}\right), \delta: 8.68(\mathrm{~d}, J=8.0 \mathrm{~Hz}, 1 \mathrm{H})$, $8.54(\mathrm{~d}, J=8.5 \mathrm{~Hz}, 1 \mathrm{H}), 8.15(\mathrm{dd}, J=8.4 \mathrm{~Hz}, J=2.6 \mathrm{~Hz}, 2 \mathrm{H}), 8.06(\mathrm{~d}, J=8.0 \mathrm{~Hz}, 1 \mathrm{H}), 7.85(\mathrm{dd}$, $J=8.3 \mathrm{~Hz}, J=7.0 \mathrm{~Hz}, 1 \mathrm{H}), 7.65-7.74(\mathrm{~m}, 1 \mathrm{H}), 4.30$ (sxt, $J=7.3 \mathrm{~Hz}, 1 \mathrm{H}), 1.85-2.01(\mathrm{~m}, 2 \mathrm{H})$, $1.48-1.74(\mathrm{~m}, 6 \mathrm{H}) ;{ }^{13} \mathrm{C}-\mathrm{NMR}$ (DMSO- $\left.d_{6}\right), \delta: 163.59,150.30,145.97,137.85,130.46,129.22,128.77$, 128.09, 128.00, 118.66, 50.72, 32.17, 23.60; HR-MS: for $\mathrm{C}_{15} \mathrm{H}_{17} \mathrm{~N}_{2} \mathrm{O}[\mathrm{M}+\mathrm{H}]^{+}$calculated $241.1335 \mathrm{~m} / z$, found $241.1375 \mathrm{~m} / \mathrm{z}$.

$\mathrm{N}$-Cyclohexylquinoline-2-carboxamide (6) [40]. Yield 40\%; Mp. 98-99 ${ }^{\circ} \mathrm{C}$; IR (Zn/Se ATR, $\mathrm{cm}^{-1}$ ): $3,329 m, 3,313 m, 2,969 s, 2,933 m, 2,875 m, 1,648 s, 1,556 w, 1,522 s, 1,498 s, 1,470 m, 1,423 s, 1,382 w$, $1,361 m, 1,341 w, 1,324 w, 1,201 w, 1,178 m, 1,130 m, 1,105 w, 903 w, 844 m, 776 w, 735 w, 674 w ;{ }^{1} \mathrm{H}-\mathrm{NMR}$ (DMSO-d $\left.d_{6}\right), \delta: 8.57(\mathrm{~d}, J=8.5 \mathrm{~Hz}, 1 \mathrm{H}), 8.54(\mathrm{~d}, J=8.5 \mathrm{~Hz}, 1 \mathrm{H}), 8.16(\mathrm{~d}, J=8.3 \mathrm{~Hz}, 2 \mathrm{H}), 8.06(\mathrm{~d}$, $J=8.0 \mathrm{~Hz}, 1 \mathrm{H}), 7.81-7.88(\mathrm{~m}, 1 \mathrm{H}), 7.66-7.72(\mathrm{~m}, 1 \mathrm{H}), 3.78-3.89(\mathrm{~m}, 1 \mathrm{H}), 1.06-1.88(\mathrm{~m}, 10 \mathrm{H})$;

${ }^{13} \mathrm{C}-\mathrm{NMR}$ (DMSO- $d_{6}$ ), $\delta: 162.92,150.27,145.94,137.84,130.44,129.20,128.77,128.06,127.98$, 118.64, 48.12, 32.26, 25.12, 24.83; HR-MS: for $\mathrm{C}_{16} \mathrm{H}_{19} \mathrm{~N}_{2} \mathrm{O}[\mathrm{M}+\mathrm{H}]^{+}$calculated $255.1492 \mathrm{~m} / z$, found $255.1525 \mathrm{~m} / \mathrm{z}$.

N-Cycloheptylquinoline-2-carboxamide (7). Yield 39\%; Mp. $72-73{ }^{\circ} \mathrm{C}$; IR (Zn/Se ATR, cm ${ }^{-1}$ ): $3,056 w, 2,958 m, 2,864 m, 1,615 s, 1,552 m, 1,469 m, 1,436 m, 1,417 s, 1,373 w, 1,332 w, 1,205 w, 1,181 w$, $1,156 w, 842 m, 764 m, 731 w, 657 w ;{ }^{1} \mathrm{H}-\mathrm{NMR}$ (DMSO-d $\left.d_{6}\right), \delta: 8.61(\mathrm{~d}, J=8.5 \mathrm{~Hz}, 1 \mathrm{H}), 8.54(\mathrm{~d}$, $J=8.3 \mathrm{~Hz}, 1 \mathrm{H}), 8.15(\mathrm{~d}, J=8.5 \mathrm{~Hz}, 2 \mathrm{H}), 8.06(\mathrm{~d}, J=7.5 \mathrm{~Hz}, 1 \mathrm{H}), 7.85(\mathrm{ddd}, J=8.4 \mathrm{~Hz}, J=6.9 \mathrm{~Hz}$, $J=1.3 \mathrm{~Hz}, 1 \mathrm{H}), 7.69$ (ddd, $J=8.0 \mathrm{~Hz}, J=6.9 \mathrm{~Hz}, J=1.1 \mathrm{~Hz}, 1 \mathrm{H}), 4.01$ (qt, $J=9.0 \mathrm{~Hz}, J=4.5 \mathrm{~Hz}$, 1H), 1.81-1.93 (m, 2H), 1.37-1.72 (m, 10H); ${ }^{13} \mathrm{C}-\mathrm{NMR}$ (DMSO-d $)$, $\delta: ~ 162.64,150.30,145.94$, $137.85,130.45,129.21,128.78,128.06,127.98,118.63,50.32,34.30,27.57,23.88$; HR-MS: for $\mathrm{C}_{17} \mathrm{H}_{21} \mathrm{~N}_{2} \mathrm{O}[\mathrm{M}+\mathrm{H}]^{+}$calculated $269.1648 \mathrm{~m} / \mathrm{z}$, found $269.1619 \mathrm{~m} / \mathrm{z}$.

N-Cyclooctylquinoline-2-carboxamide (8) [41]. Yield 44\%; Mp. 72-73 ${ }^{\circ} \mathrm{C}$; IR (Zn/Se ATR, cm ${ }^{-1}$ ): $3,301 w, 2,954 m, 2,916 s, 2,848 s, 1,667 s, 1,649 s, 1,593 w, 1,532 s, 1,498 s, 1,462 m, 1,418 m, 1,373 w$, $1,340 w, 1,315 w, 1,295 m, 1,209 w, 1,168 m, 1136 w, 1,111 w, 903 w, 850 s, 796 w, 772 s, 735 m, 715 w$, $666 w ;{ }^{1} \mathrm{H}-\mathrm{NMR}\left(\mathrm{DMSO}-d_{6}\right), \delta: 8.59(\mathrm{~d}, J=7.8 \mathrm{~Hz}, 1 \mathrm{H}), 8.55(\mathrm{~d}, J=8.5 \mathrm{~Hz}, 1 \mathrm{H}), 8.15(\mathrm{~d}, J=8.3 \mathrm{~Hz}$, $2 \mathrm{H}), 8.06(\mathrm{~d}, J=8.0 \mathrm{~Hz}, 1 \mathrm{H}), 7.81-7.88(\mathrm{~m}, 1 \mathrm{H}), 7.66-7.73(\mathrm{~m}, 1 \mathrm{H}), 4.00-4.12(\mathrm{~m}, 1 \mathrm{H}), 1.45-1.81$ $(\mathrm{m}, 14 \mathrm{H}) ;{ }^{13} \mathrm{C}-\mathrm{NMR}$ (DMSO- $\left.d_{6}\right), \delta: 162.60,150.32,145.94,137.85,130.45,129.21,128.78,128.06$, 127.98, 118.61, 49.11, 31.80, 26.71, 25.00, 23.50; HR-MS: for $\mathrm{C}_{18} \mathrm{H}_{23} \mathrm{~N}_{2} \mathrm{O}[\mathrm{M}+\mathrm{H}]^{+}$calculated $283.1805 \mathrm{~m} / \mathrm{z}$, found $283.1777 \mathrm{~m} / \mathrm{z}$.

N-Phenylquinoline-2-carboxamide (9). Yield 59\%; Mp. 139-140 ${ }^{\circ} \mathrm{C}$ (Mp. 139.5-140 ${ }^{\circ} \mathrm{C}$ [42]); IR $\left(\mathrm{Zn} / \mathrm{Se}\right.$ ATR, $\left.\mathrm{cm}^{-1}\right): 3,293 m, 2,927 s, 2,855 m, 1,643 s, 1,562 m, 1,530 s, 1,500 s, 1,426 m, 1,315 w, 1,205 w$, $1,185 w, 1,160 w, 1,070 w, 1,042 w, 910 m, 870 w, 841 m, 776 m, 731 w ;{ }^{1} \mathrm{H}-\mathrm{NMR}$ (DMSO- $d_{6}$ ), $\delta: 10.75$ (bs, $1 \mathrm{H}), 8.62(\mathrm{~d}, J=8.5 \mathrm{~Hz}, 1 \mathrm{H}), 8.22-8.30(\mathrm{~m}, 2 \mathrm{H}), 8.11(\mathrm{~d}, J=7.5 \mathrm{~Hz}, 1 \mathrm{H}), 7.94-7.99$ (m, 2H), 7.91 
(ddd, $J=8.5 \mathrm{~Hz}, J=7.0 \mathrm{~Hz}, J=1.4 \mathrm{~Hz}, 1 \mathrm{H}), 7.75(\mathrm{ddd}, J=8.1 \mathrm{~Hz}, J=7.0 \mathrm{~Hz}, J=1.3 \mathrm{~Hz}, 1 \mathrm{H}$ ), 7.38-7.45 (m, 2H), 7.12-7.19 (m, 1H); ${ }^{13} \mathrm{C}-\mathrm{NMR}$ (DMSO- $d_{6}$ ), $\delta: 162.71,150.08,145.88,138.31$, $138.22,130.68,129.35,128.94,128.78,128.37,128.15,124.05,120.29,118.77$; HR-MS: for $\mathrm{C}_{16} \mathrm{H}_{13} \mathrm{~N}_{2} \mathrm{O}[\mathrm{M}+\mathrm{H}]^{+}$calculated $249.1022 \mathrm{~m} / \mathrm{z}$, found $249.1015 \mathrm{~m} / z$.

N-Benzylquinoline-2-carboxamide (10). Yield 60\%; Mp. 123-124 ${ }^{\circ} \mathrm{C}$ (Mp. 124-125 ${ }^{\circ} \mathrm{C}$ [43]); IR (Zn/Se ATR, $\left.\mathrm{cm}^{-1}\right): 3,273 m, 2,952 m, 2,856 m, 1,657 s, 1,643 s, 1,589 w, 1,562 m, 1,527 s, 1,498 s, 1,446 w$, $1,424 m, 1,315 w, 1,299 w, 1,205 m, 1,181 w, 1,152 w, 1,143 w, 956 w, 899 m, 846 s, 792 m, 770 m, 776 m$, $739 m, 686 m$; ${ }^{1} \mathrm{H}-\mathrm{NMR}$ (DMSO- $\left.d_{6}\right), \delta: 9.50(\mathrm{t}, J=6.4 \mathrm{~Hz}, 1 \mathrm{H}), 8.56(\mathrm{~d}, J=8.3 \mathrm{~Hz}, 1 \mathrm{H}), 8.19(\mathrm{~d}$, $J=8.5 \mathrm{~Hz}, 1 \mathrm{H}), 8.14(\mathrm{~d}, J=8.5 \mathrm{~Hz}, 1 \mathrm{H}), 8.07(\mathrm{dd}, J=8.3 \mathrm{~Hz}, J=0.8 \mathrm{~Hz}, 1 \mathrm{H}), 7.86(\mathrm{ddd}, J=8.4 \mathrm{~Hz}$, $J=6.9 \mathrm{~Hz}, J=1.5 \mathrm{~Hz}, 1 \mathrm{H}), 7.70(\mathrm{ddd}, J=8.2 \mathrm{~Hz}, J=6.9 \mathrm{~Hz}, J=1.0 \mathrm{~Hz}, 1 \mathrm{H}), 7.36-7.41(\mathrm{~m}, 2 \mathrm{H})$, $7.29-7.35(\mathrm{~m}, 2 \mathrm{H}), 7.20-7.26(\mathrm{~m}, 1 \mathrm{H}), 4.59(\mathrm{~d}, J=6.5 \mathrm{~Hz}, 2 \mathrm{H}) ;{ }^{13} \mathrm{C}-\mathrm{NMR}$ (DMSO-d $), \delta: 164.13$, $150.15,146.04,139.52,137.88,130.52,129.19$, 128.84, 128.30, 128.11, 128.07, 127.46, 126.82, 118.74, 42.56; HR-MS: for $\mathrm{C}_{17} \mathrm{H}_{15} \mathrm{~N}_{2} \mathrm{O}[\mathrm{M}+\mathrm{H}]^{+}$calculated $263.1179 \mathrm{~m} / \mathrm{z}$, found $263.1202 \mathrm{~m} / \mathrm{z}$.

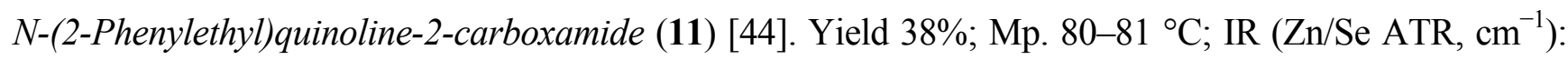
$3,236 w, 2,935 s, 2,852 m, 1,633 s, 1,552 w, 1,467 m, 1,450 m, 1,422 m, 1,344 w, 1,279 m, 1,103 w, 1,025 w$, $1,005 w, 952 w, 890 w, 841 m, 760 m, 731 w, 682 w ;{ }^{1} \mathrm{H}-\mathrm{NMR}$ (DMSO-d $), \delta: 9.00(\mathrm{t}, J=5.90 \mathrm{~Hz}, 1 \mathrm{H})$, $8.54(\mathrm{~d}, J=8.5 \mathrm{~Hz}, 1 \mathrm{H}), 8.17(\mathrm{~d}, J=8.5 \mathrm{~Hz}, 1 \mathrm{H}), 8.11(\mathrm{~d}, J=8.5 \mathrm{~Hz}, 1 \mathrm{H}), 8.06(\mathrm{~d}, J=8.0 \mathrm{~Hz}, 1 \mathrm{H})$, $7.85(\mathrm{td}, J=7.7 \mathrm{~Hz}, J=1.3 \mathrm{~Hz}, 1 \mathrm{H}), 7.66-7.73(\mathrm{~m}, 1 \mathrm{H}), 7.25-7.33(\mathrm{~m}, 4 \mathrm{H}), 7.16-7.22(\mathrm{~m}, 1 \mathrm{H})$, $3.58-3.65(\mathrm{~m}, 2 \mathrm{H}), 2.92(\mathrm{t}, J=7.5 \mathrm{~Hz}, 2 \mathrm{H}) ;{ }^{13} \mathrm{C}-\mathrm{NMR}$ (DMSO- $\left.d_{6}\right), \delta: 163.88,150.14,145.96,139.36$, $137.83,130.48,129.13,128.78,128.64,128.38,128.08,128.00,126.14,118.59,40.58,35.23$; HR-MS: for $\mathrm{C}_{18} \mathrm{H}_{17} \mathrm{~N}_{2} \mathrm{O}[\mathrm{M}+\mathrm{H}]^{+}$calculated $277.1335 \mathrm{~m} / \mathrm{z}$, found $277.1384 \mathrm{~m} / \mathrm{z}$.

$\mathrm{N}$-(2-Hydroxyphenyl)quinoline-2-carboxamide (12a) [45]. Yield 27\%; Mp. 219-220 ${ }^{\circ} \mathrm{C}$; ${ }^{1} \mathrm{H}-\mathrm{NMR}$ (DMSO- $d_{6}$ ), $\delta$ : 10.67 (bs, $\left.1 \mathrm{H}\right), 10.44$ (bs, $\left.1 \mathrm{H}\right), 8.63$ (d, $\left.J=8.5 \mathrm{~Hz}, 1 \mathrm{H}\right), 8.44(\mathrm{~d}, J=7.8 \mathrm{~Hz}, 1 \mathrm{H}), 8.29$ $(\mathrm{d}, J=8.3 \mathrm{~Hz}, 1 \mathrm{H}), 8.15(\mathrm{~d}, J=8.3 \mathrm{~Hz}, 1 \mathrm{H}), 8.10(\mathrm{~d}, J=8.0 \mathrm{~Hz}, 1 \mathrm{H}), 7.88(\mathrm{t}, J=7.3 \mathrm{~Hz}, 1 \mathrm{H}), 7.73(\mathrm{t}$, $J=7.2 \mathrm{~Hz}, 1 \mathrm{H}), 7.00(\mathrm{~m}, 2 \mathrm{H}), 6.89(\mathrm{~m}, 1 \mathrm{H}) ;{ }^{13} \mathrm{C}-\mathrm{NMR}$ (DMSO- $\left.d_{6}\right), \delta: 161.26,149.65,146.77,145.74$, 138.59, 130.91, 129.29, 129.08, 128.46, 128.22, 126.20, 124.29, 119.37, 119.26, 118.42, 114.84; HR-MS: for $\mathrm{C}_{16} \mathrm{H}_{13} \mathrm{~N}_{2} \mathrm{O}_{2}[\mathrm{M}+\mathrm{H}]^{+}$calculated $265.0977 \mathrm{~m} / \mathrm{z}$, found $265.0983 \mathrm{~m} / \mathrm{z}$.

$N$-(3-Hydroxyphenyl)quinoline-2-carboxamide (12b). Yield 52\%; Mp. 154-155 ${ }^{\circ} \mathrm{C}$; IR (Zn/Se ATR, $\left.\mathrm{cm}^{-1}\right): 3,338 w, 2,973 w, 1,760 m, 1,680 m, 1,605 w, 1,530 s, 1,504 s, 1,462 w, 1,425 m, 1,263 m, 1,235 m$, $1,216 m, 1,171 w, 1,145 s, 1,096 m, 1,054 w, 1,002 w, 964 w, 908 m, 879 w, 841 m, 773 s, 754 s, 685 m, 665 m$; ${ }^{1} \mathrm{H}-\mathrm{NMR}$ (DMSO- $d_{6}$ ), $\delta: 10.98$ (bs, $\left.1 \mathrm{H}\right), 8.62(\mathrm{~d}, J=8.7 \mathrm{~Hz}, 1 \mathrm{H}), 8.20-8.36(\mathrm{~m}, 3 \mathrm{H}), 8.06-8.18$ (m, 1H), 7.87-7.97 (m, 2H), 7.71-7.83 (m, 2H), $7.54(\mathrm{t}, J=8.2 \mathrm{~Hz}, 1 \mathrm{H}), 7.18(\mathrm{dd}, J=8.0 \mathrm{~Hz}, J=1.8 \mathrm{~Hz}$, $1 \mathrm{H}$ ); ${ }^{13} \mathrm{C}-\mathrm{NMR}$ (DMSO-d ${ }_{6}$ ), $\delta: 163.05,150.91,149.86,147.01,145.90,139.63,138.29,130.94$, 129.99, 129.19, 128.49, 128.16, 121.35, 118.83, 117.99, 113.65; HR-MS: for $\mathrm{C}_{16} \mathrm{H}_{13} \mathrm{~N}_{2} \mathrm{O}_{2}[\mathrm{M}+\mathrm{H}]^{+}$ calculated $265.0977 \mathrm{~m} / \mathrm{z}$, found $265.0985 \mathrm{~m} / \mathrm{z}$.

$\mathrm{N}$-(4-Hydroxyphenyl)quinoline-2-carboxamide (12c) [46]. Yield 48\%; Mp. 230-231 ${ }^{\circ} \mathrm{C}$; ${ }^{1} \mathrm{H}-\mathrm{NMR}$ (DMSO- $d_{6}$ ), $\delta: 10.34$ (bs, 1H), 8.28-8.48 (m, 3H), 8.21 (d, $\left.J=8.5 \mathrm{~Hz}, 1 \mathrm{H}\right), 7.88-8.04(\mathrm{~m}, 3 \mathrm{H})$, 7.77-7.87 (m, 1H), $7.69(\mathrm{~m}, 1 \mathrm{H}), 7.39$ (d, $J=9.0 \mathrm{~Hz}, 2 \mathrm{H}) ;{ }^{13} \mathrm{C}-\mathrm{NMR}$ (DMSO- $d_{6}$ ), $\delta: 162.11,149.40$, 
147.23, 146.23, 137.94, 135.82, 130.84, 129.62, 129.45, 128.95, 127.60, 122.38, 120.66, 118.73; HR-MS: for $\mathrm{C}_{16} \mathrm{H}_{13} \mathrm{~N}_{2} \mathrm{O}_{2}[\mathrm{M}+\mathrm{H}]^{+}$calculated $265.0977 \mathrm{~m} / \mathrm{z}$, found $265.0971 \mathrm{~m} / \mathrm{z}$.

$N$-(2-Methoxyphenyl)quinoline-2-carboxamide (13a) [47]. Yield 37\%; Mp. $111-112{ }^{\circ} \mathrm{C}$; IR ( $\mathrm{Zn} / \mathrm{Se}$ ATR, $\left.\mathrm{cm}^{-1}\right): 3,382 w, 1,676 s, 1,596 m, 1,532 s, 1,485 w, 1,454 m, 1,426 m, 1,334 w, 1,288 w, 1,253 m$, $1,138 m, 1,129 m, 1,093 w, 1,020 s, 951 w, 908 m, 873 w, 840 m, 820 w, 770 s, 732 s ;{ }^{1} \mathrm{H}-\mathrm{NMR}$ (DMSO- $d_{6}$ ), $\delta$ : 10.68 (bs, 1H), 8.59 (d, $J=8.5 \mathrm{~Hz}, 1 \mathrm{H}), 8.49$ (d, $J=7.8 \mathrm{~Hz}, 1 \mathrm{H}), 8.25(\mathrm{~d}, J=8.5 \mathrm{~Hz}, 1 \mathrm{H}), 8.15$ (d, $J=8.5 \mathrm{~Hz}, 1 \mathrm{H}), 8.07(\mathrm{~d}, J=8.3 \mathrm{~Hz}, 1 \mathrm{H}), 7.87(\mathrm{t}, J=7.3 \mathrm{~Hz}, 1 \mathrm{H}), 7.67-7.75(\mathrm{~m}, 1 \mathrm{H}), 7.11(\mathrm{~d}$, $J=4.0 \mathrm{~Hz}, 2 \mathrm{H}), 7.01(\mathrm{dt}, J=8.2 \mathrm{~Hz}, J=4.2 \mathrm{~Hz}, 1 \mathrm{H}), 3.98(\mathrm{~s}, 3 \mathrm{H}) ;{ }^{13} \mathrm{C}-\mathrm{NMR}\left(\mathrm{DMSO}-d_{6}\right), \delta: 161.25$, $149.34,148.51,145.62,138.55,130.82,129.30,129.06,128.44,128.14,126.87,124.25,120.68$, 118.84, 118.27, 110.91, 56.05; HR-MS: for $\mathrm{C}_{17} \mathrm{H}_{15} \mathrm{~N}_{2} \mathrm{O}_{2}[\mathrm{M}+\mathrm{H}]^{+}$calculated $279.1134 \mathrm{~m} / \mathrm{z}$, found $279.1148 \mathrm{~m} / \mathrm{z}$.

N-(3-Methoxyphenyl)quinoline-2-carboxamide (13b). Yield 47\%; Mp. 117-118 ${ }^{\circ} \mathrm{C}$; IR (Zn/Se ATR, $\mathrm{cm}^{-1}$ ): 3,352w, 1,687m, 1,589m, 1,524m, 1,503m, 1,456m, 1,425 m, 1,334w, 1,284m, 1,203 m, 1,157m, $1,128 m, 1,049 s, 906 w, 876 m, 854 m, 823 w, 798 w, 762 s, 740 s, 685 m ;{ }^{1} \mathrm{H}-\mathrm{NMR}$ (DMSO-d $), \delta: 10.73$ (bs, $1 \mathrm{H}), 8.58(\mathrm{~d}, J=8.5 \mathrm{~Hz}, 1 \mathrm{H}), 8.19-8.32(\mathrm{~m}, 2 \mathrm{H}), 8.07(\mathrm{~d}, J=8.0 \mathrm{~Hz}, 1 \mathrm{H}), 7.82-7.96(\mathrm{~m}, 1 \mathrm{H})$, $7.65-7.79$ (m, 2H), 7.59 (dd, $J=8.0 \mathrm{~Hz}, J=1.0 \mathrm{~Hz}, 1 \mathrm{H}), 7.29$ (t, $J=8.2 \mathrm{~Hz}, 1 \mathrm{H}), 6.72$ (dd, $J=8.3 \mathrm{~Hz}$, $J=2.01 \mathrm{~Hz}, 1 \mathrm{H}), 3.78(\mathrm{~s}, 3 \mathrm{H}) ;{ }^{13} \mathrm{C}-\mathrm{NMR}$ (DMSO- $\left.d_{6}\right), \delta: 162.70,159.61,149.99,145.88,139.53$, 138.23, 130.67, 129.61, 129.37, 128.97, 128.37, 128.16, 118.75, 112.47, 109.68, 105.91, 55.09; HR-MS: for $\mathrm{C}_{17} \mathrm{H}_{15} \mathrm{~N}_{2} \mathrm{O}_{2}[\mathrm{M}+\mathrm{H}]^{+}$calculated $279.1134 \mathrm{~m} / \mathrm{z}$, found $279.1129 \mathrm{~m} / \mathrm{z}$.

$\mathrm{N}$-(4-Methoxyphenyl)quinoline-2-carboxamide (13c) [48]. Yield 53\%; Mp. 130-131 ${ }^{\circ} \mathrm{C}$; ${ }^{1} \mathrm{H}-\mathrm{NMR}$ (DMSO- $d_{6}$ ), $\delta: 10.65$ (bs, $\left.1 \mathrm{H}\right), 8.57$ (d, $\left.J=8.5 \mathrm{~Hz}, 1 \mathrm{H}\right), 8.24$ (d, $\left.J=8.5 \mathrm{~Hz}, 2 \mathrm{H}\right), 8.07$ (d, $J=7.8 \mathrm{~Hz}$, 1H), 7.82-7.95 (m, 3H), 7.63-7.78 (m, 1H), $6.97(\mathrm{~d}, J=9.0 \mathrm{~Hz}, 2 \mathrm{H}), 3.75(\mathrm{~s}, 3 \mathrm{H}) ;{ }^{13} \mathrm{C}-\mathrm{NMR}$ (DMSO- $d_{6}$ ), $\delta$ : 162.28, 155.80, 150.25, 145.90, 138.09, 131.47, 130.59, 129.32, 128.87, 128.22, 128.11, 121.85, 118.73, 113.87, 55.17; HR-MS: for $\mathrm{C}_{17} \mathrm{H}_{15} \mathrm{~N}_{2} \mathrm{O}_{2}[\mathrm{M}+\mathrm{H}]^{+}$calculated $279.1134 \mathrm{~m} / z$, found $279.1145 \mathrm{~m} / \mathrm{z}$.

N-(2-Methylphenyl)quinoline-2-carboxamide (14a). Yield 40\%; Mp. 100-101 ${ }^{\circ} \mathrm{C}$; IR (Zn/Se ATR, $\mathrm{cm}^{-1}$ ): 3,334w, 1,686s, 1,587s, 1,528s, 1,498m, 1,454s, 1,427s, 1,422 m, 1,373w, 1,305m, 1,249w, $1,201 w, 1,132 m, 1,091 w, 1,040 w, 1,013 w, 981 w, 954 m, 932 w, 907 m, 872 m, 842 s, 793 w, 765 s, 750 s$, $731 s, 681 s ;{ }^{1} \mathrm{H}-\mathrm{NMR}\left(\mathrm{DMSO}-d_{6}\right), \delta: 10.45(\mathrm{bs}, 1 \mathrm{H}), 8.60(\mathrm{~d}, J=8.5 \mathrm{~Hz}, 1 \mathrm{H}), 8.24(\mathrm{~d}, J=8.5 \mathrm{~Hz}, 1 \mathrm{H})$, $8.17(\mathrm{~d}, J=8.3 \mathrm{~Hz}, 1 \mathrm{H}), 8.08(\mathrm{~d}, J=8.0 \mathrm{~Hz}, 1 \mathrm{H}), 7.95(\mathrm{~d}, J=7.8 \mathrm{~Hz}, 1 \mathrm{H}), 7.83-7.91(\mathrm{~m}, 1 \mathrm{H})$, $7.69-7.77(\mathrm{~m}, 1 \mathrm{H}), 7.22-7.31(\mathrm{~m}, 2 \mathrm{H}), 7.08-7.16(\mathrm{~m}, 1 \mathrm{H}), 2.37(\mathrm{~s}, 3 \mathrm{H}) ;{ }^{13} \mathrm{C}-\mathrm{NMR}$ (DMSO- $\left.d_{6}\right)$, $\delta: 161.92,149.67,145.74,138.35,135.97,130.72,130.42,130.01,129.38,129.01,128.38,128.11$, 126.40, 124.96, 122.65, 118.49, 17.49; HR-MS: for $\mathrm{C}_{17} \mathrm{H}_{15} \mathrm{~N}_{2} \mathrm{O}[\mathrm{M}+\mathrm{H}]^{+}$calculated $263.1184 \mathrm{~m} / z$, found $263.1182 \mathrm{~m} / \mathrm{z}$.

N-(3-Methylphenyl)quinoline-2-carboxamide (14b). Yield 43\%; Mp. 82-83 ${ }^{\circ} \mathrm{C}$; IR (Zn/Se ATR, $\left.\mathrm{cm}^{-1}\right): 3,355 w, 1,685 m, 1,592 \mathrm{~m}, 1,527 s, 1,503 s 1,457 w, 1,424 \mathrm{~m}, 1,300 w, 1,171 w, 1,125 m, 908 w$, $852 m, 773 s, 740 w, 690 s$; ${ }^{1} \mathrm{H}-\mathrm{NMR}$ (DMSO- $d_{6}$ ), $\delta$ : 10.66 (bs, $\left.1 \mathrm{H}\right), 8.61(\mathrm{~d}, J=8.5 \mathrm{~Hz}, 1 \mathrm{H}), 8.25$ (dd, $J=7.9 \mathrm{~Hz}, J=5.40 \mathrm{~Hz}, 2 \mathrm{H}), 8.10(\mathrm{~d}, J=8.0 \mathrm{~Hz}, 1 \mathrm{H}), 7.90(\mathrm{t}, J=7.5 \mathrm{~Hz}, 1 \mathrm{H}), 7.67-7.84(\mathrm{~m}, 3 \mathrm{H})$, 
$7.27(\mathrm{t}, J=7.7 \mathrm{~Hz}, 1 \mathrm{H}), 6.96(\mathrm{~d}, J=7.3 \mathrm{~Hz}, 1 \mathrm{H}), 2.32(\mathrm{~s}, 3 \mathrm{H}) ;{ }^{13} \mathrm{C}-\mathrm{NMR}$ (DMSO- $\left.d_{6}\right), \delta: 162.54$, $150.03,145.88,138.22,138.20,138.03,130.68,129.35,128.94,128.65,128.35,128.15$, 124.75, 120.72, 118.70, 117.35, 21.23; HR-MS: for $\mathrm{C}_{17} \mathrm{H}_{15} \mathrm{~N}_{2} \mathrm{O}[\mathrm{M}+\mathrm{H}]^{+}$calculated $263.1184 \mathrm{~m} / z$, found $263.1191 \mathrm{~m} / \mathrm{z}$.

$\mathrm{N}$-(4-Methylphenyl)quinoline-2-carboxamide (14c). Yield 44\%; Mp. $107-108{ }^{\circ} \mathrm{C}$ (Mp. $109.5-110{ }^{\circ} \mathrm{C}$ [39]); ${ }^{1} \mathrm{H}-\mathrm{NMR}$ (DMSO- $d_{6}$ ), $\delta: 10.67$ (bs, $\left.1 \mathrm{H}\right), 8.59$ (d, $\left.J=8.5 \mathrm{~Hz}, 1 \mathrm{H}\right), 8.18-8.30(\mathrm{~m}, 2 \mathrm{H}), 8.08$ (d, $J=8.0 \mathrm{~Hz}, 1 \mathrm{H}), 7.79-7.94(\mathrm{~m}, 3 \mathrm{H}), 7.72(\mathrm{t}, J=7.4 \mathrm{~Hz}, 1 \mathrm{H}), 7.18(\mathrm{~d}, J=8.3 \mathrm{~Hz}, 2 \mathrm{H}), 2.27$ (s, 3H); ${ }^{13} \mathrm{C}-\mathrm{NMR}$ (DMSO- $d_{6}$ ), $\delta: 162.51,150.17,145.93,138.21,135.86,133.09,130.69,129.39,129.24,128.94$, 128.35, 128.18, 120.27, 118.77, 20.59; HR-MS: for $\mathrm{C}_{17} \mathrm{H}_{15} \mathrm{~N}_{2} \mathrm{O}[\mathrm{M}+\mathrm{H}]^{+}$calculated $263.1184 \mathrm{~m} / z$, found $263.1193 \mathrm{~m} / \mathrm{z}$.

$\mathrm{N}$-(2-Fluorophenyl)quinoline-2-carboxamide (15a). Yield 30\%; Mp. $116-117{ }^{\circ} \mathrm{C}$; IR (Zn/Se ATR, $\mathrm{cm}^{-1}$ ): $3,328 w, 1,691 m, 1,615 m, 1,591 w, 1,530 s, 1,504 m, 1,477 w, 1,454 m, 1,428 m, 1,317 w, 1,247 w$, $1,185 w, 1,126 m, 1,088 w, 910 w, 837 m, 772 s, 746 s, 683 m ;{ }^{1} \mathrm{H}-\mathrm{NMR}$ (DMSO-d $), \delta: 10.48$ (bs, 1H), $8.57(\mathrm{~d}, J=8.5 \mathrm{~Hz}, 1 \mathrm{H}), 8.17-8.25(\mathrm{~m}, 2 \mathrm{H}), 8.13(\mathrm{~d}, J=8.5 \mathrm{~Hz}, 1 \mathrm{H}), 8.05(\mathrm{~d}, J=8.0 \mathrm{~Hz}, 1 \mathrm{H}), 7.85(\mathrm{t}$, $J=7.3 \mathrm{~Hz}, 1 \mathrm{H}), 7.65-7.76(\mathrm{~m}, 1 \mathrm{H}), 7.28-7.40(\mathrm{~m}, 1 \mathrm{H}), 7.13-7.27(\mathrm{~m}, 2 \mathrm{H}) ;{ }^{13} \mathrm{C}-\mathrm{NMR}$ (DMSO- $\left.d_{6}\right)$, $\delta: 162.00,153.58\left(\mathrm{~d},{ }^{1} J_{\mathrm{FC}}=244 \mathrm{~Hz}\right), 148.95,145.67,138.39,130.76,129.24,129.15(\mathrm{~d}$, $\left.{ }^{2} J_{\mathrm{FC}}=19.1 \mathrm{~Hz}\right), 128.45,128.08,125.70\left(\mathrm{~d},{ }^{3} J_{\mathrm{FC}}=11.0 \mathrm{~Hz}\right), 125.53\left(\mathrm{~d},{ }^{3} J_{\mathrm{FC}}=7.3 \mathrm{~Hz}\right), 124.63(\mathrm{~d}$, $\left.{ }^{4} J_{\mathrm{FC}}=3.7 \mathrm{~Hz}\right), 122.91,118.37,115.43\left(\mathrm{~d},{ }^{2} J_{\mathrm{FC}}=19.1 \mathrm{~Hz}\right)$; HR-MS: for $\mathrm{C}_{16} \mathrm{H}_{12} \mathrm{FN}_{2} \mathrm{O}[\mathrm{M}+\mathrm{H}]^{+}$ calculated $267.0934 \mathrm{~m} / \mathrm{z}$, found $267.0950 \mathrm{~m} / \mathrm{z}$.

$N$-(3-Fluorophenyl)quinoline-2-carboxamide (15b). Yield 38\%; Mp. $126-127{ }^{\circ} \mathrm{C}$; IR (Zn/Se ATR, $\left.\mathrm{cm}^{-1}\right): 3,343 w, 1,690 \mathrm{~s}, 1,588 m, 1,531 s, 1,504 m, 1,481 s, 1,409 s, 1,170 m, 1,137 m, 899 m, 841 s, 791 m$, $768 s, 738 m, 682 s ;{ }^{1} \mathrm{H}-\mathrm{NMR}$ (DMSO- $d_{6}$ ), $\delta: 10.91$ (bs, $\left.1 \mathrm{H}\right), 8.58(\mathrm{~d}, J=8.5 \mathrm{~Hz}, 1 \mathrm{H}), 8.16-8.31$ (m, $2 \mathrm{H}), 8.08(\mathrm{~d}, J=8.3 \mathrm{~Hz}, 1 \mathrm{H}), 7.95(\mathrm{~d}, J=11.8 \mathrm{~Hz}, 1 \mathrm{H}), 7.86-7.92(\mathrm{~m}, 1 \mathrm{H}), 7.79(\mathrm{~d}, J=8.3 \mathrm{~Hz}, 1 \mathrm{H})$, $7.68-7.75(\mathrm{~m}, 1 \mathrm{H}), 7.35-7.49(\mathrm{~m}, 1 \mathrm{H}), 6.96(\mathrm{td}, J=8.4 \mathrm{~Hz}, J=2.0 \mathrm{~Hz}, 1 \mathrm{H}) ;{ }^{13} \mathrm{C}-\mathrm{NMR}$ (DMSO- $\left.d_{6}\right)$, $\delta: 163.01,162.15\left(\mathrm{~d},{ }^{1} J_{\mathrm{FC}}=241 \mathrm{~Hz}\right), 149.73,145.86,140.11\left(\mathrm{~d},{ }^{3} J_{\mathrm{FC}}=11.0 \mathrm{~Hz}\right), 138.18,130.65$, $130.31\left(\mathrm{~d},{ }^{3} J_{\mathrm{FC}}=9.5 \mathrm{~Hz}\right), 129.33,128.97,128.39,128.12,118.77,116.15\left(\mathrm{~d},{ }^{4} J_{\mathrm{FC}}=2.9 \mathrm{~Hz}\right), 110.47(\mathrm{~d}$, $\left.{ }^{2} J_{\mathrm{FC}}=21.3 \mathrm{~Hz}\right), 107.09\left(\mathrm{~d},{ }^{2} J_{\mathrm{FC}}=26.4 \mathrm{~Hz}\right)$; HR-MS: for $\mathrm{C}_{16} \mathrm{H}_{12} \mathrm{FN}_{2} \mathrm{O}[\mathrm{M}+\mathrm{H}]^{+}$calculated $267.0934 \mathrm{~m} / z$, found $267.0953 \mathrm{~m} / \mathrm{z}$.

$\mathrm{N}$-(4-Fluorophenyl)quinoline-2-carboxamide (15c) [46,48]. Yield 33\%; Mp. $115-116{ }^{\circ} \mathrm{C}$; ${ }^{1} \mathrm{H}-\mathrm{NMR}$ (DMSO- $\left.d_{6}\right), \delta: 10.83$ (bs, $\left.1 \mathrm{H}\right), 8.57(\mathrm{~d}, J=8.3 \mathrm{~Hz}, 1 \mathrm{H}), 8.17-8.29(\mathrm{~m}, 2 \mathrm{H}), 8.06(\mathrm{~d}, J=8.0 \mathrm{~Hz}, 1 \mathrm{H})$, $7.94-8.02(\mathrm{~m}, 2 \mathrm{H}), 7.87(\mathrm{td}, J=7.7 \mathrm{~Hz}, J=1.3 \mathrm{~Hz}, 1 \mathrm{H}), 7.66-7.76(\mathrm{~m}, 1 \mathrm{H}), 7.17-7.28(\mathrm{~m}, 2 \mathrm{H})$; ${ }^{13} \mathrm{C}-\mathrm{NMR}\left(\mathrm{DMSO}-d_{6}\right), \delta: 162.76,158.58\left(\mathrm{~d},{ }^{1} J_{\mathrm{FC}}=237 \mathrm{~Hz}\right), 150.03,145.95,138.18,134.81(\mathrm{~d}$, $\left.{ }^{4} J_{\mathrm{FC}}=2.2 \mathrm{~Hz}\right), 130.67,129.39,128.98,128.37,128.17,122.31\left(\mathrm{~d},{ }^{3} J_{\mathrm{FC}}=7.3 \mathrm{~Hz}\right), 118.83,115.26(\mathrm{~d}$, ${ }^{2} J_{\mathrm{FC}}=22.7 \mathrm{~Hz}$ ); HR-MS: for $\mathrm{C}_{16} \mathrm{H}_{12} \mathrm{FN}_{2} \mathrm{O}[\mathrm{M}+\mathrm{H}]^{+}$calculated $267.0934 \mathrm{~m} / \mathrm{z}$, found $267.0954 \mathrm{~m} / \mathrm{z}$.

$\mathrm{N}$-(2-Chlorophenyl)quinoline-2-carboxamide (16a) [48]. Yield 39\%; Mp. $130-131{ }^{\circ} \mathrm{C}$; ${ }^{1} \mathrm{H}-\mathrm{NMR}$ (DMSO- $\left.d_{6}\right), \delta: 10.77$ (bs, $\left.1 \mathrm{H}\right), 8.58(\mathrm{~d}, J=8.5 \mathrm{~Hz}, 1 \mathrm{H}), 8.43(\mathrm{~d}, J=8.0 \mathrm{~Hz}, 1 \mathrm{H}), 8.21(\mathrm{~d}, J=8.5 \mathrm{~Hz}$, $1 \mathrm{H}), 8.10(\mathrm{~d}, J=8.5 \mathrm{~Hz}, 1 \mathrm{H}), 8.05(\mathrm{~d}, J=8.3 \mathrm{~Hz}, 1 \mathrm{H}), 7.85(\mathrm{t}, J=7.5 \mathrm{~Hz}, 1 \mathrm{H}), 7.64-7.75(\mathrm{~m}, 1 \mathrm{H})$, $7.54(\mathrm{~d}, J=7.8 \mathrm{~Hz}, 1 \mathrm{H}), 7.39(\mathrm{t}, J=7.7 \mathrm{~Hz}, 1 \mathrm{H}), 7.10-7.24(\mathrm{~m}, 1 \mathrm{H}),{ }^{13} \mathrm{C}-\mathrm{NMR}$ (DMSO- $\left.d_{6}\right), \delta: 161.54$, 
148.70, 145.47, 138.50, 134.21, 130.75, 129.29, 129.20, 129.07, 128.46, 128.00, 127.88, 125.23, 123.38, 121.27, 118.15; HR-MS: for $\mathrm{C}_{16} \mathrm{H}_{12} \mathrm{ClN}_{2} \mathrm{O}[\mathrm{M}+\mathrm{H}]^{+}$calculated $283.0638 \mathrm{~m} / z$, found $283.0652 \mathrm{~m} / \mathrm{z}$.

$\mathrm{N}$-(3-Chlorophenyl)quinoline-2-carboxamide (16b) [48]. Yield 46\%; Mp. $127-128{ }^{\circ} \mathrm{C}$; ${ }^{1} \mathrm{H}-\mathrm{NMR}$ (DMSO-d $\left.d_{6}\right), \delta: 10.90(\mathrm{bs}, 1 \mathrm{H}), 8.58(\mathrm{~d}, J=8.5 \mathrm{~Hz}, 1 \mathrm{H}), 8.18-8.31(\mathrm{~m}, 2 \mathrm{H}), 8.15(\mathrm{~s}, 1 \mathrm{H}), 8.07$ (d, $J=8.0 \mathrm{~Hz}, 1 \mathrm{H}), 7.82-7.97(\mathrm{~m}, 2 \mathrm{H}), 7.66-7.78(\mathrm{~m}, 1 \mathrm{H}), 7.40(\mathrm{t}, J=8.0 \mathrm{~Hz}, 1 \mathrm{H}), 7.11-7.23(\mathrm{~m}, 1 \mathrm{H})$; ${ }^{13} \mathrm{C}-\mathrm{NMR}$ (DMSO- $d_{6}$ ), $\delta: 163.02,149.67,145.87,139.85,138.20,133.12,130.68,130.36,129.34$, 128.98, 128.43, 128.14, 123.70, 119.82, 118.77; HR-MS: for $\mathrm{C}_{16} \mathrm{H}_{12} \mathrm{ClN}_{2} \mathrm{O}[\mathrm{M}+\mathrm{H}]^{+}$calculated $283.0638 \mathrm{~m} / \mathrm{z}$, found $283.0648 \mathrm{~m} / \mathrm{z}$.

$\mathrm{N}$-(4-Chlorophenyl)quinoline-2-carboxamide (16c). Yield 34\%; Mp. 134-135 ${ }^{\circ} \mathrm{C}$ (Mp. 135-135.5 ${ }^{\circ} \mathrm{C}$ [39]); ${ }^{1} \mathrm{H}-\mathrm{NMR}\left(\mathrm{DMSO}-d_{6}\right), \delta$ : $10.88(\mathrm{bs}, 1 \mathrm{H}), 8.58(\mathrm{~d}, J=8.5 \mathrm{~Hz}, 1 \mathrm{H}), 8.17-8.30(\mathrm{~m}, 2 \mathrm{H}), 8.08(\mathrm{~d}$, $J=8.0 \mathrm{~Hz}, 1 \mathrm{H}), 8.01(\mathrm{~d}, J=8.8 \mathrm{~Hz}, 2 \mathrm{H}), 7.84-7.93(\mathrm{~m}, 1 \mathrm{H}), 7.68-7.77(\mathrm{~m}, 1 \mathrm{H}), 7.43(\mathrm{~d}, J=8.8 \mathrm{~Hz}$, $2 \mathrm{H}$ ); ${ }^{13} \mathrm{C}-\mathrm{NMR}$ (DMSO- $d_{6}$ ), $\delta: 162.87,149.85,145.88,138.16,137.34,130.65,129.34,128.95$, 128.62, 128.37, 128.14, 127.69, 121.92, 118.78; HR-MS: for $\mathrm{C}_{16} \mathrm{H}_{12} \mathrm{ClN}_{2} \mathrm{O}[\mathrm{M}+\mathrm{H}]^{+}$calculated $283.0638 \mathrm{~m} / \mathrm{z}$, found $283.0631 \mathrm{~m} / \mathrm{z}$.

$\mathrm{N}$-(2-Bromophenyl)quinoline-2-carboxamide (17a). Yield 26\%; Mp. 134-135 ${ }^{\circ} \mathrm{C}$; IR (Zn/Se ATR, $\left.\mathrm{cm}^{-1}\right): 3,277 w, 1,689 \mathrm{~s}, 1,588 m, 1,579 m, 1,543 m, 1,530 s, 1,496 m, 1,440 m, 1,427 m, 1,302 w, 1,132 w$, 1,204m, 908w, 842m, 768s, 736m, 698m; ${ }^{1} \mathrm{H}-\mathrm{NMR}$ (DMSO- $d_{6}$ ), $\delta: 10.82$ (bs, $\left.1 \mathrm{H}\right), 8.60(\mathrm{~d}, J=8.5 \mathrm{~Hz}$, $1 \mathrm{H}), 8.44(\mathrm{~d}, J=8.3 \mathrm{~Hz}, 1 \mathrm{H}), 8.23(\mathrm{~d}, J=8.5 \mathrm{~Hz}, 1 \mathrm{H}), 8.13(\mathrm{~d}, J=8.5 \mathrm{~Hz}, 1 \mathrm{H}), 8.07(\mathrm{~d}, J=8.3 \mathrm{~Hz}$, $1 \mathrm{H}), 7.87(\mathrm{t}, J=7.5 \mathrm{~Hz}, 1 \mathrm{H}), 7.64-7.77(\mathrm{~m}, 2 \mathrm{H}), 7.44(\mathrm{t}, J=7.8 \mathrm{~Hz}, 1 \mathrm{H}), 7.10(\mathrm{t}, J=7.7 \mathrm{~Hz}, 1 \mathrm{H})$; ${ }^{13} \mathrm{C}-\mathrm{NMR}$ (DMSO- $d_{6}$ ), $\delta: 161.61,148.71,145.50,138.60,135.46,132.58,130.83,129.26,129.13$, 128.55, 128.53, 128.08, 125.74, 121.39, 118.19, 114.08; HR-MS: for $\mathrm{C}_{16} \mathrm{H}_{12} \mathrm{BrN}_{2} \mathrm{O}[\mathrm{M}+\mathrm{H}]^{+}$calculated $327.0133 \mathrm{~m} / \mathrm{z}$, found $327.0138 \mathrm{~m} / \mathrm{z}$.

$\mathrm{N}$-(3-Bromophenyl)quinoline-2-carboxamide (17b). Yield 35\%; Mp. 139-140 ${ }^{\circ} \mathrm{C}$; IR (Zn/Se ATR, $\left.\mathrm{cm}^{-1}\right): 3,318 w, 1,687 m, 1,581 m, 1,519 m, 1,478 w, 1,408 m, 1,296 w, 1,124 m, 1,067 w, 912 w, 847 m, 764 s$, $685 \mathrm{~m} ;{ }^{1} \mathrm{H}-\mathrm{NMR}$ (DMSO-d $\left.d_{6}\right), \delta: 10.89(\mathrm{bs}, 1 \mathrm{H}), 8.60(\mathrm{~d}, J=8.3 \mathrm{~Hz}, 1 \mathrm{H}), 8.19-8.32(\mathrm{~m}, 3 \mathrm{H}), 8.09$ (d, $J=8.0 \mathrm{~Hz}, 1 \mathrm{H}), 7.96(\mathrm{~d}, J=7.5 \mathrm{~Hz}, 1 \mathrm{H}), 7.87-7.93(\mathrm{~m}, 1 \mathrm{H}), 7.68-7.78(\mathrm{~m}, 1 \mathrm{H}), 7.27-7.40(\mathrm{~m}, 2 \mathrm{H})$; ${ }^{13} \mathrm{C}-\mathrm{NMR}$ (DMSO- $d_{6}$ ), $\delta: 163.02,149.67,145.86,139.98,138.21,130.69,130.67,129.32,128.97$, 128.44, 128.14, 126.59, 122.66, 121.55, 119.14, 118.77; HR-MS: for $\mathrm{C}_{16} \mathrm{H}_{12} \mathrm{BrN}_{2} \mathrm{O}[\mathrm{M}+\mathrm{H}]^{+}$calculated $327.0133 \mathrm{~m} / \mathrm{z}$, found $327.0143 \mathrm{~m} / \mathrm{z}$.

$N$-(4-Bromophenyl)quinoline-2-carboxamide (17c). Yield 57\%; Mp. 157-158 ${ }^{\circ} \mathrm{C}$; IR (Zn/Se ATR, $\left.\mathrm{cm}^{-1}\right): 3,355 w, 1,693 s, 1,581 m, 1,522 s, 1,496 s, 1,423 w, 1,389 m, 1,305 w, 1,120 m, 1,095 w, 1,068 m$, 998w, 907w, 839s, 807s, 769s, 693w; ${ }^{1} \mathrm{H}-\mathrm{NMR}$ (DMSO- $d_{6}$ ), $\delta: 10.84$ (bs, $1 \mathrm{H}$ ), 8.58 (d, $J=8.5 \mathrm{~Hz}$, $1 \mathrm{H}), 8.18-8.30(\mathrm{~m}, 2 \mathrm{H}), 8.07(\mathrm{~d}, J=8.3 \mathrm{~Hz}, 1 \mathrm{H}), 7.95(\mathrm{~d}, J=8.8 \mathrm{~Hz}, 2 \mathrm{H}), 7.86-7.92(\mathrm{~m}, 1 \mathrm{H})$, $7.67-7.78(\mathrm{~m}, 1 \mathrm{H}), 7.56(\mathrm{~d}, J=8.8 \mathrm{~Hz}, 2 \mathrm{H}) ;{ }^{13} \mathrm{C}-\mathrm{NMR}$ (DMSO- $\left.d_{6}\right), \delta: 162.86,149.82,145.87,138.16$, 137.74, 131.53, 130.64, 129.33, 128.94, 128.37, 128.12, 122.28, 118.77, 115.80; HR-MS: for $\mathrm{C}_{16} \mathrm{H}_{12} \mathrm{BrN}_{2} \mathrm{O}[\mathrm{M}+\mathrm{H}]^{+}$calculated $327.0133 \mathrm{~m} / \mathrm{z}$, found $327.0129 \mathrm{~m} / \mathrm{z}$. 
$N$-(2-Trifluoromethylphenyl)quinoline-2-carboxamide (18a). Yield 32\%; Mp. 120-121 ${ }^{\circ} \mathrm{C}$; IR (Zn/Se ATR, $\left.\mathrm{cm}^{-1}\right): 3,316 w, 1,698 s, 1,590 s, 1,537 s, 1,498 w, 1,452 m, 1,423 m, 1,320 m, 1,288 m, 1,244 w$, $1,202 w, 1,165 m, 1,124 m, 1,094 m, 1,054 m, 1,026 m, 953 w, 906 w, 871 w, 836 m, 792 w, 763 s, 676 m$; ${ }^{1} \mathrm{H}-\mathrm{NMR}\left(\mathrm{DMSO}-d_{6}\right), \delta: 10.78(\mathrm{bs}, 1 \mathrm{H}), 8.61(\mathrm{~d}, J=8.3 \mathrm{~Hz}, 1 \mathrm{H}), 8.36(\mathrm{~d}, J=8.3 \mathrm{~Hz}, 1 \mathrm{H}), 8.23(\mathrm{~d}$, $J=8.3 \mathrm{~Hz}, 1 \mathrm{H}), 8.07(\mathrm{t}, J=8.3 \mathrm{~Hz}, 2 \mathrm{H}), 7.87(\mathrm{t}, J=7.5 \mathrm{~Hz}, 1 \mathrm{H}), 7.64-7.81(\mathrm{~m}, 3 \mathrm{H}), 7.38(\mathrm{t}$, $J=7.7 \mathrm{~Hz}, 1 \mathrm{H}) ;{ }^{13} \mathrm{C}-\mathrm{NMR}$ (DMSO- $\left.d_{6}\right), \delta: 162.05,148.48,145.53,138.74,135.14,133.57,131.01$, $129.48\left(\mathrm{q},{ }^{2} J_{\mathrm{FC}}=37 \mathrm{~Hz}\right), 129.21,129.17,128.68,128.16,126.41\left(\mathrm{q},{ }^{3} J_{\mathrm{FC}}=5.1 \mathrm{~Hz}\right), 125.05,124.10(\mathrm{q}$, $\left.{ }^{1} J_{\mathrm{FC}}=274 \mathrm{~Hz}\right), 123.89\left(\mathrm{q},{ }^{3} J_{\mathrm{FC}}=5.9 \mathrm{~Hz}\right), 118.31$; HR-MS: for $\mathrm{C}_{17} \mathrm{H}_{12} \mathrm{~F}_{3} \mathrm{~N}_{2} \mathrm{O}[\mathrm{M}+\mathrm{H}]^{+}$calculated $317.0896 \mathrm{~m} / \mathrm{z}$, found $317.0891 \mathrm{~m} / \mathrm{z}$.

$N$-(3-Trifluoromethylphenyl)quinoline-2-carboxamide (18b). Yield 31\%; Mp. 121-122 ${ }^{\circ} \mathrm{C}$; IR (Zn/Se ATR, $\left.\mathrm{cm}^{-1}\right): 3,339 w, 1,692 s, 1,614 w, 1,536 m, 1,490 m, 1,424 w, 1,330 s, 1,223 w, 1,166 m, 1,109 s$, $1,091 s, 1,065 m, 952 w, 933 w, 874 s, 844 m, 808 s, 771 s, 744 w, 698 s ;{ }^{1} \mathrm{H}-\mathrm{NMR}$ (DMSO- $d_{6}$ ), $\delta: 11.08$ (bs, $1 \mathrm{H}), 8.59(\mathrm{~d}, J=8.5 \mathrm{~Hz}, 1 \mathrm{H}), 8.46(\mathrm{~s}, 1 \mathrm{H}), 8.17-8.31(\mathrm{~m}, 3 \mathrm{H}), 8.08(\mathrm{~d}, J=8.0 \mathrm{~Hz}, 1 \mathrm{H}), 7.89$ (t, $J=7.4 \mathrm{~Hz}, 1 \mathrm{H}), 7.68-7.78(\mathrm{~m}, 1 \mathrm{H}), 7.61(\mathrm{t}, J=8.0 \mathrm{~Hz}, 1 \mathrm{H}), 7.46(\mathrm{~d}, J=7.5 \mathrm{~Hz}, 1 \mathrm{H}) ;{ }^{13} \mathrm{C}-\mathrm{NMR}$ $\left(\mathrm{DMSO}-d_{6}\right), \delta: 163.26,149.64,145.90,139.23,138.21,130.69,129.86,129.35\left(\mathrm{q},{ }^{2} J_{\mathrm{FC}}=32 \mathrm{~Hz}\right), 129.34$, $129.03,128.45,128.16,124.20\left(\mathrm{q},{ }^{1} J_{\mathrm{FC}}=273 \mathrm{~Hz}\right), 123.91,120.24\left(\mathrm{q},{ }^{3} J_{\mathrm{FC}}=3.7 \mathrm{~Hz}\right), 118.78,116.61$ (q, ${ }^{3} J_{\mathrm{FC}}=3.7 \mathrm{~Hz}$ ); HR-MS: for $\mathrm{C}_{17} \mathrm{H}_{12} \mathrm{~F}_{3} \mathrm{~N}_{2} \mathrm{O}[\mathrm{M}+\mathrm{H}]^{+}$calculated $317.0896 \mathrm{~m} / z$, found $317.0892 \mathrm{~m} / z$.

$N$-(4-Trifluoromethylphenyl)quinoline-2-carboxamide (18c) [41,44]. Yield 43\%; Mp. 147-148 ${ }^{\circ} \mathrm{C}$; ${ }^{1} \mathrm{H}-\mathrm{NMR}\left(\mathrm{DMSO}-d_{6}\right), \delta: 11.02$ (bs, $\left.1 \mathrm{H}\right), 8.59(\mathrm{~d}, J=8.3 \mathrm{~Hz}, 1 \mathrm{H}), 8.26(\mathrm{~d}, J=8.5 \mathrm{~Hz}, 1 \mathrm{H}), 8.23(\mathrm{~d}$, $J=8.3 \mathrm{~Hz}, 1 \mathrm{H}), 8.19(\mathrm{~d}, J=8.5 \mathrm{~Hz}, 2 \mathrm{H}), 8.08(\mathrm{~d}, J=8.0 \mathrm{~Hz}, 1 \mathrm{H}), 7.86-7.93(\mathrm{~m}, 1 \mathrm{H}), 7.69-7.77$ (m, $3 \mathrm{H}$ ); ${ }^{13} \mathrm{C}-\mathrm{NMR}$ (DMSO-d ${ }_{6}$ ), $\delta: 163.27,149.63,145.88,141.95,138.23,130.69,129.38,129.03$, $128.48,128.14,125.96\left(\mathrm{q},{ }^{3} J_{\mathrm{FC}}=3.7 \mathrm{~Hz}\right), 124.39\left(\mathrm{q},{ }^{1} J_{\mathrm{FC}}=271 \mathrm{~Hz}\right), 124.00\left(\mathrm{q},{ }^{2} J_{\mathrm{FC}}=32 \mathrm{~Hz}\right), 120.29$, 118.81; HR-MS: for $\mathrm{C}_{17} \mathrm{H}_{12} \mathrm{~F}_{3} \mathrm{~N}_{2} \mathrm{O}[\mathrm{M}+\mathrm{H}]^{+}$calculated $317.0896 \mathrm{~m} / \mathrm{z}$, found $317.0890 \mathrm{~m} / z$.

$\mathrm{N}$-(2-Nitrophenyl)quinoline-2-carboxamide (19a). Yield 29\%; Mp. 181-182 ${ }^{\circ} \mathrm{C}$ (Mp. 179.5-180 $\left.{ }^{\circ} \mathrm{C}[39]\right)$; ${ }^{1} \mathrm{H}-\mathrm{NMR}$ (DMSO-d $\left.d_{6}\right), \delta: 12.55(\mathrm{bs}, 1 \mathrm{H}), 8.75(\mathrm{~d}, J=8.5 \mathrm{~Hz}, 1 \mathrm{H}), 8.60(\mathrm{~d}, J=8.3 \mathrm{~Hz}, 1 \mathrm{H}), 8.17-8.28$ $(\mathrm{m}, 2 \mathrm{H}), 8.09(\mathrm{dd}, J=13.9,8.4 \mathrm{~Hz}, 2 \mathrm{H}), 7.86-7.95(\mathrm{~m}, 1 \mathrm{H}), 7.78-7.85(\mathrm{~m}, 1 \mathrm{H}), 7.70-7.77(\mathrm{~m}, 1 \mathrm{H})$, $7.34(\mathrm{t}, J=7.8 \mathrm{~Hz}, 1 \mathrm{H}) ;{ }^{13} \mathrm{C}-\mathrm{NMR}$ (DMSO- $\left.d_{6}\right), \delta: 162.61,148.50,145.57,138.55,137.44,135.73$, $133.47,130.94,129.20,129.16,128.70,128.09$, 125.80, 123.97, 121.77, 118.38; HR-MS: for $\mathrm{C}_{16} \mathrm{H}_{12} \mathrm{~N}_{3} \mathrm{O}_{3}[\mathrm{M}+\mathrm{H}]^{+}$calculated $294.0879 \mathrm{~m} / \mathrm{z}$, found $294.0888 \mathrm{~m} / \mathrm{z}$.

N-(3-Nitrophenyl)quinoline-2-carboxamide (19b). Yield 30\%; Mp. 189-190 ${ }^{\circ} \mathrm{C}$; IR (Zn/Se ATR, $\mathrm{cm}^{-1}$ ): 3,305w, 1,687m, 1,620w, 1,591w, 1,529m, 1,496w, 1,427m, 1,344m, 1,202w, 1,128m, 1,068w, 939w, 291m, 837m, 798m, 773s, 733s, 675s; ${ }^{1} \mathrm{H}-\mathrm{NMR}$ (DMSO-d $), \delta: 11.14$ (bs, 1H), 8.95 (t, $J=1.9 \mathrm{~Hz}, 1 \mathrm{H}), 8.57(\mathrm{~d}, J=8.5 \mathrm{~Hz}, 1 \mathrm{H}), 8.34(\mathrm{dd}, J=8.0 \mathrm{~Hz}, J=1.0 \mathrm{~Hz}, 1 \mathrm{H}), 8.16-8.28(\mathrm{~m}, 2 \mathrm{H})$, $8.06(\mathrm{~d}, J=8.0 \mathrm{~Hz}, 1 \mathrm{H}), 7.83-7.99(\mathrm{~m}, 2 \mathrm{H}), 7.69-7.77(\mathrm{~m}, 1 \mathrm{H}), 7.64(\mathrm{t}, J=8.2 \mathrm{~Hz}, 1 \mathrm{H}) ;{ }^{13} \mathrm{C}-\mathrm{NMR}$ (DMSO- $d_{6}$ ), $\delta$ : 163.35, 149.40, 147.88, 145.85, 139.56, 138.16, 130.64, 129.94, 129.30, 128.99, 128.44, 128.10, 126.34, 118.74, 118.34, 114.54; HR-MS: for $\mathrm{C}_{16} \mathrm{H}_{12} \mathrm{~N}_{3} \mathrm{O}_{3}[\mathrm{M}+\mathrm{H}]^{+}$calculated $294.0879 \mathrm{~m} / z$, found $294.0871 \mathrm{~m} / \mathrm{z}$. 
$\mathrm{N}$-(4-Nitrophenyl)quinoline-2-carboxamide (19c) [48,49]. Yield 29\%; Mp. 227-228 ${ }^{\circ} \mathrm{C}$; ${ }^{1} \mathrm{H}-\mathrm{NMR}$ (DMSO-d $)_{6}, \delta: 11.29$ (bs, 1H), $8.64(\mathrm{~d}, J=8.5 \mathrm{~Hz}, 1 \mathrm{H}), 8.18-8.35(\mathrm{~m}, 6 \mathrm{H}), 8.12(\mathrm{~d}, J=8.0 \mathrm{~Hz}, 1 \mathrm{H})$, $7.88-7.99(\mathrm{~m}, 1 \mathrm{H}), 7.78(\mathrm{~d}, J=7.8 \mathrm{~Hz}, 1 \mathrm{H}) ;{ }^{13} \mathrm{C}-\mathrm{NMR}$ (DMSO- $\left.d_{6}\right), \delta: 163.61,149.43,145.88,144.58$, $142.77,138.38,130.83,129.40,129.11,128.68,128.21,124.81,120.17,118.92$; HR-MS: for $\mathrm{C}_{16} \mathrm{H}_{12} \mathrm{~N}_{3} \mathrm{O}_{3}[\mathrm{M}+\mathrm{H}]^{+}$calculated $294.0879 \mathrm{~m} / \mathrm{z}$, found $294.0870 \mathrm{~m} / \mathrm{z}$.

\subsubsection{General Procedure for Synthesis of Carboxamide Derivatives 20-38c}

2-Naphthoic acid (1 g, $5.8 \mathrm{mmol})$ was suspended in dry toluene $(40 \mathrm{~mL})$ and $\mathrm{SOCl}_{2}(0.6 \mathrm{~mL}$, $8 \mathrm{mmol}, 1.4$ eq.) was added dropwise. The mixture was refluxed for $2 \mathrm{~h}$ and then evaporated to dryness. The crystalline residue was washed with dry toluene and used directly in the next step. Yield: 99\%; Mp. 46-47 ${ }^{\circ} \mathrm{C}$ (Mp. $43{ }^{\circ} \mathrm{C}$ [50]). Into the solution of 2-naphtoyl chloride (1.1 g, $\left.5.8 \mathrm{mmol}\right)$ in dry $\mathrm{CH}_{2} \mathrm{Cl}_{2}(30 \mathrm{~mL})$, triethylamine $(1.2 \mathrm{~mL}, 0.88 \mathrm{~g}, 8.66 \mathrm{mmol}, 1.5 \mathrm{eq}$.) and corresponding substituted amine $(5.8 \mathrm{mmol})$ were added dropwise. The mixture was stirred at room temperature for $20 \mathrm{~h}$ after which the solvent was removed under reduced pressure. The solid residue was washed with $10 \% \mathrm{HCl}$ and the crude product was recrystallized from $\mathrm{EtOH}$, cyclohexan, $\mathrm{CHCl}_{3}$ or acetone. The studied compounds 20-38c are presented in Table 2.

N-Isopropyl-2-naphthamide (20). Yield 18\%; Mp. $174-176{ }^{\circ} \mathrm{C}$ (Mp. $\left.170{ }^{\circ} \mathrm{C}[51]\right)$; IR (Zn/Se ATR, $\mathrm{cm}^{-1}$ ): 3,246m, 3,054w, 2,969w, 2,935w, 1,632m, 1,618s, 1,543s 1,499w, 1,352w, 1,327w, 1,294m, $1,201 w, 1,168 m, 1,136 w, 1,106 w, 953 w, 911 m, 835 m, 781 m$ 766w, 736m, 690w; ${ }^{1} \mathrm{H}-\mathrm{NMR}$ (DMSO- $d_{6}$ ), $\delta: 8.37-8.51(\mathrm{~m}, 2 \mathrm{H}), 7.88-8.09(\mathrm{~m}, 4 \mathrm{H}), 7.47-7.67(\mathrm{~m}, 2 \mathrm{H}), 4.18(\mathrm{dsxt}, J=13.8 \mathrm{~Hz}, J=6.7 \mathrm{~Hz}, 1 \mathrm{H})$, $1.21(\mathrm{~d}, J=6.5 \mathrm{~Hz}, 6 \mathrm{H}) ;{ }^{13} \mathrm{C}-\mathrm{NMR}$ (DMSO-d $\left.{ }_{6}\right), \delta: 165.42,134.05,132.23,132.16,128.79,127.72$, 127.60, 127.44, 127.33, 126.66, 124.38, 41.14, 22.41; HR-MS: for $\mathrm{C}_{14} \mathrm{H}_{16} \mathrm{NO}[\mathrm{M}+\mathrm{H}]^{+}$calculated $214.1226 \mathrm{~m} / \mathrm{z}$, found $214.1187 \mathrm{~m} / \mathrm{z}$.

N-Dodecyl-2-naphthamide (21). Yield 15\%; Mp. 102-103 ${ }^{\circ} \mathrm{C}$; IR (Zn/Se ATR, $\left.\mathrm{cm}^{-1}\right): 3,259 m, 2,915 s$, $2,848 s, 1,643 m, 1,619 s, 1,552 s, 1,501 w, 1,467 w, 1,432 w, 1,317 m, 1,204 w, 1,146 w, 954 w, 912 w, 871 w$, $839 w, 779 w, 734 w ;{ }^{1} \mathrm{H}-\mathrm{NMR}$ (DMSO- $\left.d_{6}\right), \delta: 8.61(\mathrm{t}, J=5.8 \mathrm{~Hz}, 1 \mathrm{H}), 8.37-8.52(\mathrm{~m}, 1 \mathrm{H}), 7.88-8.10$ (m, 4H), 7.46-7.69 (m, 2H), 3.26-3.34 (m, 2H), 1.55 (quin, $J=7.2 \mathrm{~Hz}, 2 \mathrm{H}), 1.13-1.37(\mathrm{~m}, 18 \mathrm{H})$, $0.78-0.87(\mathrm{~m}, 3 \mathrm{H}) ;{ }^{13} \mathrm{C}-\mathrm{NMR}$ (DMSO- $\left.d_{6}\right), \delta: 166.06,134.06,132.17,132.08,128.78,127.76,127.59$, 127.43, 127.28, 126.63, 124.20, 39.34, 31.32, 29.17, 29.16, 29.09, 29.06, 29.05, 28.84, 28.75, 26.55, 22.11, 13.95; HR-MS: for $\mathrm{C}_{23} \mathrm{H}_{34} \mathrm{NO}[\mathrm{M}+\mathrm{H}]^{+}$calculated $340.2635 \mathrm{~m} / \mathrm{z}$, found $340.2655 \mathrm{~m} / \mathrm{z}$.

1-(2-Naphthoyl)pyrrolidine (22). Yield 12\%; Mp. 76-77 ${ }^{\circ} \mathrm{C}\left(\mathrm{Mp} .75 .5-76.5{ }^{\circ} \mathrm{C}[52]\right)$; IR (Zn/Se ATR, $\left.\mathrm{cm}^{-1}\right): 3,050 m, 2,963 s, 2,863 s, 1,629 m, 1,608 s, 1,469 m, 1,434 w, 1,417 m, 1,353 w, 1,337 w ;{ }^{1} \mathrm{H}-\mathrm{NMR}$ $\left(\mathrm{DMSO}-d_{6}\right), \delta: 8.10(\mathrm{~s}, 1 \mathrm{H}), 7.97-8.03(\mathrm{~m}, 1 \mathrm{H}), 7.91-7.97(\mathrm{~m}, 2 \mathrm{H}), 7.51-7.64(\mathrm{~m}, 3 \mathrm{H}), 3.51(\mathrm{t}$, $J=6.9 \mathrm{~Hz}, 2 \mathrm{H}), 3.37-3.45(\mathrm{~m}, 2 \mathrm{H}), 1.82-1.92(\mathrm{~m}, 2 \mathrm{H}), 1.69-1.82(\mathrm{~m}, 2 \mathrm{H}) ;{ }^{13} \mathrm{C}-\mathrm{NMR}$ (DMSO- $\left.d_{6}\right)$, $\delta: 168.18,134.50,133.23,132.15,128.47,127.77,127.60,127.10,126.62,126.59,124.61,48.97$, 46.01, 25.99, 23.97; HR-MS: for $\mathrm{C}_{15} \mathrm{H}_{16} \mathrm{NO}[\mathrm{M}+\mathrm{H}]^{+}$calculated $226.1226 \mathrm{~m} / \mathrm{z}$, found $226.1200 \mathrm{~m} / z$.

1-(2-Naphthoyl)piperidine (23) [53]. Yield 40\%; Mp. 97-98 ${ }^{\circ} \mathrm{C}$; IR (Zn/Se ATR, $\left.\mathrm{cm}^{-1}\right): 3,050 w$, 2,933m, 2,852m, 1,633w, 1,612s, 1,476m, 1,432m, 1,347w, 1,278m, 1,269m, 1,229w, 1,199w, 1,126w, 
$1,100 w, 1,007 w, 954 w, 906 w, 833 w, 807 w, 758 m ;{ }^{1} \mathrm{H}-\mathrm{NMR}$ (DMSO-d $\left.)_{6}\right), \delta: 7.86-8.08(\mathrm{~m}, 4 \mathrm{H})$, 7.39-7.67 (m, 4H), 3.09-3.85 (m, 4H), 1.26-1.77 (m, 6H); ${ }^{13} \mathrm{C}-\mathrm{NMR}$ (DMSO- $\left.d_{6}\right), \delta: 168.84,133.91$, 132.96, 132.31, 128.27, 127.98, 127.65, 126.93, 126.66, 125.96, 124.29, 48.95, 43.22, 26.51, 26.01, 24.05; HR-MS: for $\mathrm{C}_{16} \mathrm{H}_{18} \mathrm{NO}[\mathrm{M}+\mathrm{H}]^{+}$calculated $240.1383 \mathrm{~m} / \mathrm{z}$, found $240.1349 \mathrm{~m} / z$.

N-Cyclopentyl-2-naphthamide (24). Yield 21\%; Mp. 190-191 ${ }^{\circ} \mathrm{C}$; IR (Zn/Se ATR, $\left.\mathrm{cm}^{-1}\right): 3,245 s$, 2,954m, 2,867w, 1,618m, 1,545m; ${ }^{1} \mathrm{H}-\mathrm{NMR}$ (DMSO-d $), \delta: 8.37-8.56(\mathrm{~m}, 2 \mathrm{H}), 7.89-8.11(\mathrm{~m}, 4 \mathrm{H})$, $7.48-7.67(\mathrm{~m}, 2 \mathrm{H}), 4.31$ (sxt, $J=7.0 \mathrm{~Hz}, 1 \mathrm{H}), 1.42-2.05(\mathrm{~m}, 8 \mathrm{H}) ;{ }^{13} \mathrm{C}-\mathrm{NMR}$ (DMSO- $d_{6}$ ), $\delta: 166.00$, 134.04, 132.20, 132.15, 128.78, 127.68, 127.60, 127.41, 127.38, 126.62, 124.45, 51.08, 32.20, 23.70; HR-MS: for $\mathrm{C}_{16} \mathrm{H}_{18} \mathrm{NO}[\mathrm{M}+\mathrm{H}]^{+}$calculated $240.1383 \mathrm{~m} / \mathrm{z}$, found $240.1372 \mathrm{~m} / \mathrm{z}$.

N-Cyclohexyl-2-naphthamide (25). Yield 45\%; Mp. 184-185 ${ }^{\circ} \mathrm{C}$ (Mp. 183-184 ${ }^{\circ} \mathrm{C}$ [52]); IR (Zn/Se ATR, $\left.\mathrm{cm}^{-1}\right): 3,316 m, 3,051 w, 2,933 s, 2,917 m, 2,850 m, 1,635 m, 1,622 s, 1,601 m, 1,531 s, 1,446 w$, $1,318 m, 1,288 m, 1,231 m, 1,202 w, 1,151 w, 1,081 m, 890 m, 859 w, 819 m, 779 m, 758 m, 728 w, 667 w ;$ ${ }^{1} \mathrm{H}-\mathrm{NMR}$ (DMSO- $d_{6}$ ), $\delta:$ 8.30-8.54 (m, 2H), 7.85-8.12 (m, 4H), 7.46-7.71 (m, 2H), 3.70-3.97 (m, $1 \mathrm{H}), 1.87(\mathrm{~d}, J=9.8 \mathrm{~Hz}, 2 \mathrm{H}), 1.74(\mathrm{dd}, J=9.8 \mathrm{~Hz}, J=2.5 \mathrm{~Hz}, 2 \mathrm{H}), 1.61(\mathrm{~d}, J=12.6 \mathrm{~Hz}, 1 \mathrm{H})$, $1.23-1.43(\mathrm{~m}, 4 \mathrm{H}), 1.04-1.21(\mathrm{~m}, 1 \mathrm{H}) ;{ }^{13} \mathrm{C}-\mathrm{NMR}$ (DMSO- $\left.d_{6}\right), \delta: 165.42,134.04,132.26,132.15$, $128.78,127.68,127.59,127.41,127.34,126.63,124.42,48.49$, 32.50, 25.31, 25.00; HR-MS: for $\mathrm{C}_{17} \mathrm{H}_{20} \mathrm{NO}[\mathrm{M}+\mathrm{H}]^{+}$calculated $254.1539 \mathrm{~m} / \mathrm{z}$, found $254.1493 \mathrm{~m} / \mathrm{z}$.

N-Cycloheptyl-2-naphthamide (26). Yield 18\%; Mp. $157-158{ }^{\circ} \mathrm{C}$; IR $\left(\mathrm{Zn} / \mathrm{Se}\right.$ ATR, $\left.\mathrm{cm}^{-1}\right): 3,318 m$, $2,918 m, 2,655 m, 1,635 m, 1,623 s, 1,600 w, 1,529 s, 1,504 m, 1,446 w, 1,314 m, 1,234 w, 1,201 w, 1,186 w$, $1,053 w, 907 w, 819 w, 777 w, 758 w, 729 w, 679 w ;{ }^{1} \mathrm{H}-\mathrm{NMR}$ (DMSO- $\left.d_{6}\right), \delta: 8.34-8.56$ (m, 2H), $7.87-8.12(\mathrm{~m}, 4 \mathrm{H}), 7.45-7.71(\mathrm{~m}, 2 \mathrm{H}), 4.04(\mathrm{qt}, J=8.8 \mathrm{~Hz}, J=4.6 \mathrm{~Hz}, 1 \mathrm{H}), 1.81-2.03(\mathrm{~m}, 2 \mathrm{H})$, $1.29-1.77(\mathrm{~m}, 10 \mathrm{H}) ;{ }^{13} \mathrm{C}-\mathrm{NMR}$ (DMSO- $\left.d_{6}\right), \delta: 165.20,134.05,132.35,132.17,128.78,127.69,127.61$, 127.41, 127.35, 126.63, 124.47, 50.59, 34.42, 27.89, 24.00; HR-MS: for $\mathrm{C}_{18} \mathrm{H}_{22} \mathrm{NO}[\mathrm{M}+\mathrm{H}]^{+}$calculated $268.1696 \mathrm{~m} / \mathrm{z}$, found $268.1621 \mathrm{~m} / \mathrm{z}$.

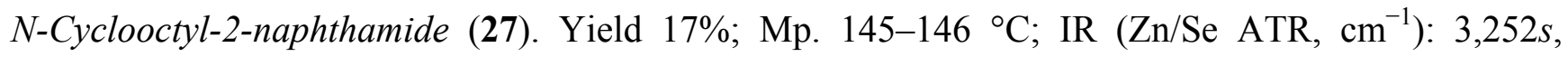
$2,921 s, 2,849 m, 1,634 s, 1,623 s, 1,548 s, 1,446 w, 1,348 w, 1,326 m, 1,279 w, 1,243 w, 1,199 w, 1,066 m$, $897 w, 864 w, 825 w, 774 m, 762 w, 737 m, 712 m ;{ }^{1} \mathrm{H}-\mathrm{NMR}$ (DMSO-d $), \delta: 8.30-8.55$ (m, 2H), 7.87-8.10 (m, 4H), 7.46-7.69 (m, 2H), 4.09 (qt, $J=8.3 \mathrm{~Hz}, J=4.2 \mathrm{~Hz}, 1 \mathrm{H}), 1.37-1.92(\mathrm{~m}, 14 \mathrm{H}) ;{ }^{13} \mathrm{C}-\mathrm{NMR}$ (DMSO- $d_{6}$ ), $\delta$ : 165.18, 134.03, 132.37, 132.15, 128.76, 127.66, 127.59, 127.38, 127.32, 126.60, 124.47, 49.39, 31.72, 26.86, 25.14, 23.60; HR-MS: for $\mathrm{C}_{19} \mathrm{H}_{24} \mathrm{NO}[\mathrm{M}+\mathrm{H}]^{+}$calculated $282.1852 \mathrm{~m} / z$, found $282.1873 \mathrm{~m} / \mathrm{z}$.

N-Phenyl-2-naphthamide (28). Yield 96\%; Mp. 175-176 ${ }^{\circ} \mathrm{C}\left(170{ }^{\circ} \mathrm{C}[50]\right.$, Mp. $\left.160{ }^{\circ} \mathrm{C}[54]\right) ; \mathrm{IR}(\mathrm{Zn} / \mathrm{Se}$ ATR, $\mathrm{cm}^{-1}$ ): 3,360m, 3,256w, 3,053w, 2,989w, 1,640s, 1,595s, 1,519s, 1,489s, 1,431s, 1,313m, 1,245w, $1,131 w, 1,076 w, 956 w, 912 m, 870 w, 824 w, 777 w, 749 s, 731 m, 689 m ;{ }^{1} \mathrm{H}-\mathrm{NMR}$ (DMSO-d $d_{6}$ ) [55,56], $\delta: 10.48(\mathrm{bs}, 1 \mathrm{H}), 8.61(\mathrm{~s}, 1 \mathrm{H}), 7.96-8.17(\mathrm{~m}, 4 \mathrm{H}), 7.88(\mathrm{~d}, J=8.0 \mathrm{~Hz}, 2 \mathrm{H}), 7.56-7.71(\mathrm{~m}, 2 \mathrm{H}), 7.39(\mathrm{t}$, $J=7.5 \mathrm{~Hz}, 2 \mathrm{H}), 7.05-7.19(\mathrm{~m}, 1 \mathrm{H}) ;{ }^{13} \mathrm{C}-\mathrm{NMR}$ (DMSO- $\left.d_{6}\right), \delta: 165.66,139.31,134.30,132.35,132.12$, 128.98, 128.69, 128.04, 128.03, 127.83, 127.71, 126.87, 124.53, 123.72, 120.42; HR-MS: for $\mathrm{C}_{17} \mathrm{H}_{14} \mathrm{NO}[\mathrm{M}+\mathrm{H}]^{+}$calculated $248.1070 \mathrm{~m} / z$, found $248.1095 \mathrm{~m} / \mathrm{z}$. 
N-Benzyl-2-naphthamide (29). Yield 42\%; Mp. 138-139 ${ }^{\circ} \mathrm{C}$ (Mp. 140-143 ${ }^{\circ} \mathrm{C}$ [57]); IR (Zn/Se ATR, $\left.\mathrm{cm}^{-1}\right): 3,287 s, 3,054 w, 3,027 w, 2,920 w, 1,635 s, 1,622 s, 1,600 w, 1,543 s, 1,504 m, 1,495 m, 1,449 w$, $1,414 m, 1,359 w, 1,316 m, 1,300 m, 1,263 m, 1,206 w, 1,146 w, 1,119 w, 1,047 w, 996 w, 910 m, 869 m$, $835 m, 777 m, 737 w, 719 m, 693 m ;{ }^{1} \mathrm{H}-\mathrm{NMR}$ (DMSO-d $), \delta: 9.25(\mathrm{t}, J=6.02 \mathrm{~Hz}, 1 \mathrm{H}), 8.53(\mathrm{~s}, 1 \mathrm{H})$, 7.91-8.11 (m, 4H), 7.53-7.70 (m, 2H), 7.29-7.46 (m, 4H), 7.21-7.28 (m, 1H), $4.56(\mathrm{~d}, J=6.02 \mathrm{~Hz}$, $2 \mathrm{H}) ;{ }^{13} \mathrm{C}-\mathrm{NMR}$ (DMSO- $\left.d_{6}\right), \delta: 166.29,139.70,134.17,132.18,131.72,128.87,128.32,127.92$, 127.63, 127.61, 127.55, 127.30, 126.77, 126.74, 124.22, 42.77; HR-MS: for $\mathrm{C}_{18} \mathrm{H}_{16} \mathrm{NO}[\mathrm{M}+\mathrm{H}]^{+}$calculated $262.1226 \mathrm{~m} / \mathrm{z}$, found $262.1257 \mathrm{~m} / \mathrm{z}$.

N-(2-Phenylethyl)-2-naphthamide (30). Yield 26\%; Mp. 133-134 ${ }^{\circ} \mathrm{C}$ (Mp. 133-134 ${ }^{\circ} \mathrm{C}$ [52]); IR (Zn/Se ATR, $\left.\mathrm{cm}^{-1}\right): 3,325 s, 3,054 w, 3,027 w, 2,933 w, 2,900 w, 1,639 s, 1,626 m, 1,600 m, 1,541 s$, $1,452 m, 1,302 w, 1,211 w, 1,074 w, 829 w, 779 w, 756 w, 692 w ;{ }^{1} \mathrm{H}-\mathrm{NMR}$ (DMSO-d 6 ), $\delta: 8.78$ (t, $J=5.5 \mathrm{~Hz}, 1 \mathrm{H}), 8.45(\mathrm{~s}, 1 \mathrm{H}), 7.86-8.11(\mathrm{~m}, 4 \mathrm{H}), 7.48-7.71(\mathrm{~m}, 2 \mathrm{H}), 7.12-7.41(\mathrm{~m}, 5 \mathrm{H}), 3.49-3.66(\mathrm{~m}$, 2H), $2.91(\mathrm{t}, J=7.5 \mathrm{~Hz}, 2 \mathrm{H}) ;{ }^{13} \mathrm{C}-\mathrm{NMR}$ (DMSO- $\left.d_{6}\right), \delta: 166.23,139.58,134.11,132.17,131.99$, $128.84,128.71,128.38,127.87,127.63,127.54,127.37,126.73,126.13,124.17,41.06,35.19$; HR-MS: for $\mathrm{C}_{19} \mathrm{H}_{18} \mathrm{NO}[\mathrm{M}+\mathrm{H}]^{+}$calculated $276.1383 \mathrm{~m} / \mathrm{z}$, found $276.1353 \mathrm{~m} / \mathrm{z}$.

N-(3-Hydroxyphenyl)-2-naphthamide (31b). Yield 69\%; Mp. $194{ }^{\circ} \mathrm{C}$; IR (Zn/Se ATR, $\left.\mathrm{cm}^{-1}\right): 3,349 s$, $1,712 s, 1,663 s, 1,603 s, 1,530 s, 1,432 s, 1,277 s, 1,220 s, 1,192 s, 1,144 s, 1,081 s, 970 s, 913 s, 861 s, 820 s$, $772 s, 760 s, 679 s ;{ }^{1} \mathrm{H}-\mathrm{NMR}\left(\mathrm{DMSO}-d_{6}\right), \delta: 10.32(\mathrm{~s}, 1 \mathrm{H}), 8.57(\mathrm{~s}, 1 \mathrm{H}), 8.11-7.99(\mathrm{~m}, 5 \mathrm{H}), 7.69-7.60$ $(\mathrm{m}, 2 \mathrm{H}), 7.43(\mathrm{t}, J=1.8 \mathrm{~Hz}, 1 \mathrm{H}), 7.25-7.10(\mathrm{~m}, 2 \mathrm{H}), 6.54(\mathrm{dt}, J=7.7 \mathrm{~Hz}, J=2.2 \mathrm{~Hz}, 1 \mathrm{H}) ;{ }^{13} \mathrm{C}-\mathrm{NMR}$ (DMSO- $d_{6}$ ), $\delta$ : 165.36, 157.44, 140.13, 134.10, 132.34, 131.97, 129.04, 128.76, 127.75, 127.73, $127.58,127.50,126.62,124.35,111.09,110.74,107.51$; HR-MS: for $\mathrm{C}_{17} \mathrm{H}_{14} \mathrm{NO}_{2}[\mathrm{M}+\mathrm{H}]^{+}$calculated $264.1019 \mathrm{~m} / \mathrm{z}$, found $264.1012 \mathrm{~m} / \mathrm{z}$.

N-(4-Hydroxyphenyl)-2-naphthamide (31c). Yield 66\%; Mp. $250-255{ }^{\circ} \mathrm{C}$; IR (Zn/Se ATR, $\left.\mathrm{cm}^{-1}\right)$ : $3,376 s, 3,055 w, 1,731 s, 1,660 s, 1,520 s, 1,311 s, 1,267 s, 1,221 s, 1,202 s, 1,081 s, 953 s, 802 s, 775 s, 760 s$, $754 s ;{ }^{1} \mathrm{H}-\mathrm{NMR}$ (DMSO-d $), \delta: 10.58(\mathrm{~s}, 1 \mathrm{H}), 8.61(\mathrm{~s}, 1 \mathrm{H}), 8.14-7.99(\mathrm{~m}, 5 \mathrm{H}), 7.70-7.61(\mathrm{~m}, 2 \mathrm{H})$, 7.40-7.34 (m, 2H), 6.79-6.74 (m, 2H); ${ }^{13} \mathrm{C}-\mathrm{NMR}$ (DMSO- $\left.d_{6}\right), \delta: 165.53,153.65,146.38,134.21$, 132.04, 131.36, 128.84, 127.93, 127.90, 127.66, 127.57, 124.32, 121.91, 121.31, 114.91; HR-MS: for $\mathrm{C}_{17} \mathrm{H}_{14} \mathrm{NO}_{2}[\mathrm{M}+\mathrm{H}]^{+}$calculated $264.1019 \mathrm{~m} / \mathrm{z}$, found $264.1010 \mathrm{~m} / \mathrm{z}$.

N-(2-Methoxyphenyl)-2-naphthamide (32a). Yield 46\%; Mp. $82{ }^{\circ} \mathrm{C}$; IR $\left(\mathrm{Zn} / \mathrm{Se}\right.$ ATR, $\left.\mathrm{cm}^{-1}\right): 3,274 s$, $3,060 w, 2,997 w, 2,973 w, 1,647 s, 1,598 m, 1,535 s, 1,493 s, 1,460 s, 1,433 s, 1,336 s, 1,259 s, 1,026 m, 749 s$; ${ }^{1} \mathrm{H}-\mathrm{NMR}$ (DMSO-d $\left.d_{6}\right), \delta: 9.62(\mathrm{~s}, 1 \mathrm{H}), 8.62(\mathrm{~s}, 1 \mathrm{H}), 8.10-8.08(\mathrm{~m}, 1 \mathrm{H}), 8.04(\mathrm{~d}, J=1.0 \mathrm{~Hz}, 2 \mathrm{H}), 8.01$ $(\mathrm{dd}, J=8.2 \mathrm{~Hz}, J=1.1 \mathrm{~Hz}, 1 \mathrm{H}), 7.85(\mathrm{dd}, J=7.9 \mathrm{~Hz}, J=1.3 \mathrm{~Hz}, 1 \mathrm{H}), 7.66-7.60(\mathrm{~m}, 2 \mathrm{H}), 7.22-7.18$ (m, 1H), 7.12-7.10 (m, 1H), $7.00(\mathrm{td}, J=7.7 \mathrm{~Hz}, J=1.2 \mathrm{~Hz}, 1 \mathrm{H}), 3.86(\mathrm{~s}, 3 \mathrm{H}) ;{ }^{13} \mathrm{C}-\mathrm{NMR}$ (DMSO- $d_{6}$ ), $\delta: 165.08,151.52,134.30,132.16,131.84,129.02,128.12,127.92,127.84,127.66,126.89,126.83$, 125.76, 124.36, 124.24, 120.24, 111.41, 55.73; HR-MS: for $\mathrm{C}_{18} \mathrm{H}_{16} \mathrm{NO}_{2}[\mathrm{M}+\mathrm{H}]^{+}$calculated $278.1176 \mathrm{~m} / z$, found $278.1179 \mathrm{~m} / \mathrm{z}$.

$N$-(3-Methoxyphenyl)-2-naphthamide (32b). Yield 47\%; Mp. $142{ }^{\circ} \mathrm{C}$; IR (Zn/Se ATR, $\left.\mathrm{cm}^{-1}\right): 3,243 s$, $3,005 w, 1,640 s, 1,591 s, 1,530 s, 1,451 s, 1,429 s, 1,301 s, 1,282 s, 1,158 s, 1,049 s, 771 s ;{ }^{1} \mathrm{H}-\mathrm{NMR}$ 
(DMSO- $\left.d_{6}\right), \delta: 10.43(\mathrm{~s}, 1 \mathrm{H}), 8.59(\mathrm{~s}, 1 \mathrm{H}), 8.11-8.07(\mathrm{~m}, 1 \mathrm{H}), 8.05(\mathrm{~s}, 1 \mathrm{H}), 8.04(\mathrm{~d}, J=1.5 \mathrm{~Hz}, 1 \mathrm{H})$, $8.02-8.00(\mathrm{~m}, 1 \mathrm{H}), 7.66-7.60(\mathrm{~m}, 2 \mathrm{H}), 7.55(\mathrm{t}, J=2.3 \mathrm{~Hz}, 1 \mathrm{H}), 7.45(\mathrm{dd}, J=8.0 \mathrm{~Hz}, J=1.0 \mathrm{~Hz}, 1 \mathrm{H})$, $7.28(\mathrm{t}, J=8.2 \mathrm{~Hz}, 1 \mathrm{H}), 6.71(\mathrm{dd}, J=8.2 \mathrm{~Hz}, J=1.8 \mathrm{~Hz}, 1 \mathrm{H}) ; 3.78(\mathrm{~s}, 3 \mathrm{H}) ;{ }^{13} \mathrm{C}-\mathrm{NMR}$ (DMSO- $\left.d_{6}\right)$, $\delta: 165.44,159.38,140.30,134.13,132.16,131.95,129.20,128.75,127.81,127.75,127.61,127.49$, 126.64, 124.26, 112.51, 109.10, 106.06, 54.90; HR-MS: for $\mathrm{C}_{18} \mathrm{H}_{16} \mathrm{NO}_{2}[\mathrm{M}+\mathrm{H}]^{+}$calculated $278.1176 \mathrm{~m} / z$, found $278.1175 \mathrm{~m} / \mathrm{z}$.

N-(4-Methoxyphenyl)-2-naphthamide (32c). Yield 65\%; Mp. $182{ }^{\circ} \mathrm{C}$; IR (Zn/Se ATR, $\left.\mathrm{cm}^{-1}\right): 3,371 s$, $3,049 w, 2,949 w, 2,835 w, 1,656 s, 1,598 s, 1,527 s, 1,511 s, 1,463 s, 1,414 s, 1,314 s, 1,303 s, 1,220 s$, $1,182 s, 1,033 s, 820 s, 761 s ;{ }^{1} \mathrm{H}-\mathrm{NMR}\left(\mathrm{DMSO}_{-}\right), \delta: 10.34(\mathrm{~s}, 1 \mathrm{H}), 8.58(\mathrm{~s}, 1 \mathrm{H}), 8.08(\mathrm{~d}, J=8.8 \mathrm{~Hz}$, $1 \mathrm{H}), 8.04(\mathrm{~s}, 2 \mathrm{H}), 8.00(\mathrm{~d}, J=8.3 \mathrm{~Hz}, 1 \mathrm{H}), 7.75(\mathrm{~d}, J=8.3 \mathrm{~Hz}, 2 \mathrm{H}), 7.66-7.59(\mathrm{~m}, 2 \mathrm{H}), 6.69(\mathrm{~d}$, $J=8.3 \mathrm{~Hz}, 2 \mathrm{H}), 3.76(\mathrm{~s}, 3 \mathrm{H}) ;{ }^{13} \mathrm{C}-\mathrm{NMR}$ (DMSO- $\left.d_{6}\right), \delta: 165.15,155.57,134.21,132.39,132.33$, 132.12, 128.92, 127.97, 127.81, 127.72, 127.67, 126.81, 124.46, 121.99, 113.78, 55.18; HR-MS: for $\mathrm{C}_{18} \mathrm{H}_{16} \mathrm{NO}_{2}[\mathrm{M}+\mathrm{H}]^{+}$calculated $278.1176 \mathrm{~m} / \mathrm{z}$, found $278.1173 \mathrm{~m} / \mathrm{z}$.

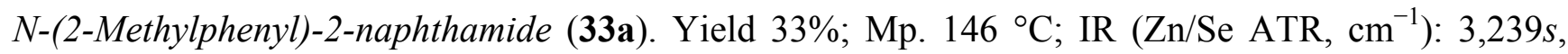
$3,057 w, 1,639 s, 1,584 s, 1,531 s, 1,454 s, 1,315 s, 1,134 s, 916 s, 777 s, 749 s, 731 s, 692 m$; ${ }^{1} \mathrm{H}-\mathrm{NMR}$ (DMSO- $\left.d_{6}\right), \delta: 10.09(\mathrm{~s}, 1 \mathrm{H}), 8.63(\mathrm{~s}, 1 \mathrm{H}), 8.10-8.08(\mathrm{~m}, 1 \mathrm{H}), 8.08-8.06(\mathrm{~m}, 2 \mathrm{H}), 8.04-8.01(\mathrm{~m}, 1 \mathrm{H})$, $7.67-7.60(\mathrm{~m}, 2 \mathrm{H}), 7.41(\mathrm{~d}, J=6.7 \mathrm{~Hz}, 1 \mathrm{H}), 7.30(\mathrm{~d}, J=7.3 \mathrm{~Hz}, 1 \mathrm{H}), 7.28(\mathrm{td}, J=7.5 \mathrm{~Hz}, J=1.5 \mathrm{~Hz}$, $1 \mathrm{H}), 7.19(\mathrm{td}, J=7.3 \mathrm{~Hz}, J=1.3 \mathrm{~Hz}, 1 \mathrm{H}) ; 2.29(\mathrm{~s}, 3 \mathrm{H}) ;{ }^{13} \mathrm{C}-\mathrm{NMR}$ (DMSO-d $), \delta: 166.05$, $137.16,134.95,134.41,132.82,132.55,131.02,129.62,128.69,128.68,128.42,128.33,127.49$, 127.27, 126.70, 126.67, 125.12, 18.66; HR-MS: for $\mathrm{C}_{18} \mathrm{H}_{16} \mathrm{NO}[\mathrm{M}+\mathrm{H}]^{+}$calculated $262.1226 \mathrm{~m} / z$, found $262.1225 \mathrm{~m} / \mathrm{z}$.

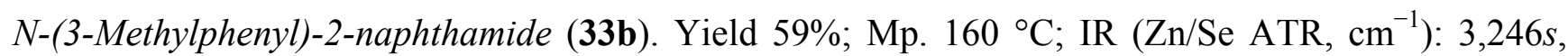
$3,055 w, 1,641 s, 1,590 s, 1,550 s, 1,432 s, 1,309 s, 1,130 s, 777 s, 749 s, 731 s, 690 s ;{ }^{1} \mathrm{H}-\mathrm{NMR}$ (DMSO- $d_{6}$ ), $\delta: 10.37(\mathrm{~s}, 1 \mathrm{H}), 8.59(\mathrm{~s}, 1 \mathrm{H}), 8.10-8.07(\mathrm{~m}, 1 \mathrm{H}), 8.04-8.02(\mathrm{~m}, 2 \mathrm{H}), 8.02-8.00(\mathrm{~m}, 1 \mathrm{H}), 7.69(\mathrm{~s}, 1 \mathrm{H})$, 7.66-7.60 (m, 3H), $7.26(\mathrm{t}, J=7.8 \mathrm{~Hz}, 1 \mathrm{H}), 6.94(\mathrm{~d}, J=7.3 \mathrm{~Hz}, 1 \mathrm{H}), 2.33(\mathrm{~s}, 3 \mathrm{H}) ;{ }^{13} \mathrm{C}-\mathrm{NMR}$ (DMSO- $\left.d_{6}\right)$, $\delta: 165.35,139.02,137.61,134.12,132.22,131.97,128.75,128.28,127.81,127.72,127.60,127.49$, 126.64, 124.29, 124.23, 120.84, 117.47, 21.03; HR-MS: for $\mathrm{C}_{18} \mathrm{H}_{16} \mathrm{NO}[\mathrm{M}+\mathrm{H}]^{+}$calculated $262.1226 \mathrm{~m} / z$, found $262.1224 \mathrm{~m} / \mathrm{z}$.

N-(4-Methylphenyl)-2-naphthamide (33c). Yield 67\%; Mp. $195{ }^{\circ} \mathrm{C}$ (Mp. $191{ }^{\circ} \mathrm{C}[50)$; IR (Zn/Se ATR, $\left.\mathrm{cm}^{-1}\right): 3,254 s, 3,056 w, 2,917 w, 1,640 s, 1,602 s, 1,538 s, 1,513 s, 1,404 s, 1,329 s, 1,132 s, 914 s, 811 s$, $754 s, 733 s, 707 m$; ${ }^{1} \mathrm{H}-\mathrm{NMR}$ (DMSO-d $)$ [56], $\delta: 10.38$ (s, 1H), 8.59 (s, 1H), 8.10-8.06 (m, 1H), 8.04 $(\mathrm{s}, 2 \mathrm{H}), 8.02-8.00(\mathrm{~m}, 1 \mathrm{H}), 7.73(\mathrm{~d}, J=8.5 \mathrm{~Hz}, 2 \mathrm{H}), 7.66-7.60(\mathrm{~m}, 2 \mathrm{H}), 7.18(\mathrm{~d}, J=8.3 \mathrm{~Hz}, 2 \mathrm{H}), 2.29$ $(\mathrm{s}, 3 \mathrm{H}) ;{ }^{13} \mathrm{C}-\mathrm{NMR}$ (DMSO-d $), \delta: 165.22,136.59,134.10,132.51,132.27,132.00,128.85,128.75$, $127.79,127.69,127.57,127.50,126.64,124.30,120.34,20.34$; HR-MS: for $\mathrm{C}_{18} \mathrm{H}_{16} \mathrm{NO}[\mathrm{M}+\mathrm{H}]^{+}$ calculated $262.1226 \mathrm{~m} / \mathrm{z}$, found $262.1225 \mathrm{~m} / \mathrm{z}$.

$N$-(2-Fluorophenyl)-2-naphthamide (34a). Yield 59\%; Mp. $122{ }^{\circ} \mathrm{C}$; IR (Zn/Se ATR, $\left.\mathrm{cm}^{-1}\right): 3,316 s$, $3,054 w, 3,023 w, 1,648 s, 1,541 s, 1,489 s, 1,456 s, 1,321 s, 1,257 s, 1,192 m, 829 m, 750 s ;{ }^{1} \mathrm{H}-\mathrm{NMR}$ $\left(\mathrm{DMSO}-d_{6}\right), \delta: 10.33(\mathrm{~s}, 1 \mathrm{H}), 8.64(\mathrm{~s}, 1 \mathrm{H}), 8.11-8.08(\mathrm{~m}, 1 \mathrm{H}), 8.06(\mathrm{~d}, J=1.0 \mathrm{~Hz}, 2 \mathrm{H}), 8.03-8.01$ (m, 
1H), 7.70-7.60 (m, 3H), 7.36-7.23 (m, 3H); ${ }^{13} \mathrm{C}-\mathrm{NMR} \quad\left(\mathrm{DMSO}-d_{6}\right), \delta: 165.38,155.64(\mathrm{~d}$, $J=245.8 \mathrm{~Hz}), 134.27,131.98,131.21,128.82,128.13,127.88,127.73,127.50,126.72(\mathrm{~d}, J=8.8 \mathrm{~Hz})$, 126.68, 126.68 (d, $J=7.6 \mathrm{~Hz}), 125.75$ (d, $J=11.4 \mathrm{~Hz}), 124.24,124.13$ (d, $J=3.8 \mathrm{~Hz}), 115.65$ (d, $J=20.5 \mathrm{~Hz}$ ); HR-MS: for $\mathrm{C}_{17} \mathrm{H}_{13} \mathrm{NOF}[\mathrm{M}+\mathrm{H}]^{+}$calculated $266.0976 \mathrm{~m} / \mathrm{z}$, found $266.0974 \mathrm{~m} / \mathrm{z}$.

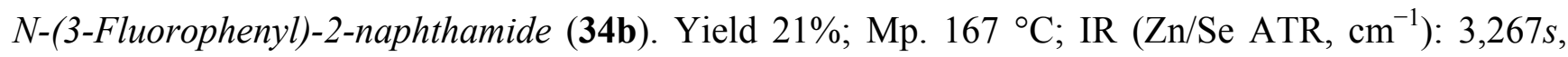
$3,140 w, 3,058 w, 1,642 s, 1,604 s, 1,541 s, 1,444 s, 1,322 s, 1,301 s, 1,142 m, 775 m, 765 m, 682 m$; ${ }^{1} \mathrm{H}-\mathrm{NMR}$ (DMSO- $\left.d_{6}\right), \delta: 10.64(\mathrm{~s}, 1 \mathrm{H}), 8.59(\mathrm{~s}, 1 \mathrm{H}), 8.11-8.09(\mathrm{~m}, 1 \mathrm{H}), 8.08-8.01(\mathrm{~m}, 3 \mathrm{H}), 7.83$ (dt, $J=11.8 \mathrm{~Hz}$, $J=2.5 \mathrm{~Hz}, 1 \mathrm{H}), 7.68-7.61(\mathrm{~m}, 3 \mathrm{H}), 7.42(\mathrm{dt}, J=8.2 \mathrm{~Hz}, J=7.0 \mathrm{~Hz}, 1 \mathrm{H}), 6.95$ (tdd, $J=8.5 \mathrm{~Hz}$, $J=2.5 \mathrm{~Hz}, J=0.75 \mathrm{~Hz}, 1 \mathrm{H}) ;{ }^{13} \mathrm{C}-\mathrm{NMR}$ (DMSO- $\left.d_{6}\right), \delta: 165.71,161.98(\mathrm{~d}, J=239.7 \mathrm{~Hz}), 140.89$ (d, $J=11.4 \mathrm{~Hz}), 134.23,131.93,131.83,130.06$ (d, $J=9.9 \mathrm{~Hz}), 128.79,127.93,127.90,127.75,127.53$, 126.73, 124.21, $115.90(\mathrm{~d}, J=3.1 \mathrm{~Hz}), 109.94(\mathrm{~d}, J=20.5 \mathrm{~Hz}), 106.90(\mathrm{~d}, J=25.8 \mathrm{~Hz})$; HR-MS: for $\mathrm{C}_{17} \mathrm{H}_{13} \mathrm{NOF}[\mathrm{M}+\mathrm{H}]^{+}$calculated $266.0976 \mathrm{~m} / z$, found $266.0972 \mathrm{~m} / \mathrm{z}$.

N-(4-Fluorophenyl)-2-naphthamide (34c). Yield 70\%; Mp. $191{ }^{\circ} \mathrm{C}$; IR (Zn/Se ATR, $\left.\mathrm{cm}^{-1}\right): 3,380 s$, $3,063 w, 1,654 s, 1,527 s, 1,506 s, 1,405 s, 1,313 m, 1,212 s, 1,197 s, 826 s, 814 s, 764 s ;{ }^{1} \mathrm{H}-\mathrm{NMR}$ $\left(\mathrm{DMSO}-d_{6}\right)$ [56], $\delta: 10.51(\mathrm{~s}, 1 \mathrm{H}), 8.58(\mathrm{~s}, 1 \mathrm{H}), 8.10-8.08(\mathrm{~m}, 1 \mathrm{H}), 8.07-8.00(\mathrm{~m}, 3 \mathrm{H}), 7.88-7.84(\mathrm{~m}$, 2H), 7.66-7.60 (m, 2H), 7.25-7.20 (m, 2H); ${ }^{13} \mathrm{C}-\mathrm{NMR}$ (DMSO-d $)_{6}, \delta: 165.36,158.22(\mathrm{~d}$, $J=238.9 \mathrm{~Hz}), 135.46$ (d, $J=2.3 \mathrm{~Hz}), 134.17,132.03,131.98,128.75,127.84,127.78,127.64,127.50$, 126.67, 124.24, $122.13(\mathrm{~d}, J=8.0 \mathrm{~Hz}), 115.01\left(\mathrm{~d}, J=22.0 \mathrm{~Hz}\right.$ ); HR-MS: for $\mathrm{C}_{17} \mathrm{H}_{13} \mathrm{NOF}[\mathrm{M}+\mathrm{H}]^{+}$ calculated $266.0976 \mathrm{~m} / \mathrm{z}$, found $266.0975 \mathrm{~m} / \mathrm{z}$.

$N$-(2-Chlorophenyl)-2-naphthamide (35a) [58]. Yield 45\%; Mp. $116{ }^{\circ} \mathrm{C}$; IR (Zn/Se ATR, $\left.\mathrm{cm}^{-1}\right)$ : $3,281 s, 3,061 w, 1,653 s, 1,583 s, 1,523 s, 1,439 s, 1,305 s, 1,053 m, 777 s, 759 s, 746 s ;{ }^{1} \mathrm{H}-\mathrm{NMR}$ (DMSO- $\left.d_{6}\right), \delta: 10.26(\mathrm{~s}, 1 \mathrm{H}), 8.65(\mathrm{~s}, 1 \mathrm{H}), 8.11-8.09(\mathrm{~m}, 1 \mathrm{H}), 8.09-8.05(\mathrm{~m}, 2 \mathrm{H}), 8.04-8.00(\mathrm{~m}, 1 \mathrm{H})$, $7.68-7.61(\mathrm{~m}, 3 \mathrm{H}), 7.59$ (dd, $J=7.9 \mathrm{~Hz}, J=1.3 \mathrm{~Hz}, 1 \mathrm{H}), 7.42(\mathrm{td}, J=7.7 \mathrm{~Hz}, J=1.5 \mathrm{~Hz}, 1 \mathrm{H}), 7.32$ $(\mathrm{td}, J=7.8 \mathrm{~Hz}, J=1.5 \mathrm{~Hz}, 1 \mathrm{H}) ;{ }^{13} \mathrm{C}-\mathrm{NMR}$ (DMSO- $d_{6}$ ), $\delta: 165.48,135.16,134.42,132.11,131.30$, $129.61,129.55,129.02,128.50,128.25,128.15,127.96,127.70,127.52,127.51,126.92,124.34$; HR-MS: for $\mathrm{C}_{17} \mathrm{H}_{13} \mathrm{NOCl}[\mathrm{M}+\mathrm{H}]^{+}$calculated $282.0680 \mathrm{~m} / \mathrm{z}$, found $282.0679 \mathrm{~m} / \mathrm{z}$.

$N$-(3-Chlorophenyl)-2-naphthamide (35b). Yield 67\%; Mp. $180{ }^{\circ} \mathrm{C}$; IR (Zn/Se ATR, $\left.\mathrm{cm}^{-1}\right): 3,262 s$, $3,236 s, 3,110 w, 3,055 w, 1,644 s, 1,591 s, 1,531 s, 1,420 s, 1,316 s, 1,301 s, 1,133 m, 778 s, 765 s, 695 m$; ${ }^{1} \mathrm{H}-\mathrm{NMR}\left(\mathrm{DMSO}-d_{6}\right), \delta: 10.61(\mathrm{~s}, 1 \mathrm{H}), 8.59(\mathrm{~s}, 1 \mathrm{H}), 8.11-8.09(\mathrm{~m}, 1 \mathrm{H}), 8.08-8.01(\mathrm{~m}, 4 \mathrm{H}), 7.78$ (ddd, $J=8.3 \mathrm{~Hz}, J=1.8 \mathrm{~Hz}, J=0.8 \mathrm{~Hz}, 1 \mathrm{H}), 7.68-7.61(\mathrm{~m}, 2 \mathrm{H}), 7.41(\mathrm{t}, J=8.2 \mathrm{~Hz}, 1 \mathrm{H}), 7.18$ (ddd, $J=8.0 \mathrm{~Hz}, J=1.9 \mathrm{~Hz}, J=0.8 \mathrm{~Hz}, 1 \mathrm{H}$ ); ${ }^{13} \mathrm{C}-\mathrm{NMR}$ (DMSO- $d_{6}$ ), $\delta: 165.69,140.61,134.24,132.86$, $131.93,131.77,130.14,128.81,127.95,127.92$, 127.78, 127.53, 126.75, 124.21, 123.21, 119.68, 118.55; HR-MS: for $\mathrm{C}_{17} \mathrm{H}_{13} \mathrm{NOCl}[\mathrm{M}+\mathrm{H}]^{+}$calculated $282.0680 \mathrm{~m} / \mathrm{z}$, found $282.0681 \mathrm{~m} / \mathrm{z}$.

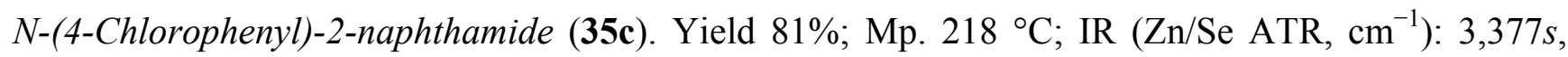
$3,058 w, 1,657 s, 1,628 s, 1,593 s, 1,514 s, 1,492 s, 1,398 s, 1,310 s, 1,097 m, 823 s, 760 s ;{ }^{1} \mathrm{H}-\mathrm{NMR}$ (DMSO- $\left.d_{6}\right)$ [56], $\delta: 10.58(\mathrm{~s}, 1 \mathrm{H}), 8.59(\mathrm{~s}, 1 \mathrm{H}), 8.10-8.08(\mathrm{~m}, 1 \mathrm{H}), 8.08-8.01(\mathrm{~m}, 3 \mathrm{H}), 7.91-7.87(\mathrm{~m}$, 2H), 7.67-7.60 (m, 2H), 7.46-7.42 (m, 2H); ${ }^{13} \mathrm{C}-\mathrm{NMR}$ (DMSO- $\left.d_{6}\right), \delta: 165.54,138.10,134.21,131.95$, 
131.92, 128.78, 128.38, 127.89, 127.88, 127.72, 127.52, 127.20, 126.72, 124.24, 121.78; HR-MS: for $\mathrm{C}_{17} \mathrm{H}_{13} \mathrm{NOCl}[\mathrm{M}+\mathrm{H}]^{+}$calculated $282.0680 \mathrm{~m} / \mathrm{z}$, found $282.0679 \mathrm{~m} / \mathrm{z}$.

N-(2-Bromophenyl)-2-naphthamide (36a) [58]. Yield 70\%; Mp. $123{ }^{\circ} \mathrm{C}$; IR ( $\left.\mathrm{Zn} / \mathrm{Se} \mathrm{ATR}, \mathrm{cm}^{-1}\right)$ : $3,277 \mathrm{~s}, 3,060 w, 1,652 \mathrm{~s}, 1,629 m, 1,578 m, 1,523 s, 1,433 s, 1,304 s, 1,028 m, 777 m, 759 m, 745 s ;{ }^{1} \mathrm{H}-\mathrm{NMR}$ $\left(\mathrm{DMSO}-d_{6}\right), \delta: 10.25(\mathrm{~s}, 1 \mathrm{H}), 8.65(\mathrm{~s}, 1 \mathrm{H}), 8.10-8.08(\mathrm{~m}, 1 \mathrm{H}), 8.07(\mathrm{~s}, 2 \mathrm{H}), 8.03-8.01(\mathrm{~m}, 1 \mathrm{H}), 7.75(\mathrm{dd}$, $J=7.8 \mathrm{~Hz}, J=0.8 \mathrm{~Hz}, 1 \mathrm{H}), 7.68-7.61(\mathrm{~m}, 3 \mathrm{H}), 7.46(\mathrm{td}, J=7.5 \mathrm{~Hz}, J=1.0 \mathrm{~Hz}, 1 \mathrm{H}), 7.25$ (td, $J=7.7 \mathrm{~Hz}, J=1.4 \mathrm{~Hz}, 1 \mathrm{H}) ;{ }^{13} \mathrm{C}-\mathrm{NMR}\left(\mathrm{DMSO}-d_{6}\right), \delta: 165.43,136.70,134.41,132.73,132.13,131.41$, 129.01, 128.93, 128.22, 128.15, 128.15, 127.95, 127.72, 126.94, 124.37, 124.34, 120.68; HR-MS: for $\mathrm{C}_{17} \mathrm{H}_{13} \mathrm{NOBr}[\mathrm{M}+\mathrm{H}]^{+}$calculated $326.0175 \mathrm{~m} / \mathrm{z}$, found $326.0175 \mathrm{~m} / \mathrm{z}$.

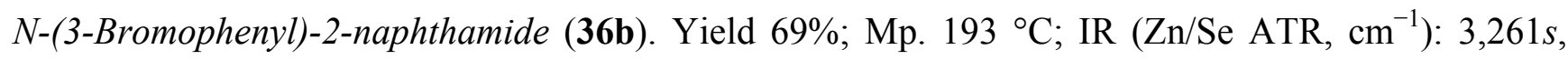
$3,233 s, 3,106 w, 3,056 w, 1,644 s, 1,590 s, 1,526 s, 1,415 s, 1,313 s, 1,300 s, 1,133 m, 778 s, 765 s, 686 m$; ${ }^{1} \mathrm{H}-\mathrm{NMR}\left(\mathrm{DMSO}-d_{6}\right)$ [56], $\delta: 10.59(\mathrm{~s}, 1 \mathrm{H}), 8.59(\mathrm{~s}, 1 \mathrm{H}), 8.18(\mathrm{t}, J=1.9 \mathrm{~Hz}, 1 \mathrm{H}), 8.11-8.08(\mathrm{~m}, 1 \mathrm{H})$, 8.08-8.00 (m, 3H), $7.83(\mathrm{dt}, J=7.7 \mathrm{~Hz}, J=1.6 \mathrm{~Hz}, 1 \mathrm{H}), 7.68-7.61(\mathrm{~m}, 2 \mathrm{H}), 7.37-7.30(\mathrm{~m}, 2 \mathrm{H})$; ${ }^{13} \mathrm{C}-\mathrm{NMR}\left(\mathrm{DMSO}-d_{6}\right.$ ), $\delta: 165.63,140.74,134.23,131.92,131.72,130.43,128.78,127.93,127.90$, $127.75,127.52,126.73,126.08,124.20,122.51,121.25,118.92$; HR-MS: for $\mathrm{C}_{17} \mathrm{H}_{13} \mathrm{NOBr}[\mathrm{M}+\mathrm{H}]^{+}$ calculated $326.0175 \mathrm{~m} / \mathrm{z}$, found $326.0174 \mathrm{~m} / \mathrm{z}$.

$N$-(4-Bromophenyl)-2-naphthamide (36c). Yield 55\%; Mp. $231{ }^{\circ} \mathrm{C}$; IR (Zn/Se ATR, $\left.\mathrm{cm}^{-1}\right): 3,375 s$, $3,283 w, 3,056 w, 1,658 s, 1,591 s, 1,516 s, 1,489 s, 1,395 s, 1,310 s, 1,073 m, 1,009 m, 820 s, 760 s ;{ }^{1} \mathrm{H}-\mathrm{NMR}$ $\left(\mathrm{DMSO}-d_{6}\right)$ [56], $\delta: 10.57(\mathrm{~s}, 1 \mathrm{H}), 8.58(\mathrm{~s}, 1 \mathrm{H}), 8.10-8.08(\mathrm{~m}, 1 \mathrm{H}), 8.08-7.99(\mathrm{~m}, 3 \mathrm{H}), 7.83(\mathrm{~d}$, $J=8.5 \mathrm{~Hz}, 2 \mathrm{H}), 7.67-7.61(\mathrm{~m}, 2 \mathrm{H}) 7.57$ (d, $J=8.5 \mathrm{~Hz}, 2 \mathrm{H}) ;{ }^{13} \mathrm{C}-\mathrm{NMR}$ (DMSO- $d_{6}$ ), $\delta: 165.54,138.52$, $134.21,131.93,131.90,131.30,128.79,127.90,127.72,127.52,126.72,124.24,122.16,122.15$, 115.21; HR-MS: for $\mathrm{C}_{17} \mathrm{H}_{13} \mathrm{NOBr}[\mathrm{M}+\mathrm{H}]^{+}$calculated $326.0175 \mathrm{~m} / \mathrm{z}$, found $326.0173 \mathrm{~m} / \mathrm{z}$.

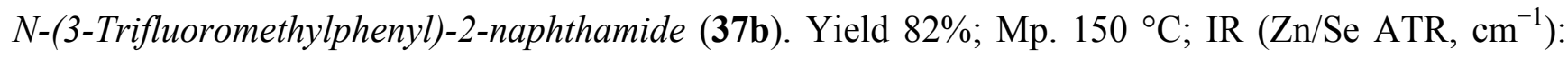
3,261s, 3,100w, 3,062w, 1,648s, 1,602s, 1,552s, 1,445s, 1,434s, 1,324s, 1,309s, 1,165s, 1,107s, 1,069s, $778 m, 697 m$; ${ }^{1} \mathrm{H}-\mathrm{NMR}$ (DMSO-d $\left.d_{6}\right), \delta: 10.75(\mathrm{~s}, 1 \mathrm{H}), 8.63(\mathrm{~s}, 1 \mathrm{H}), 8.32(\mathrm{~s}, 1 \mathrm{H}), 8.15-8.01(\mathrm{~m}, 5 \mathrm{H})$, $7.69-7.60(\mathrm{~m}, 3 \mathrm{H}), 7.47(\mathrm{~d}, J=7.8 \mathrm{~Hz}, 1 \mathrm{H}) ;{ }^{13} \mathrm{C}-\mathrm{NMR}$ (DMSO- $\left.d_{6}\right), \delta: 165.85,139.95,134.29,131.93$, $131.66,129.72,129.31$ (q, $J=32.1 \mathrm{~Hz}$ ), 128.83, 128.02, 127.97, 127.82, 127.55, 126.78, 124.21, $124.06(\mathrm{q}, J=273.1 \mathrm{~Hz}), 123.68,119.79(\mathrm{q}, J=3.8 \mathrm{~Hz}), 116.31$ (q, $J=3.8 \mathrm{~Hz})$; HR-MS: for $\mathrm{C}_{18} \mathrm{H}_{13} \mathrm{NOF}_{3}[\mathrm{M}+\mathrm{H}]^{+}$calculated $316.0944 \mathrm{~m} / \mathrm{z}$, found $316.0942 \mathrm{~m} / z$.

$N$-(4-Trifluoromethylphenyl)-2-naphthamide (37c). Yield 80\%; Mp. $224{ }^{\circ} \mathrm{C}$; IR (Zn/Se ATR, $\left.\mathrm{cm}^{-1}\right)$ : $3,372 s, 3,073 w, 1,662 s, 1,600 s, 1,556 s, 1,514 s, 1,408 s, 1,316 s, 1,159 s, 1,112 s, 1,067 s, 1,018 m, 836 s$, 826s, 764m; ${ }^{1} \mathrm{H}-\mathrm{NMR}$ (DMSO- $\left.d_{6}\right), \delta: 10.78(\mathrm{~s}, 1 \mathrm{H}), 8.62(\mathrm{~s}, 1 \mathrm{H}), 8.12-8.01(\mathrm{~m}, 6 \mathrm{H}), 7.76(\mathrm{~d}$, $J=8.8 \mathrm{~Hz}, 2 \mathrm{H}), 7.69-7.61(\mathrm{~m}, 2 \mathrm{H}) ;{ }^{13} \mathrm{C}-\mathrm{NMR}$ (DMSO- $\left.d_{6}\right), \delta: 165.97,142.80,134.32,131.93,131.71$, 128.87, 128.13, 127.97, 127.87, 127.58, 126.81, 125.80 (q, $J=3.8 \mathrm{~Hz}$ ), 124.29, 124.29 (q, $J=270.9 \mathrm{~Hz}$ ), $123.57\left(\mathrm{q}, J=32.1 \mathrm{~Hz}\right.$ ), 120.07; HR-MS: for $\mathrm{C}_{18} \mathrm{H}_{13} \mathrm{NOF}_{3}[\mathrm{M}+\mathrm{H}]^{+}$calculated $316.0944 \mathrm{~m} / z$, found $316.0942 \mathrm{~m} / \mathrm{z}$. 
N-(2-Nitrophenyl)-2-naphthamide (38a). Yield 66\%; Mp. $136{ }^{\circ} \mathrm{C}$ (Mp. 138-139 ${ }^{\circ} \mathrm{C}$ [59]); IR (Zn/Se ATR, $\left.\mathrm{cm}^{-1}\right): 3,383 s, 3,124 w, 3,054 w, 1,678 s, 1,582 s, 1,493 s, 1,448 s, 1,427 s, 1,335 s, 1,284 s, 1,273 s$, $1,195 s, 759 s, 738 s ;{ }^{1} \mathrm{H}-\mathrm{NMR}$ (DMSO- $\left.d_{6}\right), \delta: 10.97(\mathrm{~s}, 1 \mathrm{H}), 8.62(\mathrm{~s}, 1 \mathrm{H}), 8.15-8.00(\mathrm{~m}, 5 \mathrm{H}), 7.86-7.82$ $(\mathrm{m}, 1 \mathrm{H}), 7.81-7.76(\mathrm{~m}, 1 \mathrm{H}), 7.70-7.62(\mathrm{~m}, 2 \mathrm{H}), 7.44(\mathrm{t}, J=7.7 \mathrm{~Hz}, 1 \mathrm{H}) ;{ }^{13} \mathrm{C}-\mathrm{NMR}$ (DMSO- $\left.d_{6}\right)$, $\delta: 165.32,142.68,134.45,133.94,131.98,131.63,130.83,128.92,128.28,128.20,128.04,127.60$, 126.91, 125.80, 125.43, 124.88, 123.92; HR-MS: for $\mathrm{C}_{17} \mathrm{H}_{13} \mathrm{~N}_{2} \mathrm{O}_{3}[\mathrm{M}+\mathrm{H}]^{+}$calculated $293.0921 \mathrm{~m} / z$, found $293.0919 \mathrm{~m} / \mathrm{z}$.

N-(3-Nitrophenyl)-2-naphthamide (38b). Yield 68\%; Mp. $177{ }^{\circ} \mathrm{C}$; IR (Zn/Se ATR, $\left.\mathrm{cm}^{-1}\right): 3,269 s$, $3,093 w, 1,648 s, 1,626 s, 1,522 s, 1,429 s, 1,341 s, 1,321 s, 1,288 s, 1,272 s, 761 s, 736 s, 669 s ;{ }^{1} \mathrm{H}-\mathrm{NMR}$ (DMSO- $d_{6}$ ) [56], $\delta: 10.88(\mathrm{~s}, 1 \mathrm{H}), 8.85(\mathrm{t}, J=2.1 \mathrm{~Hz}, 1 \mathrm{H}), 8.63(\mathrm{~s}, 1 \mathrm{H}), 8.26$ (ddd, $J=8.2 \mathrm{~Hz}, J=2.0 \mathrm{~Hz}$, $J=0.9 \mathrm{~Hz}, 1 \mathrm{H}), 8.11-7.99(\mathrm{~m}, 4 \mathrm{H}), 7.97(\mathrm{ddd}, J=8.2 \mathrm{~Hz}, J=2.0 \mathrm{~Hz}, J=0.9 \mathrm{~Hz}, 1 \mathrm{H}), 7.69-7.61$ (m, $3 \mathrm{H}$ ); ${ }^{13} \mathrm{C}-\mathrm{NMR}$ (DMSO-d $d_{6}$ ), $\delta: 165.94,147.88,140.34,134.35,131.92,131.42,129.92,128.85$, 128.14, 128.02, 127.91, 127.58, 126.82, 126.06, 124.20, 117.96, 114.30; HR-MS: for $\mathrm{C}_{17} \mathrm{H}_{13} \mathrm{~N}_{2} \mathrm{O}_{3}$ $[\mathrm{M}+\mathrm{H}]^{+}$calculated $293.0921 \mathrm{~m} / \mathrm{z}$, found $293.0917 \mathrm{~m} / \mathrm{z}$.

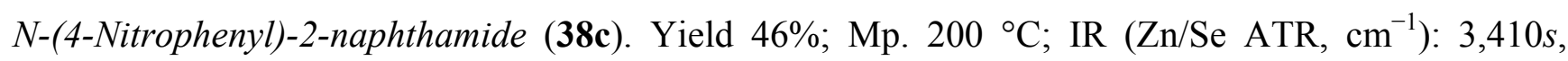
$3,054 w, 1,679 s, 1,611 s, 1,595 s, 1,538 s, 1,499 s, 1,481 s, 1,324 s, 1,302 s, 1,284 s, 1,243 s, 1,109 s, 849 s$, $771 s, 750 s ;{ }^{1} \mathrm{H}-\mathrm{NMR}\left(\mathrm{DMSO}-d_{6}\right)$ [56], $\delta: 10.99(\mathrm{~s}, 1 \mathrm{H}), 8.62(\mathrm{~s}, 1 \mathrm{H}), 8.29$ (dd, $J=9.3 \mathrm{~Hz}, J=2.3 \mathrm{~Hz}$, $2 \mathrm{H}), 8.13-8.01(\mathrm{~m}, 6 \mathrm{H}), 7.69-7.61(\mathrm{~m}, 2 \mathrm{H}) ;{ }^{13} \mathrm{C}-\mathrm{NMR}$ (DMSO- $\left.d_{6}\right), \delta: 166.20,145.45,142.43,134.41$, 131.90, 131.43, 128.92, 128.37, 128.07, 128.02, 127.61, 126.88, 124.67, 124.27, 119.80; HR-MS: for $\mathrm{C}_{17} \mathrm{H}_{13} \mathrm{~N}_{2} \mathrm{O}_{3}[\mathrm{M}+\mathrm{H}]^{+}$calculated $293.0921 \mathrm{~m} / \mathrm{z}$, found $293.0918 \mathrm{~m} / \mathrm{z}$.

\subsection{Study of Inhibition Photosynthetic Electron Transport (PET) in Spinach Chloroplasts}

Chloroplasts were prepared from spinach (Spinacia oleracea L.) according to Masarovicova and Kralova [60]. The inhibition of photosynthetic electron transport (PET) in spinach chloroplasts was determined spectrophotometrically (Genesys 6, Thermo Scientific, USA), using an artificial electron acceptor 2,6-dichlorophenol-indophenol (DCIPP) according to Kralova et al. [61], and the rate of photosynthetic electron transport was monitored as a photoreduction of DCPIP. The measurements were carried out in phosphate buffer $(0.02 \mathrm{~mol} / \mathrm{L}, \mathrm{pH} 7.2)$ containing sucrose $(0.4 \mathrm{~mol} / \mathrm{L})$, $\mathrm{MgCl}_{2}(0.005 \mathrm{~mol} / \mathrm{L})$ and $\mathrm{NaCl}(0.015 \mathrm{~mol} / \mathrm{L})$. The chlorophyll content was $30 \mathrm{mg} / \mathrm{L}$ in these experiments and the samples were irradiated $\left(\sim 100 \mathrm{~W} / \mathrm{m}^{2}\right.$ with $10 \mathrm{~cm}$ distance) with a halogen lamp $(250 \mathrm{~W})$ using a $4 \mathrm{~cm}$ water filter to prevent warming of the samples (suspension temperature $22^{\circ} \mathrm{C}$ ). The studied compounds were dissolved in DMSO due to their limited water solubility. The applied DMSO concentration (up to 4\%) did not affect the photochemical activity in spinach chloroplasts. The inhibitory efficiency of the studied compounds was expressed by $\mathrm{IC}_{50}$ values, i.e., by molar concentration of the compounds causing $50 \%$ decrease in the oxygen evolution rate relative to the untreated control. The comparable $\mathrm{IC}_{50}$ value for a selective herbicide 3-(3,4-dichlorophenyl)-1,1-dimethylurea, DCMU (Diurone $^{\circledR}$ ) was about $1.9 \mu \mathrm{mol} / \mathrm{L}$. The results are summarized in Tables 1 and 2. 


\subsection{In Vitro Antimycobacterial Evaluation}

Clinical isolates of Mycobacterium tuberculosis CUH071 (Cork University Hospital TB lab, with partial INH and PZA resistance), M. avium complex CIT19/06, M. avium paratuberculosis ATCC19698, and M. kansasii CIT11/06 were grown in Middlebrook broth (MB), supplemented with

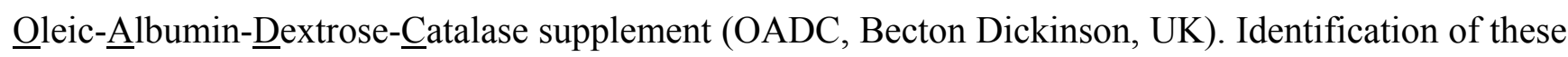
isolates was performed using biochemical and molecular protocols. At log phase growth, culture $(10 \mathrm{~mL})$ was centrifuged at 15,000 rpm/20 min using a bench top centrifuge (Model CR 4-12, Jouan Inc., UK). Following removal of the supernatant, the pellet was washed in fresh Middlebrook 7H9GC broth and re-suspended in fresh supplemented $\mathrm{MB}(10 \mathrm{~mL})$. The turbidity was adjusted to match McFarland standard No. $1\left(3 \times 10^{8} \mathrm{cfu}\right)$ with MB broth. A further 1:20 dilution of the culture was then performed in MB broth.

The antimicrobial susceptibility of all four mycobacterial species was investigated in a 96-well plate format. In these experiments, sterile deionised water $(300 \mu \mathrm{L})$ was added to all outer-perimeter wells of the plates to minimize evaporation of the medium in the test wells during incubation. Each evaluated compound $(100 \mu \mathrm{L})$ was incubated with each of the mycobacterial species $(100 \mu \mathrm{L})$. Dilutions of each compound were prepared in duplicate. For all synthesized compounds, final concentrations ranged from $1,000 \mu \mathrm{g} / \mathrm{mL}$ to $8 \mu \mathrm{g} / \mathrm{mL}$. All compounds were prepared in DMSO and subsequent dilutions were made in supplemented MB. The plates were sealed with parafilm and incubated at $37{ }^{\circ} \mathrm{C}$, for 5 days in the case of M. kansasii and M. avium complex, 7 days in the case of M. tuberculosis and 11 days in the case of M. avium paratuberculosis. Following incubation, a 10\% addition of alamarBlue (AbD Serotec) was mixed into each well and readings at $570 \mathrm{~nm}$ and $600 \mathrm{~nm}$ were taken, initially for background subtraction and subsequently after $24 \mathrm{~h}$ re-incubation. The background subtraction is necessary for strongly coloured compounds, where the colour may interfere with the interpretation of any colour change. For non-interfering compounds, a blue colour in the well was interpreted as an absence of growth and a pink colour was scored as growth. The MIC was initially defined as the lowest concentration which prevented a visual colour change from blue to pink. Isoniazid (INH) and pyrazinamide (PZA) were used as the standards as it is a clinically used antimycobacterial drug. The MIC for mycobacteria was defined as a $90 \%$ or greater $\left(\mathrm{IC}_{90}\right)$ reduction of growth in comparison with the control. The $\mathrm{MIC} / \mathrm{IC}_{90}$ value is routinely and widely used in bacterial assays and is a standard detection limit according to the Clinical and Laboratory Standards Institute (CLSI, www.clsi.org). The results are summarized in Table 3.

\subsection{In Vitro Cytotoxicity Assay}

Human monocytic leukemia THP-1 cells were obtained from the European Collection of Cell Cultures (ECACC). These cells were routinely cultured in RPMI medium supplemented with 10\% fetal bovine serum, $2 \%$ L-glutamine, $1 \%$ penicillin and streptomycin at $37{ }^{\circ} \mathrm{C}$ with $5 \% \mathrm{CO}_{2}$. The tested compounds were dissolved in DMSO and added in four increasing concentrations to the cell suspension in the culture medium. Subsequently, the cells were incubated for $24 \mathrm{~h}$ at $37{ }^{\circ} \mathrm{C}$ with $5 \%$ $\mathrm{CO}_{2}$. Cell toxicity was determined using a WST-1 assay kit (Roche Diagnostics, Mannheim, Germany) according to the manufacturer's instructions. For WST-1 assays, cells were seeded into 96-well plates $\left(5 \times 10^{4}\right.$ cells $\cdot$ well $^{-1}$ in $100 \mu \mathrm{L}$ culture medium) in triplicate in serum-free RPMI 1640 medium, and 
measurements were taken $24 \mathrm{~h}$ after the treatment with tested compounds. The median lethal dose values, $\mathrm{LD}_{50}$, were deduced through the production of a dose-response curve. All data were evaluated using GraphPad Prism 5.00 software (GraphPad Software, San Diego, CA, USA, http://www.graphpad.com). The results are summarized in Table 3.

\section{Conclusions}

A series of thirty-five substituted quinoline-2-carboxamides and thirty-three substituted naphthalene-2-carboxamides were prepared and characterized. The prepared compounds were tested for their ability to inhibit photosynthetic electron transport (PET) in spinach chloroplasts (Spinacia oleracea L.) and for their antituberculosis/antimycobacterial activity. Two compounds, $N$-benzyl-2naphthamide (29) and $N$-(2-hydroxyphenyl)quinoline-2-carboxamide (12a) showed relatively high PET inhibition. $N$-(2-Phenylethyl)quinoline-2-carboxamide (11), $N$-cycloheptylquinoline-2-carboxamide (7) and $\mathrm{N}$-cyclohexylquinoline-2-carboxamide (6) expressed high activity against Mycobacterium tuberculosis. 1-(2-Naphthoyl)pyrrolidine (22) and 2-(pyrrolidin-1-ylcarbonyl)quinoline (3) showed high activity against M. kansasii and M. avium paratuberculosis. All five compounds exhibited activity comparable with or higher than the standards isoniazid or pyrazinamide. Lipophilicity was fundamental for the biological activities of all compounds in both biological assays. It can be stated that the dependence of PET-inhibiting activity on the lipophilicity decreases with increasing lipophilicity, while antimycobacterial activity increases with lipophilicity increase. Highly effective compounds against $M$. tuberculosis were detected, namely quinaldinamides, while naphtamides were more active against the other mycobacterial species. Substituted $N$-quinaldinanilides and/or $N$-naphtanilides seem to be less effective than other discussed $N$-nonaromatic amide derivatives. The most effective antimycobacterial compounds 11, 7, 3 and 22 were tested for their in vitro cytotoxicity against THP-1 cells. According to the calculated selectivity index of compounds 11, 3 and $\mathbf{2 2}$ it can be concluded that the discussed amides can be considered as promising agents for subsequent design of novel antitubercular/ antimycobacterial agents.

\section{Acknowledgements}

This study was supported by the IGA VFU Brno 49/2011/FaF, the Slovak Grant Agency VEGA, Grant No. 1/0612/11, by Sanofi-Aventis Pharma Slovakia and by the Irish Department of Agriculture Fisheries and Food 08RDCIT601.

\section{References and Notes}

1. Roth, H.J.; Fenner, H. Arzneistoffe, 3rd, ed.; Deutscher Apotheker Verlag: Stuttgart, Germany, 2000; pp. 51-114.

2. Good, N.E. Inhibitors of the Hill reaction. Plant Physiol. 1961, 36, 788-803.

3. Dolezal, M.; Palek, L.; Vinsova, J.; Buchta, V.; Jampilek, J.; Kralova, K. Substituted pyrazinecarboxamides: Synthesis and biological evaluation. Molecules 2006, 11, 242-256. 
4. Dolezal, M.; Cmedlova, P.; Palek, L.; Vinsova, J.; Kunes, J.; Buchta, V.; Jampilek, J.; Kralova, K. Synthesis and antimycobacterial evaluation of substituted pyrazinecarboxamides. Eur. J. Med. Chem. 2008, 43, 1105-1113.

5. Dolezal, M.; Zitko, J.; Osicka, Z.; Kunes, J.; Vejsova, M.; Buchta, V.; Dohnal, J.; Jampilek, J.; Kralova, K. Synthesis, antimycobacterial, antifungal and photosynthesis-inhibiting activity of chlorinated N-phenylpyrazine-2-carboxamides. Molecules 2010, 15, 8567-8581.

6. Otevrel, J.; Mandelova, Z.; Pesko, M.; Guo, J.; Kralova, K.; Sersen, F.; Vejsova, M.; Kalinowski, D.; Kovacevic, Z.; Coffey, A.; Csollei, J.; et al. Investigating the spectrum of biological activity of ring-substituted salicylanilides and carbamoylphenylcarbamates. Molecules 2010, 15, 8122-8142.

7. Dolezal, M.; Kralova, K. Synthesis and evaluation of pyrazine derivatives with herbicidal activity. In Herbicides, Theory and Applications; Soloneski, S., Larramendy, M.L., Eds.; InTech: Rijeka, Croatia, 2011; pp. 581-610.

8. Imramovsky, A.; Pesko, M.; Kralova, K.; Vejsova, M.; Stolarikova, J.; Vinsova, J.; Jampilek, J. Investigating spectrum of biological activity of 4- and 5-chloro-2-hydroxy-N-[2-(arylamino)-1alkyl-2-oxoethyl]benzamides. Molecules 2011, 16, 2414-2430.

9. Imramovsky, A.; Pesko, M.; Monreal-Ferriz, J.; Kralova, K.; Vinsova, J.; Jampilek, J. Photosynthesis-inhibiting efficiency of 4-chloro-2-(chlorophenylcarbamoyl)phenyl alkyl-carbamates. Bioorg. Med. Chem. Lett. 2011, 21, 4564-4567.

10. Harris, C.R.; Thorarensen, A. Advances in the discovery of novel antibacterial agents during the year 2002. Curr. Med. Chem. 2004, 11, 2213-2243.

11. Andries, K.; Verhasselt, P.; Guillemont, J.; Gohlmann, H.W.; Neefs, J.M.; Winkler, H.; van Gestel, J.; Timmerman, P.; Zhu, M.; Lee, E.; et al. A diarylquinoline drug active on the ATP synthase of Mycobacterium tuberculosis. Science 2005, 307, 223-227.

12. Vangapandu, S.; Jain, M.; Jain, R.; Kaur, S.; Singh, P.P. Ring-substituted quinolines as potential anti-tuberculosis agents. Bioorg. Med. Chem. 2004, 12, 2501-2508.

13. Jampilek, J.; Dolezal, M.; Kunes, J.; Buchta, V.; Kralova, K. Quinaldine derivatives: Preparation and biological activity. Med. Chem. 2005, 1, 591-599.

14. Musiol, R.; Jampilek, J.; Buchta, V.; Niedbala, H.; Podeszwa, B.; Palka, A.; Majerz-Maniecka, K.; Oleksyn, B.; Polanski, J. Antifungal properties of new series of quinoline derivatives. Bioorg. Med. Chem. 2006, 14, 3592-3598.

15. Jampilek, J.; Musiol, R.; Pesko, M.; Kralova, K.; Vejsova, M.; Carroll, J.; Coffey, A.; Finster, J.; Tabak, D.; Niedbala, H.; et al. Ring-substituted 4-hydroxy-1H-quinolin-2-ones: Preparation and biological activity. Molecules 2009, 14, 1145-1159.

16. Jampilek, J.; Musiol, R.; Finster, J.; Pesko, M.; Carroll, J.; Kralova, K.; Vejsova, M.; O’Mahony, J.; Coffey, A.; Dohnal, J.; et al. Investigating biological activity spectrum for novel styrylquinazoline analogues. Molecules 2009, 14, 4246-4265.

17. Musiol, R.; Jampilek, J.; Nycz, J.E.; Pesko, M.; Carroll, J.; Kralova, K.; Vejsova, M.; O’Mahony, J.; Coffey, A.; Mrozek, A.; et al. Investigating the activity spectrum for ring-substituted 8-hydroxyquinolines. Molecules 2010, 15, 288-304.

18. Musiol, R.; Jampilek, J.; Kralova, K.; Richardson, D.R.; Kalinowski, D.; Podeszwa, B.; Finster, J.; Niedbala, H.; Palka, A.; Polanski, J. Investigating biological activity spectrum for novel quinoline analogues. Bioorg. Med. Chem. 2007, 15, 1280-1288. 
19. Podeszwa, B.; Niedbala, H.; Polanski, J.; Musiol, R.; Tabak, D.; Finster, J.; Serafin, K.; Wietrzyk, J.; Boryczka, S.; Mol, W.; et al. Investigating the antiproliferative activity of quinoline-5,8-dione analogues on tumour cell lines. Bioorg. Med. Chem. Lett. 2007, 17, 6138-6141.

20. Mrozek-Wilczkiewicz, A.; Kalinowski, D.; Musiol, R.; Finster, J.; Szurko, A.; Serafin, K.; Knas, M.; Kamalapuram, S.K.; Kovacevic, Z.; Jampilek, J.; et al. Investigating anti-proliferative activity of styrylazanaphthalenes and azanaphthalenediones. Bioorg. Med. Chem. 2010, 18, 2664-2671.

21. Semar, M.; Anke, H.; Arendholz, W.R.; Velten, R.; Steglich, W. Lachnellins A, B, C, D, and naphthalene-1,3,8-triol, biologically active compounds from a Lachnellula species (Ascomycetes). Z. Naturforsch C 1996, 51, 500-512.

22. Ulubelen, A.; Topcu, G.; Johansson, C.B. Norditerpenoids and diterpenoids from Salvia multicaulis with antituberculous activity. J. Nat. Prod. 1997, 60, 1275-1280.

23. Ezra, D.; Hess, W.M.; Strobel, G.A. New endophytic isolates of Muscodor albus, a volatile-antibiotic-producing fungus. Microbiology 2004, 150, 4023-4031.

24. Huang, W.; Li, J.; Zhang, W.; Zhou, Y.; Xie, C.; Luo, Y.; Li, Y.; Wang, J.; Li, J.; Lu, W. Synthesis of miltirone analogues as inhibitors of Cdc25 phosphatases. Bioorg. Med. Chem. Lett. 2006, 16, 1905-1908.

25. Hedstrom, L.K.; Striepen, B. (Brandeis University \& University of Georfia Research Foundation). Compounds and methods for treating mammalian gastrointestinal parasitic infections. US Patent US2010/0022547 A1, 28 January 2010.

26. Musiol, R.; Tabak, D.; Niedbala, H.; Podeszwa, B.; Jampilek, J.; Kralova, K.; Dohnal, J.; Finster, J.; Mencel, A.; Polanski, J. Investigating biological activity spectrum for novel quinoline analogues 2: Hydroxyquinolinecarboxamides with photosynthesis inhibiting activity. Bioorg. Med. Chem. 2008, 16, 4490-4499.

27. Black, C.C. Photosynthetic phosphorylation and associated reactions in the presence of a new group of uncouplers: Salicylanilides. Biochim. Biophys. Acta 1968, 162, 294-296.

28. Draber, W.; Tietjen, K.; Kluth, J.F.; Trebst, A. Herbicides in photosynthesis research. Angew. Chem. Int. Ed. Engl. 1991, 3, 1621-1633.

29. Tischer, W.; Strotmann, H. Relationship between inhibitor binding by chloroplasts and inhibition of photosynthetic electron-transport. Biochim. Biophys. Acta 1977, 460, 113-125.

30. Trebst, A.; Draber, W. Structure activity correlations of recent herbicides in photosynthetic reactions. In Advances in Pesticide Science; Greissbuehler, H., Ed.; Pergamon Press: Oxford, UK, 1979; pp. 223-234.

31. Bowyer, J.R.; Camilleri, P.; Vermaas, W.F.J. Photosystem II and its interaction with herbicides. In Herbicides, Topics in Photosynthesis; Baker, N.R., Percival, M.P., Eds.; Elsevier: Amsterdam, The Netherlands, 1991; Volume 10, pp. 27-85.

32. Shaner, D.L. Herbicide safety relative to common targets in plants and mammals. Pest Manag. Sci. 2004, 60, 17-24.

33. Delaney, J.; Clarke, E.; Hughes, D.; Rice, M. Modern agrochemical research: A missed opportunity for drug discovery? Drug Discov. Today 2006, 11, 839-845.

34. Duke, S.O. Herbicide and pharmaceutical relationships. Weed Sci. 2010, 58, 334-339.

35. Norrington, F.E.; Hyde, R.M.; Williams, S.G.; Wotton, R. Physicochemical-activity relations in practice. 1. Rational and self-consistent data bank. J. Med. Chem. 1975, 18, 604-607. 
36. Taft, R.W., Jr. Separation of polar, steric, and resonance effects in reactivity. In Steric Effect in Organic Chemistry; Newman, M.S., Ed.; John Wiley \& Sons: New York, NY, USA, 1956; Chapter 13.

37. Takahata, Y.; Chong, D.P. Estimation of Hammett sigma constants of substituted benzenes through accurate density-functional calculation of core-electron binding energy shifts. Int. J. Quantum Chem. 2005, 103, 509-515.

38. Chapman, N.B.; Shorter, J. Advances in Linear Free Energy Relationships; Plenum Press: London, UK, 1972.

39. Wang, X.; Wang, D.Z. Aerobic oxidation of secondary benzylic alcohols and direct oxidative amidation of aryl aldehydes promoted by sodium hydride. Tetrahedron 2011, 67, 3406-3411.

40. Coppa, F.; Fontana, F.; Lazzarini, E.; Minisci, F. A facile, convenient and selective homolytic carbamoylation of heteroaromatic bases. Heterocycles 1993, 36, 2687-2696.

41. Kampen, G.C.T.; Andersen, H.S. (Novo Nordisk A/S). Combination therapy using an $11 \beta$-hydroxysteroid dehydrogenase type 1 inhibitor and a glucocorticoid receptor agonist to minimize the side effects associated with glucocorticoid receptor agonist therapy. PCT Int. Appl. WO 2004089415 A2, 21 October 2004.

42. Davis, J.W. Studies with quinolines. I. Synthesis of quinaldic acid and some of its amide derivatives. J. Org. Chem. 1959, 24, 1691-1694.

43. Ren, W.; Yamane, M. Mo(CO) 6 -Mediated carbamoylation of aryl halides. J. Org. Chem. 2010, $75,8410-8415$.

44. Gracheva, I.N.; Ioffina, D.I.; Tochilkin, A.I.; Gorkin, V.Z. Monoamine oxidase inhibitors based on 2-, 4-, and 8-substituted quinolones. Pharm. Chem. J. 1991, 25, 160-165.

45. Dzadzic, P.M.; Bastic, B.L.; Piletic, M.V. Reaction between 2-quinolinecarboxylic acid and some aromatic and heterocyclic amines. Glas. Hem. Drus. Beograd 1971, 36, 137-142.

46. Petrie, C.; Orme, M.W.; Baindur, N.; Robbins, K.G.; Harris, S.M.; Kontoyianni, M.; Hurley, L.H.; Kerwin, S.M.; Mundy, G.R. (Zymogenetics, Inc. \& Osteoscreen, Inc. \& University of Texas). Compositions and Methods for Treating Bone Deficit Conditions. WO Patent WO 1997015308, 1 May 1997.

47. Chan, L.; Jin, H.; Stefanac, T.; Wang, W.; Lavallee, J.F.; Bedard, J.; May, S. Isoquinoline-6carboxamides as potent and selective anti-human cytomegalovirus (HCMV) inhibitors. Bioorg. Med. Chem. Lett. 1999, 9, 2583-2586.

48. Schaefer, W.; Neubert, P. Massenspektren heterocyclischer carbonsaureamide-I: Pyridin- und chinolincarbonsaureanilide. Tetrahedron 1969, 25, 315-327.

49. Kiselyov, A.S. Reaction of N-fluoropyridinium fluoride with isonitriles and $\mathrm{TMSN}_{3}$ : A convenient one-pot synthesis of tetrazol-5-yl pyridines. Tetrahedron Lett. 2005, 46, 4851-4854.

50. Vieth, P. Zur Kenntnis der $\beta$-Naphtoesaure. Chem. Ber. 1875, 8, 1278-1281.

51. Rahman, O.; Kihlberg, T.; Langstrom, B. Aryl triflates and $\left[{ }^{11} \mathrm{C}\right] /\left({ }^{13} \mathrm{C}\right)$ carbon monoxide in the synthesis of ${ }^{11} \mathrm{C}$ - $/{ }^{13} \mathrm{C}$-amides. J. Org. Chem. 2003, 68, 3558-3562.

52. Berndt, D.C.; Faburada A.L. Reaction of acyl azide and amines. Kinetics and mechanism. J. Org. Chem. 1982, 47, 4167-4169.

53. Lauwagie, S.; Millet, R.; Pommery, J.; Depreux, P.; Henichart, J.P. Expeditious synthesis of 2-aryl-substituted imidazolines and imidazoles. Heterocycles 2006, 68, 1149-1162. 
54. Hofmann, A.W. Uber die Menaphtoxylsaure und ihre Abkommlinge. Chem. Ber. 1868, 1, 38-43.

55. Zhang, X.; Guo, L.; Wu, F.Y.; Jiang, Y.B. Development of fluorescent sensing of anions under excited-state intermolecular proton transfer signaling mechanism. Org. Lett. 2003, 5, 2667-2670.

56. Liu, L.H.; Guo, L.; Liu, C.H.; Zhang, X.; Jiang, Y.B. Intramolecular charge transfer with 1-naphthanilides and 2-naphthanilides. Chin. J. Chem. 2005, 23, 857-864.

57. Kushner, S.; Cassell, R.I.; Morton, J.; Williams J.H. Anticonvulsants. N-Benzylamides. J. Org. Chem. 1951, 16, 1283-1288.

58. Barbero, N.; Carril, M.; San Martin, R.; Dominguez, S. Copper-catalysed intramolecular O-arylation of aryl chlorides and bromides: A straightforward approach to benzo[d]oxazoles in water. Tetrahedron 2007, 63, 10425-10432.

59. Abramovitch, R.A.; Hey, D.H.; Long, R.A. Internuclear cyclisation. Part XII. The synthesis of some benzophenanthridones. Abnormal reaction of 1-amino- $N$-methyl-2-naphthanilide. J. Chem. Soc. 1957, 1781-1788.

60. Masarovicova, E.; Kralova, K. Approaches to measuring plant photosynthesis activity. In Handbook of Photosynthesis, 2nd ed.; Pessarakli, M., Ed.; Taylor \& Francis Group: Boca Raton, FL, USA, 2005; pp. 617-656.

61. Kralova, K.; Sersen, F.; Sidoova, E. Photosynthesis inhibition produced by 2-alkylthio-6-Rbenzothiazoles. Chem. Pap. 1992, 46, 348-350.

Sample Availability: Samples of the compounds are available from the authors.

(C) 2012 by the authors; licensee MDPI, Basel, Switzerland. This article is an open access article distributed under the terms and conditions of the Creative Commons Attribution license (http://creativecommons.org/licenses/by/3.0/). 